\title{
Relaxation for an optimal design problem with linear growth and perimeter penalization
}

\author{
Graça Carita*, Elvira Zappale ${ }^{\dagger}$
}

July 2,2018

\begin{abstract}
The paper is devoted to the relaxation and integral representation in the space of functions of bounded variation for an integral energy arising from optimal design problems. The presence of a perimeter penalization is also considered in order to avoid non existence of admissible solutions, besides this leads to an interaction in the limit energy. Also more general models have been taken into account.
\end{abstract}

Keywords: Relaxation, functions of bounded variation, perimeter penalization.

MSC2010 classification: 49J45, 26B30.

\section{Introduction}

The optimal design problem, devoted to find the minimal energy configurations of a mixture of two conductive materials, has been widely studied since the pioneering papers [28, 29, 30]. It is well known that, given a container $\Omega$ and prescribing only the volume fraction of the material where it is expected to have a certain conductivity, an optimal configuration might not exist. To overcome this difficulty, Ambrosio and Buttazzo in [6] imposed a perimeter penalization and studied the following minimization problem

$$
\min \left\{\int_{E}\left(\alpha|D u|^{2}+g_{1}(x, u)\right) d x+\int_{\Omega \backslash E}\left(\beta|D u|^{2}+g_{2}(x, u)\right) d x+\sigma P(E, \Omega): E \subset \Omega, u \in H_{0}^{1}(\Omega)\right\},
$$

finding the solution $(u, E)$ and describing the regularity properties of the optimal set $E$.

In this paper we are considering the minimization of a similar functional, where the energy density $|\cdot|^{2}$ has been replaced by more general $W_{i}, i=1,2$ without any convexity assumptions and with linear growth, and since the lower order terms $g_{1}(x, u)$ and $g_{2}(x, u)$ do not play any role in the asymptotics, we omit them in our subsequent analysis. The case of $W_{i}, i=1,2$, not convex with superlinear growth has been studied in the context of thin films in [16].

Thus, given $\Omega$ a bounded open subset of $\mathbb{R}^{N}$, we assume that $W_{i}: \mathbb{R}^{d \times N} \rightarrow \mathbb{R}$ are continuous functions such that there exist positive constants $\alpha, \beta$ for which

$$
\alpha|\xi| \leq W_{i}(\xi) \leq \beta(1+|\xi|) \text { for every } \xi \in \mathbb{R}^{d \times N}, \quad i=1,2 .
$$

We consider the following optimal design problem

$$
\inf _{\substack{u \in W^{1,1}\left(\Omega ; \mathbb{R}^{d}\right) \\ \chi_{E} \in B V(\Omega ;\{0,1\})}}\left\{\int_{\Omega}\left(\chi_{E} W_{1}(\nabla u)+\left(1-\chi_{E}\right) W_{2}\right)(\nabla u) d x+P(E ; \Omega): u=u_{0} \text { on } \partial \Omega\right\}
$$

*CIMA-UE, Departamento de Matemática, Universidade de Évora, Rua Romão Ramalho, 59 7000-671 Évora, Portugal. E-mail: gcarita@uevora.pt

†D.I.In., Università degli Studi di Salerno, Via Giovanni Paolo II, 132, 84084 Fisciano (SA) Italy. E-mail: ezappale@unisa.it 
where $\chi_{E}$ is the characteristic function of $E \subset \Omega$ which has finite perimeter, see (2.2) below.

Note that by (2.2) and the definition of total variation, $P(E ; \Omega)=\left|D \chi_{E}\right|(\Omega)$ and we are lead to the subsequent minimum problem

$$
\inf _{\substack{u \in W^{1,1}\left(\Omega ; \mathbb{R}^{d}\right) \\ \chi_{E} \in B V(\Omega ;\{0,1\})}}\left\{\int_{\Omega}\left(\chi_{E} W_{1}+\left(1-\chi_{E}\right) W_{2}\right)(\nabla u) d x+\left|D \chi_{E}\right|(\Omega): u=u_{0} \text { on } \partial \Omega\right\} .
$$

The lack of convexity of the energy requires a relaxation procedure. To this end we start by localizing our energy, first we introduce the functional $F_{\mathcal{O D}}: L^{1}(\Omega ;\{0,1\}) \times L^{1}\left(\Omega ; \mathbb{R}^{d}\right) \times \mathcal{A}(\Omega) \rightarrow[0,+\infty]$ defined by

$F_{\mathcal{O D}}(\chi, u ; A):= \begin{cases}\int_{A}\left(\chi_{E} W_{1}(\nabla u)+\left(1-\chi_{E}\right) W_{2}(\nabla u)\right) d x+\left|D \chi_{E}\right|(A) & \text { in } B V(A ;\{0,1\}) \times W^{1,1}\left(A ; \mathbb{R}^{d}\right) \\ +\infty & \text { otherwise. }\end{cases}$

Then we consider the relaxed localized energy of (1.3) given by

$$
\begin{array}{r}
\mathcal{F}_{\mathcal{O D}}(\chi, u ; A):=\inf \left\{\liminf _{n \rightarrow \infty} \int_{A}\left(\chi_{n} W_{1}\left(\nabla u_{n}\right)+\left(1-\chi_{n}\right) W_{2}\left(\nabla u_{n}\right)\right) d x+\left|D \chi_{n}\right|(A):\left\{u_{n}\right\} \subset W^{1,1}\left(A ; \mathbb{R}^{d}\right),\right. \\
\left.\left\{\chi_{n}\right\} \subset B V(A ;\{0,1\}), u_{n} \rightarrow u \text { in } L^{1}\left(A ; \mathbb{R}^{d}\right) \text { and } \chi_{n} \stackrel{*}{\rightarrow} \chi \text { in } B V(A ;\{0,1\})\right\} .
\end{array}
$$

Let $V:\{0,1\} \times \mathbb{R}^{d \times N} \rightarrow(0,+\infty)$ be given by

$$
V(q, z):=q W_{1}(z)+(1-q) W_{2}(z)
$$

and $\overline{F_{\mathcal{O D}}}: B V(\Omega ;\{0,1\}) \times B V\left(\Omega ; \mathbb{R}^{d}\right) \times \mathcal{A}(\Omega) \rightarrow[0,+\infty]$ be defined as

$\overline{F_{\mathcal{O D}}}(\chi, u ; A):=\int_{A} Q V(\chi, \nabla u) d x+\int_{A} Q V^{\infty}\left(\chi, \frac{d D^{c} u}{d\left|D^{c} u\right|}\right) d\left|D^{c} u\right|+\int_{J_{(\chi, u)} \cap A} K_{2}\left(\chi^{+}, \chi^{-}, u^{+}, u^{-}, \nu\right) d \mathcal{H}^{N-1}$

where $Q V$ is the quasiconvex envelope of $V$ given in (3.2) $Q V^{\infty}$ is the recession function of $Q V$, namely,

$$
Q V^{\infty}(q, z):=\lim _{t \rightarrow \infty} \frac{Q V(q, t z)}{t}
$$

and

$$
K_{2}(a, b, c, d, \nu):=\inf \left\{\int_{Q_{\nu}} Q V^{\infty}(\chi(x), \nabla u(x)) d x+|D \chi|\left(Q_{\nu}\right):(\chi, u) \in \mathcal{A}_{2}(a, b, c, d, \nu)\right\}
$$

where

$$
\begin{aligned}
\mathcal{A}_{2}(a, b, c, d, \nu) & :=\left\{(\chi, u) \in B V\left(Q_{\nu} ;\{0,1\}\right) \times W^{1,1}\left(Q_{\nu} ; \mathbb{R}^{d}\right):\right. \\
& (\chi(y), u(y))=(a, c) \text { if } y \cdot \nu=\frac{1}{2},(\chi(y), u(y))=(b, d) \text { if } y \cdot \nu=-\frac{1}{2}, \\
& \left.(\chi, u) \text { are } 1-\text { periodic in } \nu_{1}, \ldots, \nu_{N-1} \text { directions }\right\},
\end{aligned}
$$

for $(a, b, c, d, \nu) \in\{0,1\} \times\{0,1\} \times \mathbb{R}^{d} \times \mathbb{R}^{d} \times S^{N-1}$, with $\left\{\nu_{1}, \nu_{2}, \ldots, \nu_{N-1}, \nu\right\}$ an orthonormal basis of $\mathbb{R}^{N}$ and $Q_{\nu}$ the unit cube, centered at the origin, with one direction parallel to $\nu$.

In Section 6 we obtain the following integral representation.

Theorem 1.1 Let $\Omega \subset \mathbb{R}^{N}$ be a bounded open set and let $W_{i}: \mathbb{R}^{d \times N} \rightarrow[0,+\infty), i=1,2$, be continuous functions satisfying (1.1). Let $\overline{F_{\mathcal{O D}}}$ be the functional defined in (1.5). Then for every $(\chi, u) \in L^{1}(\Omega ;\{0,1\}) \times$ $L^{1}\left(\Omega ; \mathbb{R}^{d}\right)$

$$
\mathcal{F}_{\mathcal{O D}}(\chi, u ; A)= \begin{cases}\overline{F_{\mathcal{O D}}}(\chi, u ; A) & \text { if }(\chi, u) \in B V(\Omega ;\{0,1\}) \times B V\left(\Omega ; \mathbb{R}^{d}\right), \\ +\infty & \text { otherwise. }\end{cases}
$$


This result will be achieved as a particular case of a more general theorem dealing with special functions of bounded variation which are piecewise constants.

In fact we provide an integral representation for the relaxation of the functional $F: L^{1}\left(\Omega ; \mathbb{R}^{m}\right) \times$ $L^{1}\left(\Omega ; \mathbb{R}^{d}\right) \times \mathcal{A}(\Omega) \rightarrow[0,+\infty]$ defined by

$$
F(v, u ; A):= \begin{cases}\int_{A} f(v, \nabla u) d x+\int_{A \cap J_{v}} g\left(v^{+}, v^{-}, \nu_{v}\right) d \mathcal{H}^{N-1} & \text { in } S B V_{0}\left(A ; \mathbb{R}^{m}\right) \times W^{1,1}\left(A ; \mathbb{R}^{d}\right), \\ +\infty & \text { otherwise, }\end{cases}
$$

where $S B V_{0}\left(A ; \mathbb{R}^{m}\right)$ is defined in (2.4) (see Section 2) and $f: \mathbb{R}^{m} \times \mathbb{R}^{d \times N} \rightarrow\left[0,+\infty\left[, g: \mathbb{R}^{m} \times \mathbb{R}^{m} \times S^{N-1} \rightarrow\right.\right.$ $[0,+\infty[$ satisfy the following hypotheses:

$\left(F_{1}\right) f$ is continuous;

$\left(F_{2}\right)$ there exist $0<\beta^{\prime} \leq \beta$ such that

$$
\beta^{\prime}|z| \leq f(q, z) \leq \beta(1+|z|)
$$

for every $(q, z) \in \mathbb{R}^{m} \times \mathbb{R}^{d \times N} ;$

$\left(F_{3}\right)$ there exists $L>0$ such that

$$
\left|f\left(q_{1}, z\right)-f\left(q_{2}, z\right)\right| \leq L\left|q_{1}-q_{2}\right|(1+|z|)
$$

for every $q_{1}, q_{2} \in \mathbb{R}^{m}$ and $z \in \mathbb{R}^{d \times N}$;

$\left(F_{4}\right)$ there exist $\alpha \in(0,1)$, and $C, L>0$ such that

$$
t|z|>L \Rightarrow\left|f^{\infty}(q, z)-\frac{f(q, t z)}{t}\right| \leq C \frac{|z|^{1-\alpha}}{t^{\alpha}}, \text { for every }(q, z) \in \mathbb{R}^{m} \times \mathbb{R}^{d \times N}, t \in \mathbb{R},
$$

with $f^{\infty}$ the recession function of $f$ with respect to the last variable, defined as

$$
f^{\infty}(q, z):=\limsup _{t \rightarrow \infty} \frac{f(q, t z)}{t},
$$

for every $(q, z) \in \mathbb{R}^{m} \times \mathbb{R}^{d \times N}$;

$\left(G_{1}\right) g$ is continuous;

$\left(G_{2}\right)$ there exists a constant $C>0$ such that

$$
\frac{1}{C}(1+|\lambda-\theta|) \leq g(\lambda, \theta, \nu) \leq C(1+|\lambda-\theta|),
$$

for every $(\lambda, \theta, \nu) \in \mathbb{R}^{m} \times \mathbb{R}^{m} \times S^{N-1}$,

$\left(G_{3}\right) g(\lambda, \theta, \nu)=g(\theta, \lambda,-\nu)$, for every $(\lambda, \theta, \nu) \in \mathbb{R}^{m} \times \mathbb{R}^{m} \times S^{N-1}$.

The relaxed localized energy of (1.9) is given by

$$
\begin{aligned}
& \mathcal{F}(v, u ; A):=\inf \left\{\liminf _{n \rightarrow \infty}\left(\int_{A} f\left(v_{n}, \nabla u_{n}\right) d x+\int_{J_{v_{n}} \cap A} g\left(v_{n}{ }^{+}, v_{n}{ }^{-}, \nu_{v_{n}}\right) d \mathcal{H}^{N-1}\right):\left\{u_{n}\right\} \subset W^{1,1}\left(A ; \mathbb{R}^{d}\right),\right. \\
& \left.\left\{v_{n}\right\} \subset S B V_{0}\left(A ; \mathbb{R}^{m}\right), u_{n} \rightarrow u \text { in } L^{1}\left(A ; \mathbb{R}^{d}\right) \text { and } v_{n} \rightarrow v \text { in } L^{1}\left(A ; \mathbb{R}^{m}\right)\right\} \text {. }
\end{aligned}
$$

Let $\overline{F_{0}}: S B V_{0}\left(\Omega ; \mathbb{R}^{m}\right) \times B V\left(\Omega ; \mathbb{R}^{d}\right) \times \mathcal{A}(\Omega) \rightarrow[0,+\infty]$ be given by

$$
\overline{F_{0}}(v, u ; A):=\int_{A} Q f(v, \nabla u) d x+\int_{A} Q f^{\infty}\left(v, \frac{d D^{c} u}{d\left|D^{c} u\right|}\right) d\left|D^{c} u\right|+\int_{J_{(v, u)} \cap A} K_{3}\left(v^{+}, v^{-}, u^{+}, u^{-}, \nu\right) d \mathcal{H}^{N-1},
$$


where $Q f$ is the quasiconvex envelope of $f$ given in (3.2), $Q f^{\infty}$ is the recession function of $Q f$, and $K_{3}$ : $\mathbb{R}^{m} \times \mathbb{R}^{m} \times \mathbb{R}^{d} \times \mathbb{R}^{d} \times S^{N-1} \rightarrow[0,+\infty[$ is defined as

$$
\begin{aligned}
& K_{3}(a, b, c, d, \nu):= \\
& \inf \left\{\int_{Q_{\nu}} Q f^{\infty}(v(x), \nabla u(x)) d x+\int_{J_{v} \cap Q_{\nu}} g\left(v^{+}(x), v^{-}(x), \nu(x)\right) d \mathcal{H}^{N-1}:(v, u) \in \mathcal{A}_{3}(a, b, c, d, \nu)\right\}
\end{aligned}
$$

where

$$
\begin{aligned}
\mathcal{A}_{3}(a, b, c, d, \nu):= & \left\{(v, u) \in\left(S B V_{0}\left(Q_{\nu} ; \mathbb{R}^{m}\right) \cap L^{\infty}\left(Q_{\nu} ; \mathbb{R}^{m}\right)\right) \times W^{1,1}\left(Q_{\nu} ; \mathbb{R}^{d}\right):\right. \\
& (v(y), u(y))=(a, c) \text { if } y \cdot \nu=\frac{1}{2},(v(y), u(y))=(b, d) \text { if } y \cdot \nu=-\frac{1}{2}, \\
& \left.(v, u) \text { are } 1-\text { periodic in } \nu_{1}, \ldots, \nu_{N-1} \text { directions }\right\},
\end{aligned}
$$

with $\left\{\nu_{1}, \nu_{2}, \ldots, \nu_{N-1}, \nu\right\}$ an orthonormal basis of $\mathbb{R}^{N}$.

In the following we present the main result.

Theorem 1.2 Let $\Omega \subset \mathbb{R}^{N}$ be a bounded open set and let $f: \mathbb{R}^{m} \times \mathbb{R}^{d \times N} \rightarrow[0,+\infty[$ be a function satisfying $\left(F_{1}\right)-\left(F_{4}\right)$ and $g: \mathbb{R}^{m} \times \mathbb{R}^{m} \times S^{N-1} \rightarrow\left[0,+\infty\left[\right.\right.$ satisfying $\left(G_{1}\right)-\left(G_{3}\right)$. Let $F$ be the functional defined in (1.9). Then for every $(v, u) \in L^{1}\left(\Omega ; \mathbb{R}^{m}\right) \times L^{1}\left(\Omega ; \mathbb{R}^{d}\right)$

$$
\mathcal{F}(v, u ; \Omega)= \begin{cases}\overline{F_{0}}(v, u ; \Omega) & \text { if }(v, u) \in S B V_{0}\left(\Omega ; \mathbb{R}^{m}\right) \times B V\left(\Omega ; \mathbb{R}^{d}\right), \\ +\infty & \text { otherwise. }\end{cases}
$$

The paper is organized as follows. Section 2 is devoted to preliminary results dealing with functions of bounded variation, perimeters and special functions of bounded variation which are piecewise constant. The properties of the energy densities and several auxiliary results involved in the proofs of representation Theorems 1.1 and 1.2 are discussed in Section 3 The proof of the lower bound for $\mathcal{F}$ in (1.11) is presented in Sections 4, while Section 5 contains the upper bound and the proof of Theorem 1.2. The applications to optimal design problems as in [6] and the comparison with previous related relaxation results as in [25], such as Theorem 1.1, are discussed in Section 6.

\section{Preliminaries}

We give a brief survey of functions of bounded variation and sets of finite perimeter.

In the following $\Omega \subset \mathbb{R}^{N}$ is an open bounded set and we denote by $\mathcal{A}(\Omega)$ the family of all open subsets of $\Omega$. The $N$-dimensional Lebesgue measure is designated as $\mathcal{L}^{N}$, while $\mathcal{H}^{N-1}$ denotes the $(N-1)$-dimensional Hausdorff measure. The unit cube in $\mathbb{R}^{N},\left(-\frac{1}{2}, \frac{1}{2}\right)^{N}$, is denoted by $Q$ and we set $Q\left(x_{0}, \varepsilon\right):=x_{0}+\varepsilon Q$ for $\varepsilon>0$. For every $\nu \in S^{N-1}$ we define $Q_{\nu}:=R_{\nu}(Q)$, where $R_{\nu}$ is a rotation such that $R_{\nu}\left(e_{N}\right)=\nu$. The constant $C$ may vary from line to line.

We denote by $\mathcal{M}(\Omega)$ the space of all signed Radon measures in $\Omega$ with bounded total variation. By the Riesz Representation Theorem, $\mathcal{M}(\Omega)$ can be identified to the dual of the separable space $\mathcal{C}_{0}(\Omega)$ of continuous functions on $\Omega$ vanishing on the boundary $\partial \Omega$. If $\lambda \in \mathcal{M}(\Omega)$ and $\mu \in \mathcal{M}(\Omega)$ is a nonnegative Radon measure, we denote by $\frac{d \lambda}{d \mu}$ the Radon-Nikodým derivative of $\lambda$ with respect to $\mu$.

The following version of Besicovitch Differentiation Theorem was proven by Ambrosio and Dal Maso 7 , Proposition 2.2].

Theorem 2.1 If $\lambda$ and $\mu$ are Radon measures in $\Omega, \mu \geq 0$, then there exists a Borel measure set $E \subset \Omega$ such that $\mu(E)=0$, and for every $x \in \operatorname{supp} \mu-E$

$$
\frac{d \lambda}{d \mu}(x):=\lim _{\varepsilon \rightarrow 0^{+}} \frac{\lambda(x+\varepsilon C)}{\mu(x+\varepsilon C)}
$$

exists and is finite whenever $C$ is a bounded, convex, open set containing the origin. 
We recall that the exceptional set $\mathrm{E}$ above does not depend on $\mathrm{C}$. An immediate corollary is the generalization of Lebesgue-Besicovitch Differentiation Theorem given below.

Theorem 2.2 If $\mu$ is a nonnegative Radon measure and if $f \in L_{\mathrm{loc}}^{1}\left(\mathbb{R}^{N}, \mu\right)$ then

$$
\lim _{\varepsilon \rightarrow 0^{+}} \frac{1}{\mu(x+\varepsilon C)} \int_{x+\varepsilon C}|f(y)-f(x)| d \mu(y)=0
$$

for $\mu$ - a.e. $x \in \mathbb{R}^{N}$ and for every, bounded, convex, open set $C$ containing the origin.

Definition 2.3 A function $w \in L^{1}\left(\Omega ; \mathbb{R}^{d}\right)$ is said to be of bounded variation, and we write $w \in B V\left(\Omega ; \mathbb{R}^{d}\right)$, if all its first distributional derivatives $D_{j} w_{i}$ belong to $\mathcal{M}(\Omega)$ for $1 \leq i \leq d$ and $1 \leq j \leq N$.

The matrix-valued measure whose entries are $D_{j} w_{i}$ is denoted by $D w$ and $|D w|$ stands for its total variation. We observe that if $w \in B V\left(\Omega ; \mathbb{R}^{d}\right)$ then $w \mapsto|D w|(\Omega)$ is lower semicontinuous in $B V\left(\Omega ; \mathbb{R}^{d}\right)$ with respect to the $L_{\text {loc }}^{1}\left(\Omega ; \mathbb{R}^{d}\right)$ topology.

We briefly recall some facts about functions of bounded variation. For more details we refer the reader to [8], 21], [27] and [32].

Definition 2.4 Let $w, w_{n} \in B V\left(\Omega ; \mathbb{R}^{d}\right)$. The sequence $\left\{w_{n}\right\}$ strictly converges in $B V\left(\Omega ; \mathbb{R}^{d}\right)$ to $w$ if $\left\{w_{n}\right\}$ converges to $w$ in $L^{1}\left(\Omega ; \mathbb{R}^{d}\right)$ and $\left\{\left|D w_{n}\right|(\Omega)\right\}$ converges to $|D w|(\Omega)$ as $n \rightarrow \infty$.

Definition 2.5 Given $w \in B V\left(\Omega ; \mathbb{R}^{d}\right)$ the approximate upper limit and the approximate lower limit of each component $w^{i}, i=1, \ldots, d$, are defined by

$$
\left(w^{i}\right)^{+}(x):=\inf \left\{t \in \mathbb{R}: \lim _{\varepsilon \rightarrow 0^{+}} \frac{\mathcal{L}^{N}\left(\left\{y \in \Omega \cap Q(x, \varepsilon): w^{i}(y)>t\right\}\right)}{\varepsilon^{N}}=0\right\}
$$

and

$$
\left(w^{i}\right)^{-}(x):=\sup \left\{t \in \mathbb{R}: \lim _{\varepsilon \rightarrow 0^{+}} \frac{\mathcal{L}^{N}\left(\left\{y \in \Omega \cap Q(x, \varepsilon): w^{i}(y)<t\right\}\right)}{\varepsilon^{N}}=0\right\},
$$

respectively. The jump set of $w$ is given by

$$
J_{w}:=\bigcup_{i=1}^{d}\left\{x \in \Omega:\left(w^{i}\right)^{-}(x)<\left(w^{i}\right)^{+}(x)\right\} .
$$

It can be shown that $J_{w}$ and the complement of the set of Lebesgue points of $w$ differ, at most, by a set of $\mathcal{H}^{N-1}$ measure zero. Moreover, $J_{w}$ is $(N-1)$-rectifiable, i.e., there are $C^{1}$ hypersurfaces $\Gamma_{i}$ such that $\mathcal{H}^{N-1}\left(J_{w} \backslash \cup_{i=1}^{\infty} \Gamma_{i}\right)=0$.

Proposition 2.6 If $w \in B V\left(\Omega ; \mathbb{R}^{d}\right)$ then

i) for $\mathcal{L}^{N}-$ a.e. $x \in \Omega$

$$
\lim _{\varepsilon \rightarrow 0^{+}} \frac{1}{\varepsilon}\left\{\frac{1}{\varepsilon^{N}} \int_{Q(x, \varepsilon)}|w(y)-w(x)-\nabla w(x) \cdot(y-x)|^{\frac{N}{N-1}} d y\right\}^{\frac{N-1}{N}}=0
$$

ii) for $\mathcal{H}^{N-1}$-a.e. $x \in J_{w}$ there exist $w^{+}(x), w^{-}(x) \in \mathbb{R}^{d}$ and $\nu(x) \in S^{N-1}$ normal to $J_{w}$ at $x$, such that

$$
\lim _{\varepsilon \rightarrow 0^{+}} \frac{1}{\varepsilon^{N}} \int_{Q_{\nu}^{+}(x, \varepsilon)}\left|w(y)-w^{+}(x)\right| d y=0, \quad \lim _{\varepsilon \rightarrow 0^{+}} \frac{1}{\varepsilon^{N}} \int_{Q_{\nu}^{-}(x, \varepsilon)}\left|w(y)-w^{-}(x)\right| d y=0,
$$

where $Q_{\nu}^{+}(x, \varepsilon):=\left\{y \in Q_{\nu}(x, \varepsilon):\langle y-x, \nu\rangle>0\right\}$ and $Q_{\nu}^{-}(x, \varepsilon):=\left\{y \in Q_{\nu}(x, \varepsilon):\langle y-x, \nu\rangle<0\right\}$; 
iii) for $\mathcal{H}^{N-1}$-a.e. $x \in \Omega \backslash J_{w}$

$$
\lim _{\varepsilon \rightarrow 0^{+}} \frac{1}{\varepsilon^{N}} \int_{Q(x, \varepsilon)}|w(y)-w(x)| d y=0
$$

We observe that in the vector-valued case in general $\left(w^{i}\right)^{ \pm} \neq\left(w^{ \pm}\right)^{i}$. In the sequel $w^{+}$and $w^{-}$denote the vectors introduced in $i i)$ above.

Choosing a normal $\nu_{w}(x)$ to $J_{w}$ at $x$, we denote the jump of $w$ across $J_{w}$ by $[w]:=w^{+}-w^{-}$. The distributional derivative of $w \in B V\left(\Omega ; \mathbb{R}^{d}\right)$ admits the decomposition

$$
D w=\nabla w \mathcal{L}^{N}\left\lfloor\Omega+\left([w] \otimes \nu_{w}\right) \mathcal{H}^{N-1}\left\lfloor J_{w}+D^{c} w\right.\right.
$$

where $\nabla w$ represents the density of the absolutely continuous part of the Radon measure $D w$ with respect to the Lebesgue measure. The Hausdorff, or jump, part of Dw is represented by $\left([w] \otimes \nu_{w}\right) \mathcal{H}^{N-1}\left\lfloor J_{w}\right.$ and $D^{c} w$ is the Cantor part of $D w$. The measure $D^{c} w$ is singular with respect to the Lebesgue measure and it is diffuse, i.e., every Borel set $B \subset \Omega$ with $\mathcal{H}^{N-1}(B)<\infty$ has Cantor measure zero.

The following result, that will be exploited in the sequel, can be found in [25, Lemma 2.6].

Lemma 2.7 Let $w \in B V\left(\Omega ; \mathbb{R}^{d}\right)$, for $\mathcal{H}^{N-1}$ a.e. $x$ in $J_{w}$,

$$
\lim _{\varepsilon \rightarrow 0^{+}} \frac{1}{\varepsilon^{N-1}} \int_{J_{w} \cap Q_{\nu(x)}(x, \varepsilon)}\left|w^{+}(y)-w^{-}(y)\right| d \mathcal{H}^{N-1}=\left|w^{+}(x)-w^{-}(x)\right| .
$$

In the following we give some preliminary notions related with sets of finite perimeter. For a detailed treatment we refer to [8].

Definition 2.8 Let $E$ be an $\mathcal{L}^{N}$ - measurable subset of $\mathbb{R}^{N}$. For any open set $\Omega \subset \mathbb{R}^{N}$ the perimeter of $E$ in $\Omega$, denoted by $P(E ; \Omega)$, is the variation of $\chi_{E}$ in $\Omega$, i.e.

$$
P(E ; \Omega):=\sup \left\{\int_{E} \operatorname{div} \varphi d x: \varphi \in C_{c}^{1}\left(\Omega ; \mathbb{R}^{d}\right),\|\varphi\|_{L^{\infty}} \leq 1\right\} .
$$

We say that $E$ is a set of finite perimeter in $\Omega$ if $P(E ; \Omega)<+\infty$.

Recalling that if $\mathcal{L}^{N}(E \cap \Omega)$ is finite, then $\chi_{E} \in L^{1}(\Omega)$, by [8, Proposition 3.6], it results that $E$ has finite perimeter in $\Omega$ if and only if $\chi_{E} \in B V(\Omega)$ and $P(E ; \Omega)$ coincides with $\left|D \chi_{E}\right|(\Omega)$, the total variation in $\Omega$ of the distributional derivative of $\chi_{E}$. Moreover, a generalized Gauss-Green formula holds:

$$
\int_{E} \operatorname{div} \varphi d x=\int_{\Omega}<\nu_{E}, \varphi>d\left|D \chi_{E}\right| \forall \varphi \in C_{c}^{1}\left(\Omega ; \mathbb{R}^{d}\right)
$$

where $D \chi_{E}=\nu_{E}\left|D \chi_{E}\right|$ is the polar decomposition of $D \chi_{E}$.

We also recall that, when dealing with sets of finite measure, a sequence of sets $\left\{E_{n}\right\}$ converges to $E$ in measure in $\Omega$ if $\mathcal{L}^{N}\left(\Omega \cap\left(E_{n} \Delta E\right)\right)$ converges to 0 as $n \rightarrow \infty$, where $\Delta$ stands for the symmetric difference. Analogously, the local convergence in measure corresponds to the above convergence in measure for any open set $A \subset \subset \Omega$. These convergences are equivalent to $L^{1}(\Omega)$ and $L_{\mathrm{loc}}^{1}(\Omega)$ convergences of the characteristic functions. We also remind that the local convergence in measure in $\Omega$ is equivalent to convergence in measure in domains $\Omega$ with finite measure.

Denoting by $\mathcal{P}(\Omega)$ the family of all sets with finite perimeters in $\Omega$ we recall the Fleming-Rishel formula (see [22, formula 4.59]): for every $\Phi \in W^{1,1}(\Omega)$ the set $\{t \in \mathbb{R}:\{\Phi>t\} \notin \mathcal{P}(\Omega)\}$ is negligible in $\mathbb{R}$ and

$$
\int_{\Omega} h|\nabla \Phi| d x=\int_{-\infty}^{+\infty} \int_{\partial^{*}\{\Phi>t\}} h d \mathcal{H}^{N-1} d t
$$

for every bounded Borel function $h: \Omega \rightarrow \mathbb{R}$, where $\partial^{*}\{\Phi>t\}$ denotes the essential boundary of $\{\Phi>t\}$ (cf. [8, Definition 3.60]).

At this point we deal with functions of bounded variation whose Cantor part is null. 
Definition 2.9 A function $v \in B V\left(\Omega ; \mathbb{R}^{m}\right)$ is said to be a special function of bounded variation, and we write $v \in S B V\left(\Omega ; \mathbb{R}^{m}\right)$, if $D^{c} v=\underline{0}$, i.e.

$$
D v=\nabla v \mathcal{L}^{N}\left\lfloor\Omega+\left([v] \otimes \nu_{v}\right) \mathcal{H}^{N-1}\left\lfloor J_{v} .\right.\right.
$$

The space $S B V_{0}\left(\Omega ; \mathbb{R}^{m}\right)$ is defined by

$$
S B V_{0}\left(\Omega ; \mathbb{R}^{m}\right):=\left\{v \in S B V\left(\Omega ; \mathbb{R}^{m}\right): \nabla v=0, \text { and } \mathcal{H}^{N-1}\left(J_{v}\right)<+\infty\right\} .
$$

Clearly, any characteristic function of a set of finite perimeter is in $S B V_{0}(\Omega)$.

We recall that a sequence of sets $\left\{E_{i}\right\}$ is a Borel partition of a Borel set $B \in \mathcal{B}\left(\mathbb{R}^{N}\right)$ if and only if

$$
E_{i} \in \mathcal{B}\left(\mathbb{R}^{N}\right) \text { for every } i, E_{i} \cap E_{j}=\emptyset \text { for every } i \neq j \text { and } \cup_{i=1}^{\infty} E_{i}=B \text {. }
$$

The above requirements could be weakened requiring that $\left|E_{i} \cap E_{j}\right|=0$, for $i \neq j$ and $\left|B \Delta \cup_{i=1}^{\infty} E_{i}\right|=0$. Such a sequence $\left\{E_{i}\right\}$ is said to be a Caccioppoli partition if and only if each $E_{i}$ is a set of finite perimeter.

The following result, whose proof can be found in [18, expresses the relations between Caccioppoli partitions and $S B V_{0}$ functions.

Lemma 2.10 If $v \in S B V_{0}\left(\Omega ; \mathbb{R}^{m}\right)$ then there exist a Borel partition $\left\{E_{i}\right\}$ of $\Omega$ and a sequence $\left\{v_{i}\right\} \subset \mathbb{R}^{m}$ such that

$$
\begin{gathered}
v=\sum_{i=1}^{\infty} v_{i} \chi_{E_{i}} \text { a e. } x \in \Omega, \\
\mathcal{H}^{N-1}\left(J_{v} \cap \Omega\right)=\frac{1}{2} \sum_{i=1}^{\infty} \mathcal{H}^{N-1}\left(\partial^{*} E_{i} \cap \Omega\right)=\frac{1}{2} \sum_{i \neq j=1}^{\infty} \mathcal{H}^{N-1}\left(\partial^{*} E_{i} \cap \partial^{*} E_{j} \cap \Omega\right), \\
\left(v^{+}, v^{-}, \nu_{v}\right) \equiv\left(v^{i}, v^{j}, \nu_{i}\right) \text { a.e. } x \in \partial^{*} E_{i} \cap \partial E_{j}^{*} \cap \Omega,
\end{gathered}
$$

$\nu_{i}$ being the unit normal to $\partial^{*} E_{i} \cap \partial E_{j}^{*}$,

In the sequel we identify $(v, u) \in S B V_{0}\left(\Omega ; \mathbb{R}^{m}\right) \times B V\left(\Omega ; \mathbb{R}^{d}\right)$ with their precise representatives $(\tilde{v}, \tilde{u})$. See [8, Definition 3.63 and Corollary 3.80] for the definition.

Remark 2.11 Since $S B V_{0}\left(\Omega ; \mathbb{R}^{m}\right) \subset B V\left(\Omega ; \mathbb{R}^{m}\right)$, then $(v, u) \in B V\left(\Omega ; \mathbb{R}^{m+d}\right)$ for every $(v, u) \in S B V_{0}\left(\Omega ; \mathbb{R}^{m}\right) \times$ $B V\left(\Omega ; \mathbb{R}^{d}\right)$. Thus $(v, u)$ is $\left|D^{c}(v, u)\right|$-measurable, and since $D^{c}(v, u)=\left(\underline{0}, D^{c} u\right)$, we may say that $v$ is $\left|D^{c} u\right|-$ measurable.

The following compactness result for bounded sequences in $S B V\left(\Omega ; \mathbb{R}^{m}\right)$ is due to Ambrosio (see [2], 4]).

Theorem 2.12 Let $\Phi:[0,+\infty) \rightarrow[0,+\infty), \Theta:(0,+\infty] \rightarrow(0,+\infty]$ be two functions, respectively convex and concave, and such that

$$
\begin{gathered}
\lim _{t \rightarrow \infty} \frac{\Phi(t)}{t}=+\infty, \quad \Phi \text { is nondecreasing, } \\
\Theta(+\infty)=\lim _{t \rightarrow \infty} \Theta(t), \quad \lim _{t \rightarrow 0^{+}} \frac{\Theta(t)}{t}=+\infty, \quad \Theta \text { is non decreasing. }
\end{gathered}
$$

Let $\left\{v_{n}\right\}$ be a sequence of functions in $S B V\left(\Omega ; \mathbb{R}^{m}\right)$ such that

$$
\sup _{n}\left\{\int_{\Omega} \Phi\left(\left|\nabla v_{n}\right|\right) d x+\int_{J_{v_{n}}} \Theta\left(\left|\left[v_{n}\right]\right|\right) d \mathcal{H}^{N-1}+\int_{\Omega}\left|v_{n}\right| d x\right\}<+\infty .
$$

Then there exists a subsequence $\left\{v_{n_{k}}\right\}$ converging in $L^{1}\left(\Omega ; \mathbb{R}^{m}\right)$ to a function $v \in S B V\left(\Omega ; \mathbb{R}^{m}\right)$, and

$$
\begin{gathered}
\nabla v_{n_{k}} \rightarrow \nabla v \quad \text { in } L^{1}\left(\Omega ; \mathbb{R}^{N \times m}\right), \quad\left[v_{n_{k}}\right] \otimes \nu_{v_{n_{k}}} \mathcal{H}^{N-1}\left\lfloorJ _ { v _ { n _ { k } } } \stackrel { * } { \rightarrow } [ v ] \otimes \nu _ { v } \mathcal { H } ^ { N - 1 } \left\lfloor J_{v},\right.\right. \\
\int_{J_{v} \cap \Omega} \Theta(|[v]|) d \mathcal{H}^{N-1} \leq \liminf _{n \rightarrow+\infty} \int_{J_{v_{n}} \cap \Omega} \Theta\left(\left|\left[v_{n}\right]\right|\right) d \mathcal{H}^{N-1} .
\end{gathered}
$$




\section{Auxiliary results}

This section is mainly devoted to describe the properties of the energy densities involved in the integral representation of relaxed functionals (1.5) and (1.12).

Recall that a Borel function $f: \mathbb{R}^{m} \times \mathbb{R}^{d \times N} \rightarrow[-\infty,+\infty]$ is said to be quasiconvex if

$$
f(q, z) \leq \frac{1}{\mathcal{L}^{N}(\Omega)} \int_{\Omega} f(q, z+\nabla \varphi(y)) d y
$$

for every open bounded set $\Omega \subset \mathbb{R}^{N}$ with $\mathcal{L}^{N}(\partial \Omega)=0$, for every $(q, z) \in \mathbb{R}^{m} \times \mathbb{R}^{d \times N}$ and every $\varphi \in$ $W_{0}^{1, \infty}\left(\Omega ; \mathbb{R}^{d}\right)$ whenever the right hand side of (3.1) exists as a Lebesgue integral.

The quasiconvex envelope of $f: \mathbb{R}^{m} \times \mathbb{R}^{d \times N} \rightarrow[0,+\infty]$ is the largest quasiconvex function below $f$ and it is denoted by $Q f$. If $f$ is Borel and locally bounded from below then it can be shown that

$$
Q f(q, z)=\inf \left\{\int_{Q} f(q, z+\nabla \varphi) d x: \varphi \in W_{0}^{1, \infty}\left(Q ; \mathbb{R}^{d}\right)\right\}
$$

for every $(q, z) \in \mathbb{R}^{m} \times \mathbb{R}^{d \times N}$.

The following result guarantees that the properties of $f$ are inherited by $Q f$. Since the proof develops along the lines as in [31, Proposition 2.2], in turn inspired by [19, we omit it.

Proposition 3.1 Let $f: \mathbb{R}^{m} \times \mathbb{R}^{d \times N} \rightarrow[0,+\infty)$ be a function satisfying $\left(F_{1}\right)-\left(F_{3}\right)$, and let $Q f: \mathbb{R}^{m} \times$ $\mathbb{R}^{d \times N} \rightarrow[0,+\infty)$ be its quasiconvexification, as in (3.2). Then $Q f$ satisfies $\left(F_{1}\right)-\left(F_{3}\right)$.

Remark 3.2 Let $f: \mathbb{R}^{m} \times \mathbb{R}^{d \times N} \rightarrow[0,+\infty)$ be a function satisfying $\left(F_{1}\right)-\left(F_{4}\right)$, with $f^{\infty}$ as in (1.10).

(i) Recall that the recession function $f^{\infty}(q, \cdot)$ is positively one homogeneous for every $q \in \mathbb{R}^{m}$.

(ii) We observe that, if $f$ satisfies the growth condition $\left(F_{2}\right)$, then $\beta^{\prime}|z| \leq f^{\infty}(q, z) \leq \beta|z|$ holds. Moreover, if $f$ satisfies $\left(F_{3}\right)$, then $f^{\infty}$ satisfies $\left|f^{\infty}(q, z)-f^{\infty}\left(q^{\prime}, z\right)\right| \leq L\left|q-q^{\prime}\right||z|$, where $L$ is the constant appearing in $\left(F_{3}\right)$.

(iii) As showed in [25, Remark 2.2 (ii)], if a function $f: \mathbb{R}^{m} \times \mathbb{R}^{d \times N} \longrightarrow[0,+\infty)$ is quasiconvex in the last variable and such that $f(q, z) \leq c(1+|z|)$, for some $c>0$, then, its recession function $f^{\infty}(q, \cdot)$ is also quasiconvex.

(iv) A proof entirely similar to [10, Proposition 3.4] (see also [31, Proposition 2.6]) ensures that for every $(q, z) \in \mathbb{R}^{m} \times \mathbb{R}^{d \times N}, Q\left(f^{\infty}\right)(q, z)=(Q f)^{\infty}(q, z)$, hence we will adopt the notation $Q f^{\infty}$. In particular if $f$ satisfies $\left(F_{1}\right)-\left(F_{3}\right)$, Proposition 3.1 guarantees that $Q f^{\infty}$ is continuous in both variables. Furthermore, for every $q \in \mathbb{R}^{m}, Q f^{\infty}(q, \cdot)$ is Lipschitz continuous in the last variable.

(v) $(Q f)^{\infty}$ satisfies the analogous condition to $\left(F_{4}\right)$. We also observe, as emphasized in [25], that $\left(F_{4}\right)$ is equivalent to say that there exist $C>0$ and $\alpha \in(0,1)$ such that

$$
\left|f^{\infty}(q, z)-f(q, z)\right| \leq C\left(1+|z|^{1-\alpha}\right)
$$

for every $(q, z) \in \mathbb{R}^{m} \times \mathbb{R}^{d \times N}$.

An argument entirely similar to [31, Proposition 2.7] ensures that there exist $\alpha \in(0,1)$, and $C^{\prime}>0$ such that

$$
\left|(Q f)^{\infty}(q, z)-Q f(q, z)\right| \leq C^{\prime}\left(1+|z|^{1-\alpha}\right)
$$

for every $(q, z) \in \mathbb{R}^{m} \times \mathbb{R}^{d \times N}$.

The following proposition, whose proof can be obtained arguing exactly as in [12, page 132], establishes the properties of the density $K_{3}$.

Proposition 3.3 Let $f: \mathbb{R}^{m} \times \mathbb{R}^{d \times N} \rightarrow[0,+\infty)$ and $g: \mathbb{R}^{m} \times \mathbb{R}^{m} \times S^{N-1} \rightarrow(0,+\infty)$. Let $K_{3}$ be the function defined in (1.13). If $\left(F_{1}\right)-\left(F_{4}\right)$ and $\left(G_{1}\right)-\left(G_{3}\right)$ hold then

a) $\left|K_{3}(a, b, c, d, \nu)-K_{3}\left(a^{\prime}, b^{\prime}, c^{\prime}, d^{\prime}, \nu\right)\right| \leq C\left(\left|a-a^{\prime}\right|+\left|b-b^{\prime}\right|+\left|c-c^{\prime}\right|+\left|d-d^{\prime}\right|\right)$ for every $(a, b, c, d, \nu)$, $\left(a^{\prime}, b^{\prime}, c^{\prime}, d^{\prime}, \nu\right) \in \mathbb{R}^{m} \times \mathbb{R}^{m} \times \mathbb{R}^{d} \times \mathbb{R}^{d} \times S^{N-1}$ 
b) $\nu \longmapsto K_{3}(a, b, c, d, \nu)$ is upper semicontinuous for every $(a, b, c, d) \in \mathbb{R}^{m} \times \mathbb{R}^{m} \times \mathbb{R}^{d} \times \mathbb{R}^{d}$;

c) $K_{3}$ is upper semicontinuous in $\mathbb{R}^{m} \times \mathbb{R}^{m} \times \mathbb{R}^{d} \times \mathbb{R}^{d} \times S^{N-1}$;

d) $K_{3}(a, b, c, d, \nu) \leq C(|a-b|+|c-d|+1)$ for every $\nu \in S^{N-1}$. More precisely, from the growth conditions $\left(F_{2}\right),\left(G_{2}\right)$ and the definition of $K_{3}$ we have $K_{3}(a, a, c, d, \nu) \leq C(|c-d|), K_{3}(a, b, c, c, \nu) \leq$ $C(1+|a-b|)$.

A Borel measurable function $g: \mathbb{R}^{m} \times \mathbb{R}^{m} \times S^{N-1} \rightarrow \mathbb{R}$ is BV-elliptic (cf. [3], 8] and [13]) if for all $(a, b, \nu) \in \mathbb{R}^{m} \times \mathbb{R}^{m} \times S^{N-1}$, and for any finite subset $T$ of $\mathbb{R}^{m}$

$$
\int_{J_{w} \cap Q_{\nu}} g\left(w^{+}, w^{-}, \nu_{w}\right) d \mathcal{H}^{N-1} \geq g(a, b, \nu)
$$

for all $w \in B V\left(Q_{\nu} ; T\right)$ such that $w=v_{0}$ on $\partial Q_{\nu}$, where

$$
v_{0}:=\left\{\begin{array}{l}
a \text { if } x \cdot \nu>0 \\
b \text { if } x \cdot \nu \leq 0
\end{array}\right.
$$

We are in position to provide some approximation results which allow us to reobtain the relaxed functionals and the related energy densities in terms of suitable relaxation procedures. To this end we start by stating a result very similar to [12, Proposition 3.5] which allows to achieve $K_{3}$.

Proposition 3.4 Let $f: \mathbb{R}^{m} \times \mathbb{R}^{d \times N} \rightarrow[0,+\infty)$ and $g: \mathbb{R}^{m} \times \mathbb{R}^{m} \times S^{N-1} \rightarrow(0,+\infty)$ be functions such that $\left(F_{1}\right)-\left(F_{4}\right)$ and $\left(G_{1}\right)-\left(G_{3}\right)$ hold, respectively. Let $K_{3}$ be the function defined in (1.13) and $\left(v_{0}, u_{0}\right)$ be given by

$$
v_{0}(x):=\left\{\begin{array}{l}
a \text { if } x \cdot \nu>0, \\
b \text { if } x \cdot \nu<0
\end{array}, \quad u_{0}(x):=\left\{\begin{array}{l}
c \text { if } x \cdot \nu>0 \\
d \text { if } x \cdot \nu<0
\end{array}\right.\right.
$$

Then

$$
\begin{aligned}
K_{3}(a, b, c, d, \nu) & =\inf _{\left(v_{n}, u_{n}\right)}\left\{\liminf _{n \rightarrow \infty}\left(\int_{Q_{\nu}} Q f^{\infty}\left(v_{n}(x), \nabla u_{n}(x)\right) d x+\int_{Q_{\nu} \cap J_{v_{n}}} g\left(v_{n}^{+}(x), v_{n}^{-}(x), \nu_{n}(x)\right) d \mathcal{H}^{N-1}\right):\right. \\
& \left.\left(v_{n}, u_{n}\right) \in S B V_{0}\left(Q_{\nu} ; \mathbb{R}^{m}\right) \times W^{1,1}\left(Q_{\nu} ; \mathbb{R}^{d}\right),\left(v_{n}, u_{n}\right) \rightarrow\left(v_{0}, u_{0}\right) \text { in } L^{1}\left(Q_{\nu} ; \mathbb{R}^{m+d}\right)\right\} \\
= & : K_{3}^{*}(a, b, c, d, \nu) .
\end{aligned}
$$

Remark 3.5 i) It is worthwhile to observe that the above result ensures a sharper result than the one which is stated, namely the same type of arguments in [12, Proposition 3.5] allow us to obtain $K_{3}(a, b, c, d, \nu)$ as a relaxation procedure but with test sequences in $\mathcal{A}_{3}(a, b, c, d, \nu)$, converging to $\left(v_{0}, u_{0}\right)$ in (3.5).

ii) Notice that by virtue of the growth conditions on $Q f^{\infty}$ (cf. Remark 3.2) we can replace in (1.14) the space $W^{1,1}\left(Q_{\nu} ; \mathbb{R}^{d}\right)$ by $W^{1, \infty}\left(Q_{\nu} ; \mathbb{R}^{d}\right)$.

iii) Under assumptions $\left(G_{1}\right)-\left(G_{3}\right)$, the function $K_{3}$ in (1.13) can be obtained, either taking test functions $v$ in $B V(\Omega ; T)$ for every $T \subset \mathbb{R}^{m}$, with card $(T)$ finite, or in $S B V_{0}\left(\Omega ; \mathbb{R}^{m}\right) \cap L^{\infty}\left(\Omega ; \mathbb{R}^{m}\right)$. This is easy to verify by virtue of Lemma 2.10. Namely, one can approximate functions $v$ in $S B V_{0}\left(\Omega ; \mathbb{R}^{m}\right) \cap L^{\infty}\left(\Omega ; \mathbb{R}^{m}\right)$ by sequences $\left\{v_{n}\right\}$ in $B V\left(\Omega ; T_{n}\right)$ with $T_{n} \subset \mathbb{R}^{m}$ and card $\left(T_{n}\right)$ finite. Moreover $\left(v_{n}^{+}, v_{n}^{-}, \nu_{v_{n}}\right) \rightarrow\left(v^{+}, v^{-}, \nu_{v}\right)$ pointwise and we can apply reverse Fatou's lemma to obtain the equivalence between the two possible definitions of $K_{3}$.

iv) Observe that the properties of $K_{3}$ and the assumptions on $f$ and $g$ allow us to replace in the definition of $\mathcal{A}_{3}$ (see formula (1.14) ) the set $S B V_{0}\left(Q ; \mathbb{R}^{m}\right) \cap L^{\infty}\left(\Omega ; \mathbb{R}^{m}\right)$ by $S B V_{0}\left(\Omega ; \mathbb{R}^{m}\right)$.

By the proposition below one can replace in (1.11), $f$ by its quasiconvexification $Q f$. We will omit the proof, which is quite standard, exploiting the relaxation results in the Sobolev spaces, cf. [19, Theorem 9.8]. 
Proposition 3.6 Let $\Omega \subset \mathbb{R}^{N}$ be a bounded open set, $f$ and $g$ be as in Theorem 4.1. Q $f$ as in (3.2) and let $\mathcal{F}$ be given by (1.11). Then for every $A \in \mathcal{A}(\Omega)$ and for every $(v, u) \in S B V_{0}\left(A ; \mathbb{R}^{m}\right) \times B V\left(A ; \mathbb{R}^{d}\right)$,

$$
\begin{gathered}
\mathcal{F}(v, u ; A)=\inf \left\{\liminf _{n \rightarrow \infty} \int_{A} Q f\left(v_{n}, \nabla u_{n}\right) d x+\int_{A \cap J_{v_{n}}} g\left(v_{n}^{+}, v_{n}^{-}, \nu_{n}\right) d \mathcal{H}^{N-1}:\right. \\
\left.\left\{\left(v_{n}, u_{n}\right)\right\} \subset S B V_{0}\left(A ; \mathbb{R}^{m}\right) \times W^{1,1}\left(A ; \mathbb{R}^{d}\right),\left(v_{n}, u_{n}\right) \rightarrow(v, u) \text { in } L^{1}\left(A ; \mathbb{R}^{m}\right) \times L^{1}\left(A ; \mathbb{R}^{d}\right)\right\} .
\end{gathered}
$$

The following result is analogous to [24, Proposition 2.4] and it is devoted to replace the test functions in (1.11) by smooth ones. We will omit the proof, and just observe that i) follows the arguments in [1] with the application of Morse's measure covering theorem (c.f. [23, Theorem 1.147]) .

Proposition 3.7 Let $f: \mathbb{R}^{m} \times \mathbb{R}^{d \times N} \rightarrow[0,+\infty]$ be a function satisfying $\left(F_{1}\right)-\left(F_{3}\right)$ and let $Q f$ be given by (3.2).

i) Let $B$ be a ball in $\mathbb{R}^{N}$. If

$$
\bar{F}_{0}(v, u ; B) \leq \liminf _{n \rightarrow \infty}\left(\int_{B} Q f\left(v_{n}, \nabla u_{n}\right) d x+\int_{J_{v_{n}} \cap B} g\left(v_{n}^{+}, v_{n}^{-}, \nu_{v_{n}}\right) d \mathcal{H}^{N-1}\right)
$$

holds for every $\left(v_{n}, u_{n}\right),(v, u) \in S B V_{0}\left(\Omega ; \mathbb{R}^{m}\right) \times W^{1,1}\left(\Omega ; \mathbb{R}^{d}\right)$ such that $\left(v_{n}, u_{n}\right) \rightarrow(v, u)$ in $L^{1}\left(\Omega ; \mathbb{R}^{m}\right) \times$ $L^{1}\left(\Omega ; \mathbb{R}^{d}\right)$ then it holds for all open bounded sets $\Omega \subset \mathbb{R}^{N}$.

ii) For every $(v, u) \in L^{1}\left(\Omega ; \mathbb{R}^{m}\right) \times L^{1}\left(\Omega ; \mathbb{R}^{d}\right),\left\{\left(v_{n}, u_{n}\right)\right\} \subset S B V_{0}\left(\Omega ; \mathbb{R}^{m}\right) \times W^{1,1}\left(\Omega ; \mathbb{R}^{d}\right)$ such that $\left(v_{n}, u_{n}\right) \rightarrow$ $(v, u)$ in $L^{1}\left(\Omega ; \mathbb{R}^{m}\right) \times L^{1}\left(\Omega ; \mathbb{R}^{d}\right)$ there exists $\left\{\left(\widetilde{v}_{n}, \widetilde{u}_{n}\right)\right\} \subset C_{0}^{\infty}\left(\mathbb{R}^{N} ; \mathbb{R}^{m}\right) \times C_{0}^{\infty}\left(\mathbb{R}^{N} ; \mathbb{R}^{d}\right)$ such that $\left(\widetilde{v}_{n}, \widetilde{u}_{n}\right) \rightarrow(v, u)$ strictly in $B V\left(\Omega ; \mathbb{R}^{m}\right) \times B V\left(\Omega ; \mathbb{R}^{d}\right)$ and

$$
\liminf _{n \rightarrow \infty} \int_{\Omega} Q f\left(\widetilde{v}_{n}, \nabla \widetilde{u}_{n}\right) d x=\liminf _{n \rightarrow \infty} \int_{\Omega} Q f\left(v_{n}, \nabla u_{n}\right) d x
$$

In order to achieve the integral representation in (1.2) for the jump part, we need to modify $\left\{\left(v_{n}, u_{n}\right)\right\}$ to match the boundary in such a way the new sequences will be in $\mathcal{A}_{3}\left(v^{+}(x), v^{-}(x), u^{+}\left(x_{0}\right), u^{-}\left(x_{0}\right), \nu\left(x_{0}\right)\right)$ given in (1.14), and the energy doesn't increase. This is achieved in the next Lemma that for sake of simplicity is stated in the unit cube $Q \subset \mathbb{R}^{N}$, and with the normal to the jump set $\nu=e_{N}$. The proof relies on the techniques of [15, Lemma 3.5], [25, Lemma 3.1] and [5, Lemma 4.4].

Lemma 3.8 Let $Q:=[0,1]^{N}$ and

$$
v_{0}(y):=\left\{\begin{array}{ll}
a & \text { if } x_{N}>0, \\
b & \text { if } x_{N}<0,
\end{array} \quad u_{0}(y):= \begin{cases}c & \text { if } x_{N}>0 \\
d & \text { if } x_{N}<0\end{cases}\right.
$$

Let $\left\{v_{n}\right\} \subset S B V_{0}\left(Q ; \mathbb{R}^{m}\right)$ and $\left\{u_{n}\right\} \subset W^{1,1}\left(Q ; \mathbb{R}^{d}\right)$, such that $v_{n} \rightarrow v_{0}$ in $L^{1}\left(Q ; \mathbb{R}^{m}\right)$ and $u_{n} \rightarrow u_{0}$ in $L^{1}\left(Q ; \mathbb{R}^{d}\right)$.

If $\rho$ is a mollifier, $\rho_{n}:=n^{N} \rho(n x)$, then there exists $\left\{\left(\zeta_{n}, \xi_{n}\right)\right\} \in \mathcal{A}_{3}\left(a, b, c, d, e_{N}\right)$ such that

$$
\begin{gathered}
\zeta_{n}=v_{0} \text { on } \partial Q, \zeta_{n} \rightarrow v_{0} \text { in } L^{1}\left(Q ; \mathbb{R}^{m}\right), \\
\xi_{n}=\rho_{i(n)} * u_{0} \text { on } \partial Q, \quad \xi_{n} \rightarrow u_{0} \text { in } L^{1}\left(Q ; \mathbb{R}^{d}\right)
\end{gathered}
$$

and

$$
\begin{aligned}
& \limsup _{n \rightarrow \infty}\left(\int_{Q} Q f\left(\zeta_{n}, \nabla \xi_{n}\right) d x+\int_{J_{\zeta_{n}} \cap Q} g\left(\zeta_{n}^{+}, \zeta_{n}^{-}, \nu_{\zeta_{n}}\right) d \mathcal{H}^{N-1}\right) \\
& \leq \liminf _{n \rightarrow \infty}\left(\int_{Q} Q f\left(v_{n}, \nabla u_{n}\right) d x+\int_{J_{v_{n}} \cap Q} g\left(v_{n}^{+}, v_{n}^{-}, \nu_{v_{n}}\right) d \mathcal{H}^{N-1}\right) .
\end{aligned}
$$


Proof. Without loss of generality, we may assume that

$$
\begin{aligned}
& \liminf _{n \rightarrow \infty}\left(\int_{Q} Q f\left(v_{n}, \nabla u_{n}\right) d x+\int_{J_{v_{n}} \cap Q} g\left(v_{n}^{+}, v_{n}^{-}, \nu_{v_{n}}\right) d \mathcal{H}^{N-1}\right) \\
& =\lim _{n \rightarrow \infty}\left(\int_{Q} Q f\left(v_{n}, \nabla u_{n}\right) d x+\int_{J_{v_{n}} \cap Q} g\left(v_{n}^{+}, v_{n}^{-}, \nu_{v_{n}}\right) d \mathcal{H}^{N-1}\right)<+\infty .
\end{aligned}
$$

The proof is divided in two steps.

Step 1. First we claim that for every $\varepsilon>0$, denoted $\left\|\left(v_{0}, u_{0}\right)\right\|_{\infty}$ by $M_{0}$, there exist a sequence $\left\{\bar{u}_{n}\right\} \subset$ $W^{1,1}\left(Q ; \mathbb{R}^{d}\right) \cap L^{\infty}\left(Q ; \mathbb{R}^{d}\right)$ and a sequence $\left\{\bar{v}_{n}\right\} \subset S B V_{0}\left(Q ; \mathbb{R}^{m}\right) \cap L^{\infty}\left(Q ; \mathbb{R}^{m}\right)$, and a constant $C>0$ such that $\left\|\bar{u}_{n}\right\|_{\infty},\left\|\bar{v}_{n}\right\|_{\infty} \leq C$ for every $n$ and

$$
\begin{aligned}
& \liminf _{n \rightarrow \infty}\left(\int_{Q} Q f\left(\bar{v}_{n}, \nabla \bar{u}_{n}\right) d x+\int_{J_{\bar{v}_{n}} \cap Q} g\left(\bar{v}_{n}{ }^{+}, \bar{v}_{n}{ }^{-}, \nu_{\bar{v}_{n}}\right) d \mathcal{H}^{N-1}\right) \\
& \leq \lim _{n \rightarrow \infty}\left(\int_{Q} Q f\left(v_{n}, \nabla u_{n}\right) d x+\int_{J_{v_{n}} \cap Q} g\left(v_{n}^{+}, v_{n}^{-}, \nu_{v_{n}}\right) d \mathcal{H}^{N-1}\right)+\varepsilon .
\end{aligned}
$$

To achieve the claim we can apply a truncation argument as in [15, Lemma 3.5], (c.f. also [12, Lemma 3.7]). For $a_{i} \in \mathbb{R}$ to be determined later depending on $\varepsilon$ and $M_{0}$, we define $\phi_{i} \in W_{0}^{1, \infty}\left(\mathbb{R}^{m+d} ; \mathbb{R}^{m+d}\right)$ such that

$$
\phi_{i}(x)= \begin{cases}x, & |x|<a_{i} \\ 0, & |x| \geq a_{i+1}\end{cases}
$$

$\left\|\nabla \phi_{i}\right\|_{\infty} \leq 1$, with $x \in \mathbb{R}^{m+d}$, and $x \equiv\left(x_{1}, x_{2}\right), x_{1} \in \mathbb{R}^{m}, x_{2} \in \mathbb{R}^{d}$.

For any $n \in \mathbb{N}$ and for any $i$ as above, let $\left(v_{n}^{i}, u_{n}^{i}\right) \in S B V_{0}\left(Q ; \mathbb{R}^{m}\right) \times W^{1,1}\left(Q ; \mathbb{R}^{d}\right) \cap L^{\infty}\left(Q ; \mathbb{R}^{m+d}\right)$ be given by

$$
\left(v_{n}^{i}, u_{n}^{i}\right):=\phi_{i}\left(v_{n}, u_{n}\right) .
$$

Considering the bulk part of the energy $F$ in (1.9), and exploiting Proposition 3.6 and the growth conditions on $f$ and $Q f$, we have

$$
\begin{aligned}
& \int_{Q} Q f\left(v_{n}^{i}, \nabla u_{n}^{i}\right) d x=\int_{Q \cap\left\{\left|\left(v_{n}, u_{n}\right)\right| \leq a_{i}\right\}} Q f\left(v_{n}, \nabla u_{n}\right) d x+\int_{Q \cap\left\{\left|\left(v_{n}, u_{n}\right)\right|>a_{i+1}\right\}} Q f(0,0) d x \\
& +\int_{Q \cap\left\{a_{i}<\left|\left(v_{n}, u_{n}\right)\right| \leq a_{i+1}\right\}} Q f\left(v_{n}^{i}, \nabla u_{n}^{i}\right) d x \\
& \leq \int_{Q} Q f\left(v_{n}, \nabla u_{n}\right) d x+C\left|Q \cap\left\{\left|\left(v_{n}, u_{n}\right)\right|>a_{i+1}\right\}\right|+C_{1} \int_{A \cap\left\{a_{i}<\left|\left(v_{n}, u_{n}\right)\right| \leq a_{i+1}\right\}}\left(1+\left|\nabla u_{n}\right|\right) d x .
\end{aligned}
$$

Concerning the surface term of the energy in (1.9), since $\left(\left(v_{n}^{i}\right)^{ \pm},\left(u_{n}^{i}\right)^{ \pm}\right)=\phi_{i}\left(v_{n}^{ \pm}, u_{n}^{ \pm}\right)$, and without loss of generality one can assume that $\left|\left(v_{n}^{-}, u_{n}^{-}\right)\right| \leq\left|\left(v_{n}^{+}, u_{n}^{+}\right)\right| \mathcal{H}^{N-1}$ - a.e. on $J_{\left(v_{n}, u_{n}\right)}$, we have that

$$
\begin{aligned}
& \int_{Q \cap J_{v_{n}^{i}}} g\left(\left(v_{n}^{i}\right)^{+},\left(v_{n}^{i}\right)^{-}, \nu_{v_{n}^{i}}\right) d \mathcal{H}^{N-1} \\
& \leq \int_{J_{v_{n}} \backslash\left\{a_{i+1} \leq\left|\left(v_{n}^{-}, u_{n}^{-}\right)\right|\right\} \cap Q} g\left(\phi_{i}\left(\left(v_{n}^{i}\right)^{+},\left(u_{n}^{i}\right)^{+}\right), \phi_{i}\left(\left(v_{n}^{i}\right)^{-},\left(u_{n}^{i}\right)^{-}\right), \nu_{\left(v_{n}^{i}, u_{n}^{i}\right)}\right) d \mathcal{H}^{N-1} .
\end{aligned}
$$

Arguing as in [15, Lemma 3.5] (cf. also [15, Remark 3.6]), and exploiting the growth conditions on $g$ we can 
estimate $\frac{1}{k} \sum_{i=1}^{k} F\left(v_{n}^{i}, u_{n}^{i} ; Q\right)$ for any fixed $k \in \mathbb{N}$, and for every $n \in \mathbb{N}$, with $k$ independent on $n$. Then

$$
\begin{aligned}
& \frac{1}{k} \sum_{i=1}^{k} F\left(v_{n}^{i}, u_{n}^{i} ; Q\right) \leq F\left(v_{n}, u_{n} ; Q\right)+\frac{1}{k} \sum_{i=2}^{k}\left(C\left|Q \cap\left\{\left|\left(v_{n}, u_{n}\right)\right|>a_{i+1}\right\}\right|+C_{4} \int_{J_{2}^{i} \cap Q}\left(1+\left|v_{n}^{-}\right|\right) d \mathcal{H}^{N-1}\right) \\
& +\frac{1}{k}\left(c_{2} \int_{Q}\left(1+\left|\nabla u_{n}\right|\right) d x+3 C_{4} \int_{J_{v_{n}} \cap Q}\left(1+\left|v_{n}^{+}-v_{n}^{-}\right|\right) d \mathcal{H}^{N-1}\right),
\end{aligned}
$$

where $J_{2}^{i}:=\left\{\left|v_{n}^{-}\right| \leq a_{i},\left|v_{n}^{+}\right| \geq a_{i+1}\right\}$. By the growth conditions there exists a constant $C$ such that

$$
\left(c_{2} \int_{Q}\left(1+\left|\nabla u_{n}\right|\right) d x+3 c_{4} \int_{J_{v_{n}} \cap Q}\left(1+\left|v_{n}^{+}-v_{n}^{-}\right|\right) d \mathcal{H}^{N-1}\right) \leq C,
$$

for every $n \in \mathbb{N}$. Choose $k \in \mathbb{N}$ such that $\frac{c}{k} \leq \frac{\varepsilon}{3}$. Moreover

$$
C \geq \int_{J_{2}^{i} \cap Q}\left|v_{n}^{+}-v_{n}^{-}\right| d \mathcal{H}^{N-1} \geq \int_{J_{2}^{i} \cap Q}\left(\left|v_{n}^{+}\right|-\left|v_{n}^{-}\right|\right) d \mathcal{H}^{N-1} \geq\left(a_{i+1}-a_{i}\right) \mathcal{H}^{N-1}\left(J_{2}^{i} \cap Q\right),
$$

whence

$$
\int_{J_{2}^{i} \cap Q}\left(1+\left|v_{n}^{-}\right|\right) d \mathcal{H}^{N-1} \leq C \frac{1+a_{i}}{a_{i+1}-a_{i}} .
$$

The sequence $\left\{a_{i}\right\}$ can be chosen recursively as follows

$$
\begin{aligned}
& C_{2}\left|Q \cap\left\{\left|\left(v_{n}, u_{n}\right)\right|>a_{i}\right\}\right| \leq \frac{\varepsilon}{3}, \text { for every } n \in \mathbb{N}, a_{i+1} \geq M_{0}, \\
& c_{4} C \frac{1+a_{i}}{a_{i+1}-a_{i}} \leq \frac{\varepsilon}{3} \text { for every } i \in \mathbb{N},
\end{aligned}
$$

which is possible since $\left\{\left(v_{n}, u_{n}\right)\right\}$ is bounded in $L^{1}$. Thus we obtain

$$
\frac{1}{k} \sum_{j=1}^{k} F\left(v_{n}^{i_{j}}, u_{n}^{i_{j}} ; Q\right) \leq F\left(v_{n}, u_{n} ; Q\right)+\varepsilon .
$$

Therefore for every $n \in \mathbb{N}$ there exists $i(n) \in\{1, \ldots, k\}$ such that

$$
F\left(v_{n}^{i_{n}}, u_{n}^{i_{n}} ; Q\right) \leq F\left(v_{n}, u_{n} ; Q\right)+\varepsilon .
$$

It suffices to define $\bar{v}_{n}:=v_{n}^{i_{n}}$ and $\bar{u}_{n}:=u_{n}^{i_{n}}$ to achieve (3.7) and observe that $\left\{\bar{u}_{n}\right\}$ and $\left\{\bar{v}_{n}\right\}$ are bounded in $L^{\infty}$, by construction.

Step 2. This step is devoted to the construction of sequences $\left\{\xi_{n}\right\}$ and $\left\{\zeta_{n}\right\}$ as in the statement. Let $\bar{v}_{n}$ and $\bar{u}_{n}$ be as in $i$ ). Define

$$
w_{n}(x):=\left(\rho_{n} * u_{0}\right)(x)=\int_{B\left(x, \frac{1}{n}\right)} \rho_{n}(x-y) u_{0}(y) d y .
$$

As $\rho$ is a mollifier, we have for each tangential direction $i=1, \ldots, N-1, w_{n}\left(x+e_{i}\right)=w_{n}(x)$ and so

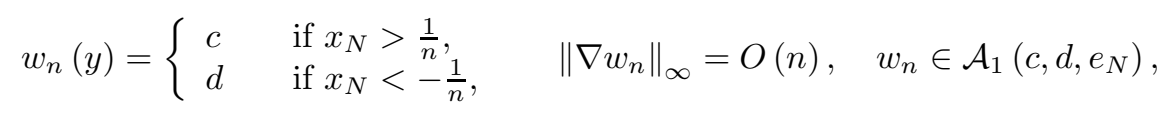

where

$$
\mathcal{A}_{1}\left(c, d, e_{N}\right):=\left\{u \in W^{1,1}\left(Q_{\nu} ; \mathbb{R}^{d}\right): u(y)=c \text { if } y \cdot \nu=\frac{1}{2}, u(y)=d \text { if } y \cdot \nu=-\frac{1}{2},\right.
$$

with $u 1-$ periodic in $\nu_{1}, \ldots, \nu_{N-1}$ directions $\}$. 
Let $\alpha_{n}:=\sqrt{\left\|\bar{u}_{n}-w_{n}\right\|_{L^{1}\left(Q ; \mathbb{R}^{d}\right)}+\left\|\bar{v}_{n}-v_{0}\right\|_{L^{1}(Q)}}$,

$k_{n}:=n\left[1+\left\|\bar{u}_{n}\right\|_{W^{1,1}\left(Q ; \mathbb{R}^{d}\right)}+\left\|w_{n}\right\|_{W^{1,1}\left(Q ; \mathbb{R}^{d}\right)}+\left\|\bar{v}_{n}\right\|_{B V(Q)}+\left\|v_{0}\right\|_{B V(Q)}+\mathcal{H}^{N-1}\left(J_{\bar{v}_{n}}\right)\right], s_{n}:=\frac{\alpha_{n}}{k_{n}}$ where $[k]$ denotes the largest integer less than or equal to $k$. Since $\alpha_{n} \rightarrow 0^{+}$, we may assume that $0 \leq \alpha_{n}<1$, and set $Q_{0}:=\left(1-\alpha_{n}\right) Q, Q_{i}:=\left(1-\alpha_{n}+i s_{n}\right) Q, i=1, \ldots, k_{n}$.

Consider a family of cut-off functions $\varphi_{i} \in C_{0}^{\infty}\left(Q_{i}\right), 0 \leq \varphi_{i} \leq 1, \varphi_{i}=1$ in $Q_{i-1},\left\|\nabla \varphi_{i}\right\|_{\infty}=O\left(\frac{1}{s_{n}}\right)$ for $i=1, \ldots, k_{n}$, and define

$$
u_{n}^{(i)}(x):=\left(1-\varphi_{i}(x)\right) w_{n}(x)+\varphi_{i}(x) \bar{u}_{n}(x) .
$$

Since $u_{n}^{(i)}=w_{n}$ on $\partial Q$ we have that $u_{n}^{(i)} \in \mathcal{A}_{1}\left(c, d, e_{N}\right)$. Clearly,

$$
\nabla u_{n}^{(i)}=\nabla \bar{u}_{n} \text { in } Q_{i-1}, \quad \nabla u_{n}^{(i)}=\nabla w_{n} \text { in } Q \backslash Q_{i},
$$

and in $Q_{i} \backslash Q_{i-1}$

$$
\nabla u_{n}^{(i)}=\nabla w_{n}+\varphi_{i}\left(\nabla \bar{u}_{n}-\nabla w_{n}\right)+\left(\bar{u}_{n}-w_{n}\right) \otimes \nabla \varphi_{i}
$$

For $0<t<1$ define

$$
v_{n, i}^{t}(x):= \begin{cases}v_{0}(x) & \text { if } \varphi_{i}(x)<t \\ \bar{v}_{n}(x) & \text { if } \varphi_{i}(x) \geq t\end{cases}
$$

Clearly, $\lim _{n \rightarrow \infty}\left\|v_{n, i}^{t}-v_{0}\right\|_{L^{1}(Q)}=0$ as $n \rightarrow \infty$, independently on $i$ and $t$. For every $n$ and $i$, by FlemingRishel formula (2.3) it is possible to find $\left.t_{n, i} \in\right] 0,1[$ such that

$$
\begin{aligned}
\left\{x \in Q: \varphi_{i}(x)<t_{n, i}\right\} & \in \mathcal{P}(Q), \\
\mathcal{H}^{N-1}\left(J_{v_{0}} \cap\left\{x \in Q: \varphi_{i}(x)=t_{n, i}\right\}\right) & =\mathcal{H}^{N-1}\left(J_{\bar{v}_{n}} \cap\left\{x \in Q: \varphi_{i}(x)=t_{n, i}\right\}\right)=0,
\end{aligned}
$$

where $\mathcal{P}(Q)$ denotes the family of sets with finite perimeter in $Q$. Let

$$
v_{n, i}^{t_{n, i}}:= \begin{cases}v_{0}(x) & \text { in } Q \cap\left\{x \in Q: \varphi_{i}(x)<t_{n, i}\right\} \\ \bar{v}_{n}(x) & \text { in } Q \cap\left\{x \in Q: \varphi_{i}(x) \geq t_{n, i}\right\}\end{cases}
$$

Clearly, $\lim _{n \rightarrow \infty}\left\|v_{n, i}^{t_{n, i}}-v_{0}\right\|_{L^{1}(Q)}=0,\left\{v_{n, i}^{t_{n, i}}\right\} \subset S B V_{0}\left(Q ; \mathbb{R}^{m}\right) \cap L^{\infty}\left(Q ; \mathbb{R}^{m}\right)$ and, from Step 1, it is uniformly bounded on $n, i$ and $t$.

We have

$$
\begin{aligned}
& \int_{Q} Q f\left(v_{n, i}^{t_{n, i}}, \nabla u_{n}^{(i)}\right) d x+\int_{J_{v_{n, i}} \cap Q} g\left(\left(v_{n, i}^{t_{n, i}}\right)^{+},\left(v_{n, i}^{t_{n, i}}\right)^{-}, \nu_{v_{n, i}^{t_{n, i}}}\right) d \mathcal{H}^{N-1} \\
& \leq \int_{Q} Q f\left(\bar{v}_{n}, \nabla \bar{u}_{n}\right) d x+C \int_{Q_{i} \backslash Q_{i-1}}\left(1+\left|\bar{u}_{n}(x)-w_{n}(x)\right| \frac{1}{s_{n}}+\left|\nabla \bar{u}_{n}(x)\right|+\left|\nabla w_{n}(x)\right|\right) d x \\
& +C \int_{Q \backslash Q_{i}}\left(1+\left|\nabla w_{n}(x)\right|\right) d x+\int_{Q \cap\left\{\varphi_{i}>t_{n, i}\right\}_{1}} g\left(\bar{v}_{n}{ }^{+}, \bar{v}_{n}{ }^{-}, \nu_{\bar{v}_{n}}\right) d \mathcal{H}^{N-1} \\
& +\left|D v_{n, i}^{t_{n, i}}\right|\left(\left(Q \cap\left\{\varphi_{i}>t_{n, i}\right\}_{0}\right)\right)+\mathcal{H}^{N-1}\left(\left(Q \cap\left\{\varphi_{i}>t_{n, i}\right\}_{0}\right)\right)+\left|D v_{n, i}^{t_{n, i}}\right|\left(\partial^{*}\left\{\varphi_{i}<t_{n, i}\right\}\right) \\
& +\mathcal{H}^{N-1}\left(\partial^{*}\left\{\varphi_{i}<t_{n, i}\right\}\right) \\
& \leq \int_{Q} Q f\left(\bar{v}_{n}, \nabla \bar{u}_{n}\right) d x+I_{1}+\int_{Q \cap J_{\bar{v}_{n}}} g\left(\bar{v}_{n}{ }^{+}, \bar{v}_{n}{ }^{-}, \nu \bar{v}_{n}\right) d \mathcal{H}^{N-1}+C\left|D v_{0}\right|\left(Q \backslash Q_{i}:\left\{\varphi_{i}>t_{n, i}\right\}_{0}\right) \\
& +\frac{C}{s_{n}} \int_{Q_{i} \backslash Q_{i-1}}\left|\bar{v}_{n}-v_{0}\right| d x+\frac{1}{s_{n}} O\left(s_{n}\right),
\end{aligned}
$$

where

$$
\left\{\varphi_{i}>t_{n, i}\right\}_{1}:=\left\{x \in Q: \frac{\left|\left\{x \in Q: \varphi_{i}>t_{n, i}\right\} \cap B_{\rho}(x)\right|}{\left|B_{\rho}(x)\right|}=1\right\}
$$


$\left\{\varphi_{i}>t_{n, i}\right\}_{0}:=\left\{x \in Q: \frac{\left|\left\{x \in Q: \varphi_{i}>t_{n, i}\right\} \cap B_{\rho}(x)\right|}{\left|B_{\rho}(x)\right|}=0\right\}$,
$I_{1}:=C \int_{Q_{i} \backslash Q_{i-1}}\left(1+\left|\bar{u}_{n}(x)-w_{n}(x)\right| \frac{1}{s_{n}}+\left|\nabla \bar{u}_{n}(x)\right|+\left|\nabla w_{n}(x)\right|\right) d x+C \int_{Q \backslash Q_{i}}\left(1+\left|\nabla w_{n}(x)\right|\right) d x$, and we have used (2.3) in the last two terms of the above estimate.

Averaging over all layers $Q_{i} \backslash Q_{i-1}$ one obtains

$$
\begin{aligned}
& \frac{1}{k_{n}} \sum_{i=1}^{k_{n}}\left(\int_{Q} Q f\left(v_{n, i}^{t_{n, i}}, \nabla u_{n}^{(i)}\right) d x+\int_{Q \cap J_{v_{n, i}} t_{n, i}} g\left(\left(v_{n, i}^{t_{n, i}}\right)^{+},\left(v_{n, i}^{t_{n_{n}}}\right)^{-}, \nu_{v_{n_{i}}}^{t_{n, i}}\right) d \mathcal{H}^{N-1}\right) \\
& \leq \int_{Q} Q f\left(\bar{v}_{n}, \bar{u}_{n}\right) d x+\int_{Q \cap J_{v_{n}}} g\left(\bar{v}_{n}^{+}, \bar{v}_{n}^{-}, \nu_{\bar{v}_{n}}\right) d \mathcal{H}^{N-1}+\frac{C}{k_{n}} \int_{Q}\left(1+\left|\nabla \bar{u}_{n}\right|+\left|\nabla \bar{v}_{n}\right|\right) d x \\
& +\frac{C}{k_{n}} \int_{Q}\left|\bar{u}_{n}-w_{n}\right| \frac{1}{s_{n}} d x+C \int_{Q \backslash Q_{0}}\left(1+\left|\nabla w_{n}\right|\right) d x+C\left|D v_{0}\right|\left(Q \backslash Q_{0}\right)+\frac{C}{s_{n} k_{n}} \int_{Q \backslash Q_{0}}\left|\bar{v}_{n}-v_{0}\right| d x+\frac{C}{k_{n}} \\
& \leq \int_{Q} Q f\left(\bar{v}_{n}, \nabla \bar{u}_{n}\right) d x+\int_{Q \cap J_{v_{n}}} g\left(\bar{v}_{n}{ }^{+}, \bar{v}_{n}{ }^{-}, \nu_{\bar{v}_{n}}\right) d \mathcal{H}^{N-1}+\frac{C}{k_{n}} \int_{Q}\left(1+\left|\nabla \bar{u}_{n}\right|+\left|\nabla \bar{v}_{n}\right|\right) d x \\
& +\frac{C}{\alpha_{n}}\left\|\bar{u}_{n}-w_{n}\right\|_{L^{1}}+C \int_{Q \backslash Q_{0}}\left(1+\left|\nabla w_{n}\right|\right) d x+C\left|D v_{0}\right|\left(Q \backslash Q_{0}\right)+\frac{C}{\alpha_{n}}\left\|\bar{v}_{n}-v_{0}\right\|_{L^{1}(Q)}+\frac{C}{k_{n} .}
\end{aligned}
$$

Since $\left|Q \backslash Q_{0}\right|=O\left(\alpha_{n}\right)$ and $\nabla w_{n}(x)=0$ if $\left|x_{N}\right|>\frac{1}{N}$ we estimate

$$
\int_{Q \backslash Q_{0}}\left(1+\left|\nabla w_{n}\right|\right) d x \leq O\left(\alpha_{n}\right)+\mathcal{H}^{N-1}\left(Q \backslash Q_{0} \cap\left\{x_{N}=0\right\}\right) \int_{-\frac{1}{n}}^{\frac{1}{n}} O(n) d x_{N}=O\left(\alpha_{n}\right) .
$$

The same argument exploited above in order to estimate $\int_{Q \backslash Q_{0}} d x$ applies to estimate $\left|D v_{0}\right|\left(Q \backslash Q_{0}\right)$ since $v_{0}$ is a jump function across $x_{N}=0$, namely $\left|D v_{0}\right|\left(Q \backslash Q_{0}\right)=C \mathcal{H}^{N-1}\left(Q \backslash Q_{0} \cap\left\{x_{N}=0\right\}\right)$, recalling also that $Q_{0}=\alpha_{n} Q$.

Setting $\varepsilon_{n}:=O\left(\frac{1}{n}\right)+C \sqrt{\left\|\bar{u}_{n}-w_{n}\right\|_{L^{1}\left(Q ; \mathbb{R}^{d}\right)}+\left\|\bar{v}_{n}-v_{0}\right\|_{L^{1}(Q)}}+O\left(\alpha_{n}\right)$ we have that $\varepsilon_{n} \rightarrow 0^{+}$and

$$
\begin{aligned}
& \frac{1}{k_{n}} \sum_{i=1}^{k_{n}}\left(\int_{Q} Q f\left(v_{n, i}^{t_{n, i}}, \nabla u_{n}^{(i)}\right) d x+\int_{Q \cap J_{v_{n, i}}} g\left(\left(v_{n, i}^{t_{n, i}}\right)^{+},\left(v_{n, i}^{t_{n, i}}\right)^{-}, \nu_{v_{n, i}}^{t_{n, i}}\right) d \mathcal{H}^{N-1}\right) \\
& \leq \int_{Q} Q f\left(\bar{v}_{n}, \nabla \bar{u}_{n}\right) d x+\int_{Q \cap J_{\bar{v}_{n}}} g\left(\bar{v}_{n}{ }^{+}, \bar{v}_{n}{ }^{-}, \nu_{\bar{v}_{n}}\right) d \mathcal{H}^{N-1}+\varepsilon_{n}
\end{aligned}
$$

and so there exists an index $i(n) \in\left\{1, \ldots, k_{n}\right\}$ for which

$$
\begin{aligned}
& \int_{Q} Q f\left(v_{n, i(n)}^{t_{n, i(n)}}, \nabla u_{n}^{i(n)}\right) d x+\int_{\substack{Q \cap J_{t_{n, i}} \\
v_{n_{i}}}} g\left(\left(v_{n, i}^{t_{n, i}}\right)^{+},\left(v_{n, i}^{t_{n, i}}\right)^{-}, \nu_{v_{n, i}}^{t_{n, i}}\right) d \mathcal{H}^{N-1} \\
& \leq \int_{Q} Q f\left(\bar{v}_{n}, \nabla \bar{u}_{n}\right) d x+\int_{Q \cap J_{\bar{v}_{n}}} g\left(\bar{v}_{n}{ }^{+} \cdot \bar{v}_{n}{ }^{-}, \nu_{\bar{v}_{n}}\right) d \mathcal{H}^{N-1}+\varepsilon_{n} .
\end{aligned}
$$

It suffices to define $\xi_{n}:=u_{n}^{i(n)}, \zeta_{n}:=v_{n, i(n)}^{t_{n, i(n)}}$ to get

$$
\begin{aligned}
& \limsup _{n \rightarrow \infty}\left(\int_{Q} Q f\left(\zeta_{n}, \nabla \xi_{n}\right) d x+\int_{J_{\zeta_{n}} \cap Q} g\left(\zeta_{n}^{+}, \zeta_{n}^{-}, \nu_{\zeta_{n}}\right) d \mathcal{H}^{N-1}\right) \\
& \leq \liminf _{n \rightarrow \infty}\left(\int_{Q} Q f\left(\bar{v}_{n}, \nabla \bar{u}_{n}\right) d x+\int_{J_{\bar{v}_{n}} \cap Q} g\left(\bar{v}_{n}^{+}, \bar{v}_{n}{ }^{-}, \nu_{\bar{v}_{n}}\right) d \mathcal{H}^{N-1}\right),
\end{aligned}
$$

which concludes the proof. 
Remark 3.9 i) Observe that arguing as in the first step of Lemma 3.8, it results that for every $u \in$ $B V\left(\Omega ; \mathbb{R}^{d}\right)$ and $v \in S B V_{0}\left(\Omega ; \mathbb{R}^{m}\right) \cap L^{\infty}\left(\Omega ; \mathbb{R}^{m}\right)$

$$
\begin{aligned}
\mathcal{F}(v, u ; A)= & \inf \left\{\liminf _{n \rightarrow \infty}\left(\int_{A} f\left(v_{n}, \nabla u_{n}\right) d x+\int_{J_{v_{n}} \cap A} g\left(v_{n}{ }^{+}, v_{n}{ }^{-}, \nu_{v_{n}}\right) d \mathcal{H}^{N-1}\right):\right. \\
& \left\{v_{n}\right\} \subset S B V_{0}\left(A ; \mathbb{R}^{m}\right) \cap L^{\infty}\left(A ; \mathbb{R}^{m}\right),\left\{u_{n}\right\} \subset W^{1,1}\left(A ; \mathbb{R}^{d}\right), \\
& \left.\left(v_{n}, u_{n}\right) \rightarrow(v, u) \text { in } L^{1}\left(A ; \mathbb{R}^{m+d}\right), \sup _{n}\left\|v_{n}\right\|_{\infty}<+\infty\right\} .
\end{aligned}
$$

ii) Similarly, if also $u \in B V\left(\Omega ; \mathbb{R}^{d}\right) \cap L^{\infty}\left(\Omega ; \mathbb{R}^{d}\right)$, then

$$
\begin{aligned}
\mathcal{F}(v, u ; A)= & \inf \left\{\liminf _{n \rightarrow \infty}\left(\int_{A} f\left(v_{n}, \nabla u_{n}\right) d x+\int_{J_{v_{n}} \cap A} g\left(v_{n}{ }^{+}, v_{n}{ }^{-}, \nu_{v_{n}}\right) d \mathcal{H}^{N-1}\right):\right. \\
& \left\{v_{n}\right\} \subset S B V_{0}\left(A ; \mathbb{R}^{m}\right) \cap L^{\infty}\left(A ; \mathbb{R}^{m}\right),\left\{u_{n}\right\} \subset W^{1,1}\left(A ; \mathbb{R}^{d}\right) \cap L^{\infty}\left(A ; \mathbb{R}^{d}\right), \\
& \left.\left(v_{n}, u_{n}\right) \rightarrow(v, u) \text { in } L^{1}\left(A ; \mathbb{R}^{m+d}\right), \sup _{n}\left\|\left(v_{n}, u_{n}\right)\right\|_{\infty}<+\infty\right\} .
\end{aligned}
$$

iii) Notice that an argument entirely similar to [13, Lemmas 13 and 14] allows us to say that for every $(v, u) \in S B V_{0}\left(\Omega ; \mathbb{R}^{m}\right) \times B V\left(\Omega ; \mathbb{R}^{d}\right)$, it results

$$
\mathcal{F}(v, u ; A)=\lim _{j \rightarrow \infty} \mathcal{F}\left(\phi_{j}(v, u) ; A\right),
$$

where $\phi_{j}$ are the functions defined in (3.8).

We conclude this section with a result that will be exploited in the sequel.

Lemma 3.10 Let $X$ be a function space, for any $F: \mathbb{R} \times X \rightarrow[0, \infty]$

$$
\limsup _{\varepsilon \rightarrow 0^{+}} \inf _{u \in X} F(\varepsilon, u) \leq \inf _{u \in X} \limsup _{\varepsilon \rightarrow 0^{+}} F(\varepsilon, u) .
$$

Proof. For any $\widetilde{u} \in X$

$$
\inf _{u \in X} F(\varepsilon, u) \leq F(\varepsilon, \widetilde{u}) .
$$

Thus

$$
\limsup _{\varepsilon \rightarrow 0^{+}} \inf _{u \in X} F(\varepsilon, u) \leq \limsup _{\varepsilon \rightarrow 0^{+}} F(\varepsilon, \widetilde{u})
$$

for every $\widetilde{u} \in X$. Applying the infimum in the previous inequality one obtains

$$
\inf _{\widetilde{u} \in X} \limsup _{\varepsilon \rightarrow 0^{+}} \inf _{u \in X} F(\varepsilon, u) \leq \inf _{\widetilde{u} \in X} \limsup _{\varepsilon \rightarrow 0^{+}} F(\varepsilon, \widetilde{u}) .
$$

Hence

$$
\limsup _{\varepsilon \rightarrow 0^{+}} \inf _{u \in X} F(\varepsilon, u) \leq \inf _{u \in X} \limsup _{\varepsilon \rightarrow 0^{+}} F(\varepsilon, u) .
$$

\section{Lower bound}

This section is devoted to the proof of the lower bound inequality for Theorem 1.2 Recall that $\mathcal{F}$ and $\overline{F_{0}}$ are the functionals introduced in (1.11) and (1.12). 
Theorem 4.1 Let $\Omega \subset \mathbb{R}^{N}$ be a bounded open set, let $f: \mathbb{R}^{m} \times \mathbb{R}^{d} \rightarrow[0,+\infty)$ satisfy $\left(F_{1}\right)-\left(F_{4}\right)$ and let $g: \mathbb{R}^{m} \times \mathbb{R}^{m} \times S^{N-1} \rightarrow[0,+\infty)$ satisfy $\left(G_{1}\right)-\left(G_{3}\right)$. Then for every $(v, u) \in S B V_{0}\left(\Omega ; \mathbb{R}^{m}\right) \times B V\left(\Omega ; \mathbb{R}^{d}\right)$, and for every sequence $\left\{\left(v_{n}, u_{n}\right)\right\} \subset S B V_{0}\left(\Omega ; \mathbb{R}^{m}\right) \times W^{1,1}\left(\Omega ; \mathbb{R}^{d}\right)$ such that $\left(v_{n}, u_{n}\right) \rightarrow(v, u)$ in $L^{1}\left(\Omega ; \mathbb{R}^{m}\right) \times$ $L^{1}\left(\Omega ; \mathbb{R}^{d}\right)$,

$$
\overline{F_{0}}(v, u ; \Omega) \leq \liminf _{n \rightarrow \infty} F\left(v_{n}, u_{n} ; \Omega\right),
$$

where $\bar{F}_{0}$ is given by (1.12).

Proof. Let $(v, u) \in S B V_{0}\left(\Omega ; \mathbb{R}^{m}\right) \times B V\left(\Omega ; \mathbb{R}^{d}\right)$. Without loss of generality, we may assume that for every $\left\{\left(v_{n}, u_{n}\right)\right\} \subset S B V_{0}\left(\Omega ; \mathbb{R}^{m}\right) \times B V\left(\Omega ; \mathbb{R}^{d}\right)$ converging to $(v, u)$ in $L^{1}\left(\Omega ; \mathbb{R}^{m}\right) \times L^{1}\left(\Omega ; \mathbb{R}^{d}\right)$,

$$
\begin{aligned}
& \liminf _{n \rightarrow \infty}\left(\int_{\Omega} f\left(v_{n}, \nabla u_{n}\right) d x+\int_{J_{v_{n}} \cap \Omega} g\left(v_{n}^{+}, v_{n}^{-}, \nu_{v_{n}}\right) d \mathcal{H}^{N-1}\right) \\
& =\lim _{n \rightarrow \infty}\left(\int_{\Omega} f\left(v_{n}, \nabla u_{n}\right) d x+\int_{J_{v_{n}} \cap \Omega} g\left(v_{n}^{+}, v_{n}^{-}, \nu_{v_{n}}\right) d \mathcal{H}^{N-1}\right)<+\infty .
\end{aligned}
$$

For every Borel set $B \subset \Omega$ define

$$
\mu_{n}(B):=\int_{B} f\left(v_{n}, \nabla u_{n}\right) d x+\int_{J_{v_{n}} \cap B} g\left(v_{n}^{+}, v_{n}^{-}, \nu_{v_{n}}\right) d \mathcal{H}^{N-1} .
$$

Since $\left\{\mu_{n}\right\}$ is a sequence of nonnegative Radon measures, uniformly bounded in the space of measures, we can extract a subsequence, still denoted by $\left\{\mu_{n}\right\}$, weakly $*$ converging in the sense of measures to some Radon measure $\mu$. Using Radon-Nikodým theorem we can decompose $\mu$ as a sum of four mutually singular nonnegative measures, namely

$$
\mu=\mu_{a} \mathcal{L}^{N}+\mu_{c}\left|D^{c} u\right|+\mu_{j} \mathcal{H}^{N-1}\left\lfloor J_{(v, u)}+\mu_{s},\right.
$$

where we have been considering $(v, u)$ as a unique field in $B V\left(\Omega ; \mathbb{R}^{m+d}\right)$ and we have been exploiting the fact that $D^{c}(v, u)=\left(\underline{0}, D^{c} u\right)$ (cf. Remark 2.11). By Besicovitch derivation theorem

$$
\begin{aligned}
& \mu_{a}\left(x_{0}\right)=\lim _{\varepsilon \rightarrow 0^{+}} \frac{\mu\left(B\left(x_{0}, \varepsilon\right)\right)}{\mathcal{L}^{N}\left(B\left(x_{0}, \varepsilon\right)\right)}<+\infty, \text { for } \mathcal{L}^{N}-\text { a.e. } x_{0} \in \Omega, \\
& \mu_{j}\left(x_{0}\right)=\lim _{\varepsilon \rightarrow 0^{+}} \frac{\mu\left(Q_{\nu}\left(x_{0}, \varepsilon\right)\right)}{\mathcal{H}^{N-1}\left(Q_{\nu}\left(x_{0}, \varepsilon\right) \cap J_{(v, u)}\right)}<+\infty, \text { for } \mathcal{H}^{N-1}-\text { a.e. } x_{0} \in J_{(v, u)} \cap \Omega, \\
& \mu_{c}\left(x_{0}\right)=\lim _{\varepsilon \rightarrow 0^{+}} \frac{\mu\left(Q\left(x_{0}, \varepsilon\right)\right)}{|D u|\left(Q\left(x_{0}, \varepsilon\right)\right)}<+\infty, \text { for }\left|D^{c} u\right| \text { - a.e. } x_{0} \in \Omega .
\end{aligned}
$$

We claim that

$$
\begin{gathered}
\mu_{a}\left(x_{0}\right) \geq Q f\left(v\left(x_{0}\right), \nabla u\left(x_{0}\right)\right), \text { for } \mathcal{L}^{N}-\text { a.e. } x_{0} \in \Omega, \\
\mu_{j}\left(x_{0}\right) \geq K_{3}\left(v^{+}\left(x_{0}\right), v^{-}\left(x_{0}\right), u^{+}\left(x_{0}\right), u^{-}\left(x_{0}\right), \nu_{(v, u)}\right), \text { for } \mathcal{H}^{N-1}-\text { a.e. } x_{0} \in J_{(v, u)} \cap \Omega, \\
\mu_{c}\left(x_{0}\right) \geq(Q f)^{\infty}\left(v\left(x_{0}\right), \frac{d D^{c} u}{d\left|D^{c} u\right|}\left(x_{0}\right)\right) \text { for }\left|D^{c} u\right|-\text { a.e. } x_{0} \in \Omega,
\end{gathered}
$$

where $Q f$ is the density introduced in (3.2), $Q f^{\infty}$ is its recession function as in (1.10) and $K_{3}$ is given by (1.13). If (4.4) - (4.6) hold then (4.1) follows immediately. Indeed, since $\mu_{n} \stackrel{*}{\rightarrow} \mu$ in the sense of measures

$$
\begin{aligned}
& \liminf _{n \rightarrow \infty}\left(\int_{\Omega} f\left(v_{n}, \nabla u_{n}\right) d x+\int_{J_{v_{n}} \cap \Omega} g\left(v_{n}^{+}, v_{n}^{-}, \nu_{v_{n}}\right) d \mathcal{H}^{N-1}\right) \\
& \geq \liminf _{n \rightarrow \infty} \mu_{n}(\Omega) \geq \mu(\Omega) \geq \int_{\Omega} \mu_{a} d x+\int_{J_{(v, u)}} \mu_{j} d \mathcal{H}^{N-1}+\int_{\Omega} \mu_{c} d\left|D^{c} u\right| \\
& \geq \int_{\Omega} Q f(v(x), \nabla u(x)) d x+\int_{J_{u} \cap \Omega} K_{3}\left(v^{+}(x), v^{-}(x), u^{+}(x), u^{-}(x), \nu_{(v, u)}\right) d \mathcal{H}^{N-1} \\
& +\int_{\Omega}(Q f)^{\infty}\left(v(x), \frac{d D^{c} u}{d\left|D^{c} u\right|}(x)\right) d\left|D^{c} u\right|
\end{aligned}
$$


where we have used the fact that $\mu_{s}$ is nonnegative.

We prove (4.4) - (4.6) using the blow-up method introduced in [24].

Step 1. Let $x_{0} \in \Omega$ be a Lebesgue point for $\nabla u$ and $v$, such that $x_{0} \notin J_{(v, u)}$, (2.1) applied to $u$, and (4.3) hold.

We observe that

$$
\begin{aligned}
& \liminf _{n \rightarrow \infty}\left(\int_{\Omega} f\left(v_{n}, \nabla u_{n}\right) d x+\int_{J_{v_{n}} \cap \Omega} g\left(v_{n}^{+}, v_{n}^{-}, \nu_{v_{n}}\right) d \mathcal{H}^{N-1}\right) \\
& \geq \liminf _{n \rightarrow \infty} \int_{\Omega} f\left(v_{n}, \nabla u_{n}\right) d x \geq \liminf _{n \rightarrow \infty} \int_{\Omega} Q f\left(v_{n}, \nabla u_{n}\right) d x .
\end{aligned}
$$

Note that by Proposition 3.1 $Q f$ satisfies $\left(F_{1}\right)-\left(F_{3}\right)$. By Proposition 3.7 we may assume that $\left\{\left(v_{n}, u_{n}\right)\right\} \subset$ $C_{0}^{\infty}\left(\mathbb{R}^{N} ; \mathbb{R}^{m}\right) \times C_{0}^{\infty}\left(\mathbb{R}^{N} ; \mathbb{R}^{d}\right)$ and applying [25, formula (2.10) in Theorem 2.19], to the functional $G:(v, u) \in$ $W^{1,1}\left(\Omega ; \mathbb{R}^{m+d}\right) \rightarrow \int_{\Omega} Q f(v, \nabla u) d x$ we obtain (4.4).

Step 2. Now we prove (4.5) .

Remind that $J_{(v, u)}=J_{v} \cup J_{u}$ and $\nu_{(v, u)}=\nu_{v}$ for every $(v, u) \in S B V_{0}\left(\Omega ; \mathbb{R}^{m}\right) \times W^{1,1}\left(\Omega ; \mathbb{R}^{d}\right)$. By Lemma 2.7. Proposition 2.6 ii) and Theorem 2.1 we may fix $x_{0} \in J_{(v, u)} \cap \Omega$ such that

$$
\begin{aligned}
& \lim _{\varepsilon \rightarrow 0^{+}} \frac{1}{\varepsilon^{N-1}} \int_{J_{(v, u)} \cap Q_{\nu}\left(x_{0}, \varepsilon\right)}\left(\left|v^{+}(x)-v^{-}\left(x_{0}\right)\right|+\left|u^{+}(x)-u^{-}\left(x_{0}\right)\right|\right) d \mathcal{H}^{N-1} \\
& =\left|v^{+}\left(x_{0}\right)-v^{-}\left(x_{0}\right)\right|+\left|u^{+}\left(x_{0}\right)-u^{-}\left(x_{0}\right)\right|, \\
& \quad \lim _{\varepsilon \rightarrow 0^{+}} \frac{1}{\varepsilon^{N}} \int_{\left\{x \in Q_{\nu}\left(x_{0}, \varepsilon\right):\left(x-x_{0}\right) \cdot \nu(x)>0\right\}}\left|v(x)-v^{+}\left(x_{0}\right)\right|^{\frac{N}{N-1}} d x \\
& \quad+\lim _{\varepsilon \rightarrow 0^{+}} \frac{1}{\varepsilon^{N}} \int_{\left\{x \in Q_{\nu}\left(x_{0}, \varepsilon\right):\left(x-x_{0}\right) \cdot \nu(x)>0\right\}}\left|u(x)-u^{+}\left(x_{0}\right)\right|^{\frac{N}{N-1}} d x=0, \\
& \quad \lim _{\varepsilon \rightarrow 0^{+}} \frac{1}{\varepsilon^{N}} \int_{\left\{x \in Q_{\nu}\left(x_{0}, \varepsilon\right):\left(x-x_{0}\right) \cdot \nu(x)<0\right\}}\left|v(x)-v^{-}\left(x_{0}\right)\right|^{\frac{N}{N-1}} d x \\
& \quad+\lim _{\varepsilon \rightarrow 0^{+}} \frac{1}{\varepsilon^{N}} \int_{\left\{x \in Q_{\nu}\left(x_{0}, \varepsilon\right):\left(x-x_{0}\right) \cdot \nu(x)<0\right\}}\left|u(x)-u^{-}\left(x_{0}\right)\right|^{\frac{N}{N-1}} d x=0, \\
& \quad \mu_{j}\left(x_{0}\right)=\lim _{\varepsilon \rightarrow 0^{+}} \frac{\mu\left(x_{0}+\varepsilon Q_{\nu\left(x_{0}\right)}\right)}{\mathcal{H}^{N-1}\left\lfloor J_{(v, u)}\left(x_{0}+\varepsilon Q_{\nu\left(x_{0}\right)}\right)\right.} \text { exists and it is finite. }
\end{aligned}
$$

For simplicity of notation we write $Q:=Q_{\nu\left(x_{0}\right)}$. Then by (4.10),

$$
\mu_{j}\left(x_{0}\right)=\lim _{\varepsilon \rightarrow 0^{+}} \frac{1}{\varepsilon^{N-1}} \int_{x_{0}+\varepsilon Q} d \mu(x) .
$$

Without loss of generality, we may choose $\varepsilon>0$ such that $\mu\left(\partial\left(x_{0}+\varepsilon Q\right)\right)=0$. Since $Q f \leq f$, we have

$$
\begin{aligned}
\mu_{j}\left(x_{0}\right) & \geq \lim _{\varepsilon \rightarrow 0^{+}} \lim _{n \rightarrow \infty} \frac{1}{\varepsilon^{N-1}}\left(\int_{x_{0}+\varepsilon Q} Q f\left(v_{n}(x), \nabla u_{n}(x)\right) d x+\int_{J_{v_{n}}} g\left(v_{n}^{+}, v_{n}^{-}, \nu_{v_{n}}\right) d \mathcal{H}^{N-1}\right) \\
& =\lim _{\varepsilon \rightarrow 0^{+}} \lim _{n \rightarrow \infty} \varepsilon \int_{Q} Q f\left(v_{n}\left(x_{0}+\varepsilon y\right), \nabla u_{n}\left(x_{0}+\varepsilon y\right)\right) d y \\
& +\int_{Q \cap J\left(v_{n}, u_{n}\right)-\frac{x_{0}}{\varepsilon}} g\left(v_{n}^{+}\left(x_{0}+\varepsilon y\right), v_{n}^{-}\left(x_{0}+\varepsilon y\right), \nu_{\left(v_{n}, u_{n}\right)}\left(x_{0}+\varepsilon y\right)\right) d \mathcal{H}^{N-1}(y) .
\end{aligned}
$$

Define

$$
v_{n, \varepsilon}(y):=v_{n}\left(x_{0}+\varepsilon y\right), u_{n, \varepsilon}(y):=u_{n}\left(x_{0}+\varepsilon y\right), \nu_{n, \varepsilon}(y):=\nu_{\left(v_{n}, u_{n}\right)}\left(x_{0}+\varepsilon y\right)
$$


and

$$
v_{0}(y):=\left\{\begin{array}{ll}
v^{+}\left(x_{0}\right) & \text { if } y \cdot \nu\left(x_{0}\right)>0, \\
v^{-}\left(x_{0}\right) & \text { if } y \cdot \nu\left(x_{0}\right)<0,
\end{array} \quad u_{0}(y):= \begin{cases}u^{+}\left(x_{0}\right) & \text { if } y \cdot \nu\left(x_{0}\right)>0 \\
u^{-}\left(x_{0}\right) & \text { if } y \cdot \nu\left(x_{0}\right)<0\end{cases}\right.
$$

Since $\left(v_{n}, u_{n}\right) \rightarrow(v, u)$ in $L^{1}\left(\Omega ; \mathbb{R}^{m+d}\right)$, by (4.8) and (4.9) one obtains

$$
\begin{aligned}
& \lim _{\varepsilon \rightarrow 0^{+}} \lim _{n \rightarrow \infty} \int_{Q}\left|v_{n, \varepsilon}(y)-v_{0}(y)\right| d y=\lim _{\varepsilon \rightarrow 0^{+}} \frac{1}{\varepsilon^{N}}\left(\int_{\left\{x \in x_{0}+\varepsilon \partial Q:\left(x-x_{0}\right) \cdot \nu\left(x_{0}\right)>0\right\}}\left|v(x)-v^{+}\left(x_{0}\right)\right| d x\right. \\
& \left.+\int_{\left\{x \in x_{0}+\varepsilon \partial Q:\left(x-x_{0}\right) \cdot \nu\left(x_{0}\right)<0\right\}}\left|v(x)-v^{-}\left(x_{0}\right)\right| d x\right)=0
\end{aligned}
$$

and

$$
\begin{aligned}
& \lim _{\varepsilon \rightarrow 0^{+}} \lim _{n \rightarrow \infty} \int_{Q}\left|u_{n, \varepsilon}(y)-u_{0}(y)\right| d y=\lim _{\varepsilon \rightarrow 0^{+}} \frac{1}{\varepsilon^{N}}\left(\int_{\left\{x \in x_{0}+\varepsilon \partial Q:\left(x-x_{0}\right) \cdot \nu\left(x_{0}\right)>0\right\}}\left|u(x)-u^{+}\left(x_{0}\right)\right| d x\right. \\
& \left.+\int_{\left\{x \in x_{0}+\varepsilon \partial Q:\left(x-x_{0}\right) \cdot \nu\left(x_{0}\right)<0\right\}}\left|u(x)-u^{-}\left(x_{0}\right)\right| d x\right)=0 .
\end{aligned}
$$

Thus

$$
\begin{aligned}
\mu_{j}\left(x_{0}\right) & \geq \lim _{\varepsilon \rightarrow 0^{+}} \lim _{n \rightarrow \infty}\left(\int_{Q} Q f^{\infty}\left(v_{n, \varepsilon}(y), \nabla u_{n, \varepsilon}(y)\right) d y+\int_{Q \cap J\left(v_{n, \varepsilon}, u_{n_{\varepsilon}}\right)} g\left(v_{n, \varepsilon}^{+}, v_{n, \varepsilon}^{-}, \nu_{v_{n, \varepsilon}}\right) d \mathcal{H}^{N-1}(y)\right. \\
& \left.+\int_{Q}\left(\varepsilon Q f\left(v_{n, \varepsilon}(y), \frac{1}{\varepsilon} \nabla u_{n, \varepsilon}(y)\right)-Q f^{\infty}\left(v_{n, \varepsilon}, \nabla u_{n, \varepsilon}\right)\right) d y\right) .
\end{aligned}
$$

Exploiting $(v)$ in Remark 3.2 we can argue as in the estimates [25, (3.3)-(3.5)], thus obtaining

$$
\mu_{j}\left(x_{0}\right) \geq \liminf _{\varepsilon \rightarrow 0^{+}} \liminf _{n \rightarrow \infty}\left(\int_{Q} Q f^{\infty}\left(v_{n, \varepsilon}(y), \nabla u_{n, \varepsilon}(y)\right) d y+\int_{Q \cap J\left(v_{n, \varepsilon}, u_{n, \varepsilon}\right)} g\left(v_{n, \varepsilon}^{+}, v_{n, \varepsilon}^{-}, \nu_{v_{n, \varepsilon}}\right) d \mathcal{H}^{N-1}(y)\right) .
$$

Since $\left(v_{n, \varepsilon}, u_{n, \varepsilon}\right) \rightarrow\left(v_{0}, u_{0}\right)$ in $L^{1}\left(Q ; \mathbb{R}^{m+d}\right)$ as $n \rightarrow \infty$ and $\varepsilon \rightarrow 0^{+}$, by a standard diagonalization argument, as in [12, Theorem 4.1 Steps 2 and 3], we obtain a sequence $\left(\bar{v}_{k}, \bar{u}_{k}\right)$ converging to $\left(v_{0}, u_{0}\right)$ in $L^{1}\left(Q ; \mathbb{R}^{m+d}\right)$ as $k \rightarrow \infty$ such that

$$
\mu_{j}\left(x_{0}\right) \geq \lim _{k \rightarrow \infty}\left(\int_{Q} Q f^{\infty}\left(\bar{v}_{k}(y), \nabla \bar{u}_{k}(y)\right) d y+\int_{Q \cap J_{\left(v_{k}, w_{k}\right)}} g\left(\bar{v}_{k}^{+}, \bar{v}_{k}^{-}, \nu_{\bar{v}_{k}}\right) d \mathcal{H}^{N-1}(y)\right) .
$$

Applying Lemma 3.8 with $Q f$ replaced by $Q f^{\infty}$ and using $(v)$ in Remark 3.2 we may find $\left\{\left(\zeta_{k}, \xi_{k}\right)\right\} \in$ $\mathcal{A}_{3}\left(v^{+}\left(x_{0}\right), v^{-}\left(x_{0}\right), u^{+}\left(x_{0}\right), u^{-}\left(x_{0}\right), \nu\left(x_{0}\right)\right)$ such that

$$
\begin{aligned}
& \mu_{j}\left(x_{0}\right) \geq \lim _{k \rightarrow \infty}\left(\int_{Q} Q f^{\infty}\left(\zeta_{k}, \nabla \xi_{k}\right) d x+\int_{Q \cap J_{\left(\zeta_{k}, \xi_{k}\right)}} g\left(\zeta_{k}^{+}, \zeta_{k}^{-}, \nu_{\zeta_{k}}\right) d \mathcal{H}^{N-1}\right) \\
& \geq K_{3}\left(v^{+}\left(x_{0}\right), v^{-}\left(x_{0}\right), u^{+}\left(x_{0}\right), u^{-}\left(x_{0}\right), \nu\left(x_{0}\right)\right) .
\end{aligned}
$$

Step 3. Here we show (4.6).

Let $(v, u) \in S B V_{0}\left(\Omega ; \mathbb{R}^{m}\right) \times B V\left(\Omega ; \mathbb{R}^{d}\right)$, note, as already emphasized in Remark 2.11, that $\left|D^{c}(v, u)\right|=\left|D^{c} u\right|$. For $\left|D^{c} u\right|$-a.e. $x_{0} \in \Omega$ we have

$$
\lim _{\varepsilon \rightarrow 0^{+}} \frac{|D(v, u)|\left(Q\left(x_{0}, \varepsilon\right)\right)}{\left|D^{c}(v, u)\right|\left(Q\left(x_{0}, \varepsilon\right)\right)}=\lim _{\varepsilon \rightarrow 0^{+}} \frac{|D(v, u)|\left(Q\left(x_{0}, \varepsilon\right)\right)}{\left|D^{c} u\right|\left(Q\left(x_{0}, \varepsilon\right)\right)}=1 .
$$

And so by Theorems 2.4. iii) and 2.11 in [25], and by Theorem[2.1] for $\left|D^{c} u\right|$-a.e. $x_{0} \in \Omega$ the following hold

$$
\mu_{c}\left(x_{0}\right)=\lim _{\varepsilon \rightarrow 0^{+}} \frac{\mu\left(Q\left(x_{0}, \varepsilon\right)\right)}{|D u|\left(Q\left(x_{0}, \varepsilon\right)\right)},
$$




$$
\lim _{\varepsilon \rightarrow 0^{+}} \frac{1}{\varepsilon^{N}} \int_{Q\left(x_{0}, \varepsilon\right)}\left(\left|u(x)-u\left(x_{0}\right)\right|+\left|v(x)-v\left(x_{0}\right)\right|\right) d x=0,
$$

for $\mathcal{H}^{N-1}-x_{0} \in \Omega \backslash J_{(v, u)}$,

$$
A\left(x_{0}\right)=\lim _{\varepsilon \rightarrow 0^{+}} \frac{(D(v, u))\left(Q\left(x_{0}, \varepsilon\right)\right)}{|D(v, u)|\left(Q\left(x_{0}, \varepsilon\right)\right)}, \quad\left\|A\left(x_{0}\right)\right\|=1, \quad A\left(x_{0}\right)=a \otimes \nu,
$$

with $a \in \mathbb{R}^{d}$ and $\nu \in S^{N-1}$,

$$
\begin{aligned}
& \lim _{\varepsilon \rightarrow 0^{+}} \frac{|D(v, u)|\left(Q\left(x_{0}, \varepsilon\right)\right)}{\varepsilon^{N-1}}=\lim _{\varepsilon \rightarrow 0^{+}} \frac{|D u|\left(Q\left(x_{0}, \varepsilon\right)\right)}{\varepsilon^{N-1}}=0, \\
& \lim _{\varepsilon \rightarrow 0^{+}} \frac{|D(v, u)|\left(Q\left(x_{0}, \varepsilon\right)\right)}{\varepsilon^{N}}=\lim _{\varepsilon \rightarrow 0^{+}} \frac{|D u|\left(Q\left(x_{0}, \varepsilon\right)\right)}{\varepsilon^{N}}=\infty .
\end{aligned}
$$

Arguing as in the end of Step 1, by Proposition 3.7 (ii), we may assume that $\left\{\left(\widetilde{v}_{n}, \widetilde{u}_{n}\right)\right\} \subset C_{0}^{\infty}\left(\mathbb{R}^{N} ; \mathbb{R}^{m+d}\right)$. Applying [25, formula (2.12) in Theorem 2.19], to the functional $G:(v, u) \in W^{1,1}\left(\Omega ; \mathbb{R}^{m+d}\right) \rightarrow \int_{\Omega} Q f(v, \nabla u) d x$ we obtain for $\left|D^{c}(v, u)\right|-$ a.e. $x_{0} \in \Omega$

$$
\mu_{c}\left(x_{0}\right) \geq(Q f)^{\infty}\left(v\left(x_{0}\right), \frac{d D^{c} u}{d\left|D^{c} u\right|}\left(x_{0}\right)\right)
$$

and that concludes the proof.

\section{$5 \quad$ Upper bound}

This section is devoted to prove that $\mathcal{F} \leq \bar{F}_{0}$.

Theorem 5.1 Let $\Omega \subset \mathbb{R}^{N}$ be a bounded open set, let $f: \mathbb{R}^{d} \times \mathbb{R}^{m} \rightarrow[0,+\infty)$, be a function satisfying $\left(F_{1}\right)$ - $\left(F_{4}\right)$, and let $g: \mathbb{R}^{m} \times \mathbb{R}^{m} \times S^{N-1} \rightarrow\left[0,+\infty\left[\right.\right.$ be a function satisfying $\left(G_{1}\right)$ - $\left(G_{3}\right)$.

Then for every $(v, u) \in S B V_{0}\left(\Omega ; \mathbb{R}^{m}\right) \times B V\left(\Omega ; \mathbb{R}^{d}\right)$, for every $A \in \mathcal{A}(\Omega)$, there exist sequences $\left\{v_{n}\right\} \subset$ $S B V_{0}\left(\Omega ; \mathbb{R}^{m}\right),\left\{u_{n}\right\} \subset W^{1,1}\left(\Omega ; \mathbb{R}^{d}\right)$ such that $v_{n} \rightarrow v$ in $L^{1}\left(\Omega ; \mathbb{R}^{m}\right), u_{n} \rightarrow u$ in $L^{1}\left(\Omega ; \mathbb{R}^{d}\right)$, and

$$
\liminf _{n \rightarrow \infty} F\left(v_{n}, u_{n} ; A\right) \leq \bar{F}_{0}(v, u ; A) .
$$

Before proving the upper bound we recall our strategy, which was first proposed in [9] and further developped in [25]. Namely, first we will show that $\mathcal{F}(v, u ; \cdot)$ is a variational functional with respect to the $L^{1}$ topology and

$$
\mathcal{F}(v, u ; \cdot) \leq \mathcal{L}^{N}+|D v|+|D u|+\mathcal{H}^{N-1}\left\lfloor J_{v} .\right.
$$

Next by Besicovitch differentiation Theorem, a blow-up argument will provide an upper bound estimate in terms of $\bar{F}_{0}$, first for bulk and Cantor parts, then also for the jump part, when the target functions $(v, u)$ are bounded. Finally the same approximation as in [9, Theorem 4.9], will give the estimate for every $(v, u) \in S B V_{0}\left(\Omega ; \mathbb{R}^{m}\right) \times B V\left(\Omega ; \mathbb{R}^{d}\right)$.

We recall that $\mathcal{F}(v, u ; \cdot)$ is said to be a variational functional with respect to the $L^{1}$ topology if

(i) $\mathcal{F}(\cdot, \cdot ; A)$ is local, i.e., $\mathcal{F}(v, u ; A)=\mathcal{F}\left(v^{\prime}, u^{\prime} ; A\right)$ for every $v, v^{\prime} \in S B V_{0}\left(A ; \mathbb{R}^{m}\right), u, u^{\prime} \in B V\left(A ; \mathbb{R}^{d}\right)$ satisfying $u=u^{\prime}, v=v^{\prime}$ a.e. in $A$.

(ii) $\mathcal{F}(\cdot, \cdot ; A)$ is sequentially lower semicontinuous, i.e., if $v_{n}, v \in B V\left(A ; \mathbb{R}^{m}\right), u_{n}, u \in B V\left(A ; \mathbb{R}^{d}\right)$ and $v_{n} \rightarrow v$ in $L^{1}\left(A ; \mathbb{R}^{m}\right), u_{n} \rightarrow u$ in $L^{1}\left(A ; \mathbb{R}^{d}\right)$ then $\mathcal{F}(v, u ; A) \leq \liminf _{n \rightarrow \infty} \mathcal{F}\left(v_{n}, u_{n} ; A\right)$.

(iii) $\mathcal{F}(\cdot, \cdot ; A)$ is the trace on $\{A \subset \Omega: A$ is open $\}$ of a Borel measure on $\mathcal{B}(\Omega)$ the family of all Borel subsets of $\Omega$.

Since the lower semicontinuity and the locality of $\mathcal{F}(\cdot, \cdot ; A)$ follow by its definition, it remains to prove (iii). This is the target of the following lemma, where (iii) will be obtained via a refinement of De GiorgiLetta criterion, cf. [20, Corollary 5.2]. 
Lemma 5.2 Let $\Omega \subset \mathbb{R}^{N}$ be an open bounded set with Lipschitz boundary and let $f$ and $g$ be as in Theorem 5.1. For every $(v, u) \in S B V_{0}\left(\Omega ; \mathbb{R}^{m}\right) \times B V\left(\Omega ; \mathbb{R}^{d}\right)$, the set function $\mathcal{F}(v, u ; \cdot)$ in (1.11) is the trace of a Radon measure absolutely continuous with respect to $\mathcal{L}^{N}+|D v|+|D u|+\mathcal{H}^{N-1}\left\lfloor J_{v}\right.$.

Proof. An argument very similar to [14, Lemma 2.6 and Remark 2.7] and [10, Lemma 4.7] entails

$$
\mathcal{F}(v, u ; A) \leq C\left(\mathcal{L}^{N}(A)+|D v|(A)+|D u|(A)+\mathcal{H}^{N-1}\left\lfloor J_{v}(A)\right) .\right.
$$

By [20, Corollary 5.2] to obtain (iii) it suffices to prove that

$$
\mathcal{F}(v, u ; A) \leq \mathcal{F}(v, u ; B)+\mathcal{F}(v, u ; A \backslash \bar{U})
$$

for all $A, U, B \in \mathcal{A}(\Omega)$ with $U \subset \subset B \subset \subset A, u \in B V\left(\Omega ; \mathbb{R}^{d}\right)$ and $v \in S B V_{0}\left(\Omega ; \mathbb{R}^{m}\right)$.

We start by assuming that $v \in S B V_{0}\left(\Omega ; \mathbb{R}^{m}\right) \cap L^{\infty}\left(\Omega ; \mathbb{R}^{m}\right)$.

Fix $\eta>0$ and find $\left\{w_{n}\right\} \subset W^{1,1}\left((A \backslash \bar{U}) ; \mathbb{R}^{d}\right),\left\{v_{n}\right\} \subset S B V_{0}\left(A \backslash \bar{U} ; \mathbb{R}^{m}\right) \cap L^{\infty}\left(A \backslash \bar{U} ; \mathbb{R}^{m}\right)$ (cf. Remark 3.9) such that $w_{n} \rightarrow u$ in $L^{1}\left((A \backslash \bar{U}) ; \mathbb{R}^{d}\right)$ and $v_{n} \rightarrow v$ in $L^{1}\left((A \backslash \bar{U}) ; \mathbb{R}^{m}\right)$ and

$$
\limsup _{n \rightarrow \infty} \int_{A \backslash \bar{U}} f\left(v_{n}, \nabla w_{n}\right) d x+\int_{A \backslash \bar{U} \cap J_{v_{n}}} g\left(v_{n}^{+} . v_{n}^{-}, \nu_{v_{n}}\right) d \mathcal{H}^{N-1} \leq \mathcal{F}(v, u ; A \backslash \bar{U})+\eta .
$$

Extract a subsequence still denoted by $n$ such that the above upper limit is a limit.

Let $B_{0}$ be an open subset of $\Omega$ with Lipschitz boundary such that $U \subset \subset B_{0} \subset \subset B$. Then there exist $\left\{u_{n}\right\} \subset W^{1,1}\left(B_{0} ; \mathbb{R}^{d}\right)$ and $\left\{\bar{v}_{n}\right\} \subset S B V_{0}\left(B_{0} ; \mathbb{R}^{m}\right) \cap L^{\infty}\left(B_{0} ; \mathbb{R}^{m}\right)$ (cf. (i) in Remark [3.9) such that $u_{n} \rightarrow u$ in $L^{1}\left(B_{0} ; \mathbb{R}^{d}\right)$ and $\bar{v}_{n} \rightarrow v$ in $L^{1}\left(B_{0} ; \mathbb{R}^{m}\right)$ and

$$
\mathcal{F}\left(v, u ; B_{0}\right)=\lim _{n \rightarrow \infty}\left(\int_{B_{0}} f\left(\bar{v}_{n}, \nabla u_{n}\right) d x+\int_{J_{\bar{v}_{n}} \cap B_{0}} g\left(\bar{v}_{n}{ }^{+}, \bar{v}_{n}{ }^{-}, \nu_{\bar{v}_{n}}\right) d \mathcal{H}^{N-1}\right) .
$$

For every $(\bar{v}, w) \in S B V_{0}\left(A ; \mathbb{R}^{m}\right) \cap L^{\infty}\left(A ; \mathbb{R}^{m}\right) \times W^{1,1}\left(A ; \mathbb{R}^{d}\right)$, consider $\mathcal{G}_{n}(\bar{v}, w ; A):=\int_{A}(1+|\nabla w|) d x$ $+(1+[\bar{v}]) \mathcal{H}^{N-1}\left\lfloor\left(J_{\bar{v}} \cap A\right)\right.$.

Due to the coercivity (1.1), we may extract a bounded subsequence not relabelled, from the sequence of measures $\nu_{n}:=\mathcal{G}_{n}\left(v_{n}, w_{n} ; \cdot\right)+\mathcal{G}_{n}\left(\bar{v}_{n}, u_{n} ; \cdot\right)$ restricted to $B_{0} \backslash \bar{U}$, converging in the sense of distributions to some Radon measure $\nu$, defined on $B_{0} \backslash \bar{U}$. Analogously, for every $w \in S B V_{0}\left(A ; \mathbb{R}^{m}\right) \cap L^{\infty}\left(A ; \mathbb{R}^{m}\right)$ we could define a sequence of measures $\mathcal{H}_{n}(w ; E):=\int_{J_{w} \cap E} d \mathcal{H}^{N-1}$.

For every $t>0$, let $B_{t}:=\left\{x \in B_{0} \mid \operatorname{dist}\left(x, \partial B_{0}\right)>t\right\}$. Define, for $0<\delta<\eta$, the subsets $L_{\delta}:=B_{\eta-2 \delta} \backslash \overline{B_{\eta+\delta}}$. Consider a smooth cut-off function $\varphi_{\delta} \in C_{0}^{\infty}\left(B_{\eta-\delta} ;[0,1]\right)$ such that $\varphi_{\delta}(x)=1$ on $B_{\eta}$. As the thickness of the strip is of order $\delta$, we have an upper bound of the form $\left\|\nabla \varphi_{\delta}\right\|_{L^{\infty}\left(B_{\eta-\delta}\right)} \leq \frac{C}{\delta}$.

Define $\bar{w}_{n}(x):=\varphi_{\delta}(x) u_{n}(x)+\left(1-\varphi_{\delta}(x)\right) w_{n}(x)$. Clearly, $\left\{\bar{w}_{n}\right\}$ converges to $u$ in $L^{1}(A)$ as $n \rightarrow \infty$, and

$$
\nabla \bar{w}_{n}=\varphi_{\delta} \nabla u_{n}+\left(1-\varphi_{\delta}\right) \nabla w_{n}+\nabla \varphi_{\delta} \otimes\left(u_{n}-w_{n}\right) .
$$

Arguing as in [5, Lemma 4.4], we may consider a sharp transition for the $S B V_{0}$ functions, namely let $\left\{v_{n}\right\}$ and $\left\{\bar{v}_{n}\right\}$ be as above, then for every $0<t<1$ we may define $\tilde{v}_{n}^{t}$ such that $\tilde{v}_{n}^{t} \rightarrow v$ in $L^{1}(A)$ as $n \rightarrow \infty$, and

$$
\tilde{v}_{n}^{t}(x):= \begin{cases}v_{n}(x) & \text { in }\left\{x: \varphi_{\delta}(x)<t\right\} \\ \bar{v}_{n}(x) & \text { in }\left\{x: \varphi_{\delta}(x) \geq t\right\}\end{cases}
$$

Clearly $\tilde{v}_{n}^{t}(x) \in\left\{v_{n}(x), \bar{v}_{n}(x)\right\}$ almost everywhere in $A$, and since $\mathcal{H}^{N-1}\left(J_{v_{n}}\right), \mathcal{H}^{N-1}\left(J_{\bar{v}_{n}}\right)<+\infty$ for all but at most countable $t \in] 0,1[$ it results that

$$
\mathcal{H}^{N-1}\left(J_{v_{n}} \cap\left\{x \in A: \varphi_{\delta}(x)=t\right\}\right)=\mathcal{H}^{N-1}\left(J_{\bar{v}_{n}} \cap\left\{x \in A: \varphi_{\delta}(x)=t\right\}\right)=0 .
$$

Moreover, using coarea formula (2.3) and the mean value theorem it is possible to find a $t$ for which the integral over the level set is comparable with the double integral with $t$ varying between 0 and 1 . Thus we have

$$
\int_{\partial^{*}\left\{\varphi_{\delta}<t\right\}} d \mathcal{H}^{N-1} \leq \frac{C}{\delta} \mathcal{L}^{N}\left(B_{\eta-\delta} \backslash B_{\eta}\right) \leq C .
$$


An analogous reasoning provides for the same $t$ that

$$
\int_{\partial^{*}\left\{\varphi_{\delta}<t\right\}}\left|\left[\tilde{v}_{n}^{t}\right]\right| d \mathcal{H}^{N-1} \leq \frac{C}{\delta} \int_{B_{\eta-\delta} \backslash B_{\eta}}\left|v_{n}(x)-\bar{v}_{n}(x)\right| d x .
$$

Thus, as for the $\left\{\mathcal{G}_{n}\right\}$ above, we may extract a bounded subsequence not relabelled, from the sequence of measures $\mathcal{H}_{n}\left(\tilde{v}_{n}^{t}, \cdot\right)$, restricted to $B_{0} \backslash \bar{U} \cap \partial^{*}\left\{\varphi_{\delta}<t\right\}$, converging in the sense of distributions to some Radon measure $\nu_{1}$, defined on $B_{0} \backslash \bar{U}$.

By (1.1) we have the estimate

$$
\begin{aligned}
& \int_{A} f\left(\tilde{v}_{n}^{t}, \nabla \bar{w}_{n}\right) d x+\int_{A \cap \bar{v}_{n}^{t}} g\left(\left(\tilde{v}_{n}^{t}\right)^{+},\left(\tilde{v}_{n}^{t}\right)^{-}, \nu_{\tilde{v}_{n}^{t}}\right) d \mathcal{H}^{N-1} \\
& \leq \int_{B_{\eta}} f\left(\bar{v}_{n}, \nabla u_{n}\right) d x+\int_{J_{\bar{v}_{n}} \cap B_{\eta}} g\left(\bar{v}_{n}{ }^{+}, \bar{v}_{n}{ }^{-}, \nu_{\bar{v}_{n}}\right) d \mathcal{H}^{N-1} \\
& +\int_{\left(A \backslash \overline{B_{\eta-\delta}}\right)} f\left(v_{n}, \nabla w_{n}\right) d x+\int_{J_{v_{n}} \cap\left(A \backslash \overline{B_{\eta-\delta}}\right)} g\left(v_{n}^{+}, v_{n}^{-}, \nu_{v_{n}}\right) d \mathcal{H}^{N-1} \\
& +C\left(\mathcal{G}_{n}\left(v_{n}, w_{n} ; L_{\delta}\right)+\mathcal{G}_{n}\left(\bar{v}_{n}, u_{n} ; L_{\delta}\right)\right)+\frac{1}{\delta} \int_{L_{\delta}}\left|w_{n}-u_{n}\right| d x+\int_{\partial^{*}\left\{\varphi_{\delta}<t\right\}}\left|\left[\tilde{v}_{n}^{t}\right]\right| d \mathcal{H}^{N-1}+\mathcal{H}_{n}\left(\tilde{v}_{n}^{t} ; L_{\delta} \cap \partial^{*}\left\{\varphi_{\delta}<t\right\}\right) \\
& \leq \int_{B_{0}} f\left(\bar{v}_{n}, \nabla u_{n}\right) d x+\int_{J_{\bar{v}_{n}} \cap B_{0}} g\left(\bar{v}_{n}{ }^{+}, \bar{v}_{n}{ }^{-}, \nu_{\bar{v}_{n}}\right) d \mathcal{H}^{N-1} \\
& +\int_{(A \backslash \bar{U})} f\left(v_{n}, \nabla w_{n}\right) d x+\int_{J_{v_{n}} \cap(A \backslash \bar{U})} g\left(v_{n}^{+}, v_{n}^{-}, \nu_{v_{n}}\right) d \mathcal{H}^{N-1} \\
& +C\left(\mathcal{G}_{n}\left(v_{n}, w_{n}, L_{\delta}\right)+\mathcal{G}_{n}\left(\bar{v}_{n}, u_{n}, L_{\delta}\right)\right)+\frac{1}{\delta} \int_{L_{\delta}}\left|w_{n}-u_{n}\right| d x+\int_{\partial^{*}\left\{\varphi_{\delta}<t\right\}}\left|\left[\tilde{v}_{n}^{t}\right]\right| d \mathcal{H}^{N-1}+\mathcal{H}_{n}\left(\tilde{v}_{n}^{t} ; L_{\delta} \cap \partial^{*}\left\{\varphi_{\delta}<t\right\}\right)
\end{aligned}
$$

Passing to the limit as $n \rightarrow \infty$, and applying (5.1), (5.2), (5.3) and the $L^{1}$ convergence of $\left\{v_{n}\right\}$ and $\left\{\bar{v}_{n}\right\}$ to $v$, it results that

$$
\begin{aligned}
\mathcal{F}(v, u ; A) & \leq \mathcal{F}\left(v, u ; B_{0}\right)+\mathcal{F}(v, u ; A \backslash \bar{U})+\eta+C \nu\left(\overline{L_{\delta}}\right)+C \nu_{1}\left(\overline{L_{\delta}}\right)+\limsup _{n \rightarrow \infty} \int_{\partial^{*}\left\{\varphi_{\delta}<t\right\}}\left|\left[\tilde{v}_{n}^{t}\right]\right| d \mathcal{H}^{N-1} \\
& \leq \mathcal{F}(v, u ; B)+\mathcal{F}(v, u ; A \backslash \bar{U})+\eta+C \nu\left(\overline{L_{\delta}}\right)+C \nu_{1}\left(\overline{L_{\delta}}\right) .
\end{aligned}
$$

Letting $\delta$ go to 0 we obtain

$$
\mathcal{F}(v, u ; A) \leq \mathcal{F}(v, u ; B)+\mathcal{F}(v, u ;(A \backslash \bar{U}))+\eta+C \nu\left(\partial B_{\eta}\right)+C \nu_{1}\left(\partial B_{\eta}\right) .
$$

It suffices to choose a subsequence $\left\{\eta_{i}\right\}$ such that $\eta_{i} \rightarrow 0^{+}$and $\nu\left(\partial B_{\eta_{i}}\right)=\nu_{1}\left(\partial B_{\eta_{i}}\right)=0$, to conclude the proof of subadditivity in the case $v \in S B V_{0} \cap L^{\infty}$.

In the general case, by virtue of Remark 3.9, we can argue as in the last part of Theorem 10 in [13.

Proof of Theorem 5.1. We assume first that $(v, u) \in\left(S B V_{0}\left(\Omega ; \mathbb{R}^{m}\right) \times B V\left(\Omega ; \mathbb{R}^{d}\right)\right) \cap L^{\infty}\left(\Omega ; \mathbb{R}^{m+d}\right)$.

Step 1. In order to prove the upper bound, we start by recalling that by Proposition 3.6 we can replace $Q f$ by $f$ in (1.11). First we deal with the bulk part.

Since the $\mathcal{F}(v, u ; \cdot)$ is a measure absolutely continuous with respect to $\mathcal{L}^{N}+|D u|+(1+[v]) \mathcal{H}^{N-1}\left\lfloor J_{v}\right.$ we claim that

$$
\frac{d \mathcal{F}(v, u ; \cdot)}{d \mathcal{L}^{N}}\left(x_{0}\right) \leq Q f\left(v\left(x_{0}\right), \nabla u\left(x_{0}\right)\right)
$$


for $\mathcal{L}^{N}$-a.e. $x_{0} \in \Omega$ where $x_{0}$ is a Lebesgue point of $v$ and $u$ such that

$$
\begin{aligned}
& \lim _{\varepsilon \rightarrow 0^{+}} \frac{1}{\varepsilon}\left\{\frac{1}{\varepsilon^{N}} \int_{B\left(x_{0}, \varepsilon\right)}\left|u(x)-u\left(x_{0}\right)-\nabla u\left(x_{0}\right)\left(x-x_{0}\right)\right|^{\frac{N}{N-1}} d x\right\}^{\frac{N-1}{N}}=0, \\
& \lim _{\varepsilon \rightarrow 0^{+}} \frac{1}{\varepsilon}\left\{\frac{1}{\varepsilon^{N}} \int_{B\left(x_{0}, \varepsilon\right)}\left|v(x)-v\left(x_{0}\right)\right|^{\frac{N}{N-1}} d x\right\}^{\frac{N-1}{N}}=0, \\
& \mu_{a}\left(x_{0}\right)=\lim _{\varepsilon \rightarrow 0^{+}} \frac{\mu\left(B\left(x_{0}, \varepsilon\right)\right)}{\mathcal{L}^{N}\left(B\left(x_{0}, \varepsilon\right)\right)}<\infty .
\end{aligned}
$$

Let $U:=(v, u)$. By (5.4) and Theorems 2.1 and 2.2 for $\mathcal{L}^{N}$-a.e. $x_{0} \in \Omega$ we have

$$
\begin{aligned}
& \lim _{\varepsilon \rightarrow 0^{+}} \frac{1}{\mathcal{L}^{N}\left(B\left(x_{0}, \varepsilon\right)\right)} \int_{B\left(x_{0}, \varepsilon\right)}\left|U(x)-U\left(x_{0}\right)\right|(1+|\nabla U(x)|) d x=0, \\
& \lim _{\varepsilon \rightarrow 0^{+}} \frac{\left|D_{s} U\right|\left(B\left(x_{0}, \varepsilon\right)\right)}{\mathcal{L}^{N}\left(B\left(x_{0}, \varepsilon\right)\right)}=0, \\
& \lim _{\varepsilon \rightarrow 0^{+}} \frac{|D U|\left(B\left(x_{0}, \varepsilon\right)\right)}{\mathcal{L}^{N}\left(B\left(x_{0}, \varepsilon\right)\right)} \text { exists and it is finite, } \\
& \lim _{\varepsilon \rightarrow 0^{+}} \frac{1}{\mathcal{L}^{N}\left(B\left(x_{0}, \varepsilon\right)\right)} \int_{B\left(x_{0}, \varepsilon\right)} Q f\left(v\left(x_{0}\right), \nabla u(x)\right) d x=Q f\left(v\left(x_{0}\right), \nabla u\left(x_{0}\right)\right), \\
& \frac{d \mathcal{F}(v, u ; \cdot)}{d \mathcal{L}^{N}}\left(x_{0}\right) \text { exists and it is finite. }
\end{aligned}
$$

We observe that the assumptions imposed on $f$ and Proposition 3.1 allow us to apply for every $v \in$ $S B V_{0}\left(\Omega ; \mathbb{R}^{m}\right)$ the Global Method (cf. [14, Theorem 4.1.4]) to the functional $u \in W^{1,1}\left(\Omega ; \mathbb{R}^{d}\right) \times \mathcal{A}(\Omega) \rightarrow$ $G(u ; A):=\int_{A} Q f(v(x), \nabla u(x)) d x$, thus obtaining an integral representation for the relaxed functional

$$
\mathcal{G}(u ; A)=\inf \left\{\liminf _{n \rightarrow \infty} G\left(u_{n} ; A\right): u_{n} \rightarrow u \text { in } L^{1}\left(A ; \mathbb{R}^{d}\right)\right\}
$$

for every $(u, A) \in B V\left(\Omega ; \mathbb{R}^{d}\right) \times \mathcal{A}(\Omega)$.

Recall that the growth condition $\left(G_{2}\right)$, the lower semicontinuity with respect to the $L^{1}$-topology of the functional $v \in S B V_{0}\left(\Omega ; \mathbb{R}^{m}\right) \mapsto\left((1+[v]) \mathcal{H}^{N-1}\left\lfloor\left(J_{v} \cap A\right)\right.\right.$ entails

$$
\mathcal{F}(v, u ; A) \leq \mathcal{G}(u ; A)+(1+[v]) \mathcal{H}^{N-1}\left\lfloor\left(J_{v} \cap A\right),\right.
$$

Differentiating with respect to $\mathcal{L}^{N}$ at $x_{0}$ and exploiting (5.4) and (5.5) we obtain that

$$
\frac{d \mathcal{F}((v, u) ; \cdot)}{d \mathcal{L}^{N}}\left(x_{0}\right) \leq f_{0}\left(x_{0}, \nabla u\left(x_{0}\right)\right),
$$

where for every $x_{0} \in \Omega$ and $\xi \in \mathbb{R}^{d}, f_{0}\left(x_{0}, \xi\right)$ is given as in [14, formula (4.1.5)], namely

$$
f_{0}\left(x_{0}, \xi\right):=\limsup _{\varepsilon \rightarrow 0^{+}} \inf _{\substack{z \in W^{1,1}\left(Q ; \mathbb{R}^{d}\right) \\ z(y)=\xi y \text { on } \partial Q}}\left\{\int_{Q} Q f\left(v\left(x_{0}+\varepsilon y\right), \nabla z(y)\right) d y\right\} .
$$

To conclude the proof we claim that $f_{0}\left(x_{0}, \xi\right) \leq Q f\left(v\left(x_{0}\right), \xi\right)$ for every $x_{0} \in \Omega$ satisfying (5.4) and (5.5) and $\xi \in \mathbb{R}^{d}$.

By virtue of Lemma 3.10 we have that

$$
\begin{aligned}
& \limsup _{\varepsilon \rightarrow 0^{+}} \inf _{\substack{z \in W^{1,1}\left(Q ; \mathbb{R}^{d}\right) \\
z(y)=\xi y \text { on } \partial Q}}\left\{\int_{Q} Q f\left(v\left(x_{0}+\varepsilon y\right), \nabla z(y)\right) d y\right\} \\
& \leq \inf _{\substack{z \in W^{1,1}\left(Q ; \mathbb{R}^{d}\right) \\
z(y)=\xi y \text { on } \partial Q}}\left\{\limsup _{\varepsilon \rightarrow 0^{+}} \int_{Q} Q f\left(v\left(x_{0}+\varepsilon y\right), \nabla z(y)\right) d y\right\} .
\end{aligned}
$$


Computing the limsup on the right hand side, we have

$$
\begin{aligned}
& \limsup _{\varepsilon \rightarrow 0^{+}} \int_{Q} Q f\left(v\left(x_{0}+\varepsilon y\right), \nabla z(y)\right) d y \\
& =\limsup _{\varepsilon \rightarrow 0^{+}}\left(\int_{Q} Q f\left(v\left(x_{0}+\varepsilon y\right), \nabla z(y)\right) d y-\int_{Q} Q f\left(v\left(x_{0}\right), \nabla z(y)\right) d y\right)+\int_{Q} Q f\left(v\left(x_{0}\right), \nabla z(y)\right) d y .
\end{aligned}
$$

Since $x_{0}$ is a Lebesgue point for $v$, and recalling that $v \in S B V_{0}\left(Q ; \mathbb{R}^{m}\right) \cap L^{\infty}\left(Q ; \mathbb{R}^{m}\right)$, by Lebesgue dominated convergence theorem and $\left(F_{3}\right)$ applied to $Q f$ (see Proposition 3.1), we have that

$$
\begin{aligned}
& \limsup _{\varepsilon \rightarrow 0^{+}}\left(\int_{Q} Q f\left(v\left(x_{0}+\varepsilon y\right), \nabla z(y)\right) d y-\int_{Q} Q f\left(v\left(x_{0}\right), \nabla z(y)\right) d y\right) \\
& \leq \limsup _{\varepsilon \rightarrow 0^{+}} \int_{Q} L\left|v\left(x_{0}+\varepsilon y\right)-v\left(x_{0}\right)\right|(1+|\nabla z(y)|) d y=0 .
\end{aligned}
$$

Hence

$$
\limsup _{\varepsilon \rightarrow 0^{+}} \int_{Q} Q f\left(v\left(x_{0}+\varepsilon y\right), \nabla z(y)\right) d y=\int_{Q} Q f\left(v\left(x_{0}\right), \nabla z(y)\right) d y .
$$

By the quasiconvexity of $Q f\left(v\left(x_{0}\right), \cdot\right)$, and (5.8) one obtains

$$
f_{0}\left(x_{0}, \xi\right) \leq Q f\left(v\left(x_{0}\right), \xi\right)
$$

which concludes the proof, when replacing $\xi$ by $\nabla u\left(x_{0}\right)$.

Step 2. We prove the upper bound for the Cantor part.

By Radon-Nikodým theorem we can write

$$
|D U|=\left|D^{c} u\right|+\sigma
$$

where $U:=(v, u) \in\left(S B V_{0}\left(\Omega ; \mathbb{R}^{m}\right) \times B V\left(\Omega ; \mathbb{R}^{d}\right)\right) \cap L^{\infty}\left(\Omega ; \mathbb{R}^{m+d}\right), \sigma$ and $\left|D^{c} u\right|$ are mutually singular Radon measures.

Observe that $U \equiv(v, u)$ is $\left|D^{c} u\right|$-measurable, $D v$ is singular with respect to $\left|D^{c} u\right|$ and by Theorems 2.1, 2.2, and [25, Theorem 2.11] for $\left|D^{c} u\right|$-a.e. $x \in B\left(x_{0}, \varepsilon\right)$

$$
\begin{aligned}
& \lim _{\varepsilon \rightarrow 0^{+}} \frac{\mu\left(B\left(x_{0}, \varepsilon\right)\right)}{\left|D^{c} u\right|\left(B\left(x_{0}, \varepsilon\right)\right)}=0, \\
& \lim _{\varepsilon \rightarrow 0^{+}} \frac{|D u|\left(B\left(x_{0}, \varepsilon\right)\right)}{\left|D^{c} u\right|\left(B\left(x_{0}, \varepsilon\right)\right)} \text { exists and is finite } \\
& \lim _{\varepsilon \rightarrow 0^{+}} \frac{\varepsilon^{N}}{\left|D^{c} u\right|\left(B\left(x_{0}, \varepsilon\right)\right)}=0, \\
& \lim _{\varepsilon \rightarrow 0^{+}} \frac{1}{\mathcal{L}^{N}\left(B\left(x_{0}, \varepsilon\right)\right)} \int_{B\left(x_{0}, \varepsilon\right)}\left(\left|u(x)-u\left(x_{0}\right)\right|+\left|v(x)-v\left(x_{0}\right)\right|\right) d x=0 .
\end{aligned}
$$

Moreover,

$$
A(x):=\lim _{\varepsilon \rightarrow 0^{+}} \frac{D^{c} u(B(x, \varepsilon))}{\left|D^{c} u\right|(B(x, \varepsilon))}, \quad \lim _{\varepsilon \rightarrow 0^{+}} \frac{D^{c} U(B(x, \varepsilon))}{\left|D^{c} U\right|(B(x, \varepsilon))}=: D(x)
$$

exist and they are rank-one matrices of norm 1, in particular

$$
A(x)=a_{u}(x) \otimes \nu_{u}(x),
$$

where $\left(a_{u}(x), \nu_{u}(x)\right) \in \mathbb{R}^{d} \times S^{N-1}$. By Theorem 2.2 we have

$$
\lim _{\varepsilon \rightarrow 0^{+}} \frac{1}{\left|D^{c} u\right|\left(B\left(x_{0}, \varepsilon\right)\right)} \int_{B\left(x_{0}, \varepsilon\right)} f^{\infty}\left(v\left(x_{0}\right), A(x)\right) d\left|D^{c} u\right|=f^{\infty}\left(v\left(x_{0}\right), A\left(x_{0}\right)\right) .
$$


We recall as in Step 1, that via the Global Method (cf. 114, Theorem 4.1.4]) we can obtain an integral representation for the functional $\mathcal{G}(u ; A)$ in (5.6) for every $(v, u) \in B V\left(\Omega ; \mathbb{R}^{m+d}\right)$. Moreover by Proposition 3.6. we can replace $f$ by $Q f$ in (1.11) and (5.7) holds.

Differentiating with respect to $\left|D^{c} u\right|$ at $x_{0}$ and exploiting (5.9) and (5.10) we deduce

$$
\frac{d \mathcal{F}((v, u) ; \cdot)}{d\left|D^{c} u\right|}\left(x_{0}\right) \leq h\left(x_{0}, a_{u}, \nu_{u}\right)
$$

where $\nu_{u}(x)$ agrees with the unit vector that, together with $a_{u}$, satisfies (5.12) for $\left|D^{c} u\right|$-a.e. $x \in \Omega \backslash J_{u}$, and where $h\left(x_{0}, a, \nu\right)$ is given as in [14, formula (4.1.7)], namely

$$
h\left(x_{0}, a, \nu\right):=\limsup _{k \rightarrow \infty} \limsup _{\varepsilon \rightarrow 0^{+}} \inf _{\substack{z \in W^{1,1}\left(Q_{\nu}^{(k)} ; \mathbb{R}^{d}\right) \\ z(y)=a(\nu \cdot y) \text { on } \partial Q_{\nu}^{(k)}}}\left\{\frac{1}{k^{N-1}} \int_{Q_{\nu}^{(k)}} Q f^{\infty}\left(v\left(x_{0}+\varepsilon y\right), \nabla z(y)\right) d y\right\},
$$

where $a \in \mathbb{R}^{d}, \nu \in S^{N-1}, Q_{\nu}^{(k)}:=R_{\nu}\left(\left(-\frac{k}{2}, \frac{k}{2}\right)^{N-1} \times\left(-\frac{1}{2}, \frac{1}{2}\right)\right)$, and $R_{\nu}$ is a rotation such that $R_{\nu}\left(e_{N}\right)=\nu$.

We also recall that by (iv) in Remark 3.2 $Q\left(f^{\infty}\right)=(Q f)^{\infty}=Q f^{\infty}$.

To conclude the proof it is enough to show that

$$
h\left(x_{0}, a, \nu\right) \leq Q f^{\infty}\left(v\left(x_{0}\right), a \otimes \nu\right) .
$$

By Lemma 3.10

$$
h\left(x_{0}, a, \nu\right) \leq \limsup _{k \rightarrow \infty} \inf _{\substack{z \in W^{1,1}\left(Q_{\nu}^{(k)} ; \mathbb{R}^{d}\right) \\ z(y)=a(\nu \cdot y) \text { on } \partial Q_{\nu}^{(k)}}}\left\{\limsup _{\varepsilon \rightarrow 0^{+}} \frac{1}{k^{N-1}} \int_{Q_{\nu}^{(k)}} Q f^{\infty}\left(v\left(x_{0}+\varepsilon y\right), \nabla z(y)\right) d y\right\} .
$$

In order to compute $\limsup _{\varepsilon \rightarrow 0^{+}} \frac{1}{k^{N-1}} \int_{Q_{\nu}^{(k)}} Q f^{\infty}\left(v\left(x_{0}+\varepsilon y\right), \nabla z(y)\right) d y$, we add and subtract inside the integral $Q f^{\infty}\left(v\left(x_{0}\right), \nabla z(y)\right)$. Then, as in Step 1, exploiting the fact that $x_{0}$ is a Lebesgue point for $v \in$ $S B V_{0}\left(\Omega ; \mathbb{R}^{m}\right) \cap L^{\infty}\left(\Omega ; \mathbb{R}^{m}\right)$, and that $Q f^{\infty}$ satisfies $\left(F_{3}\right)$ (see Remark 3.2 where $\left(F_{3}\right)$ has been deduced for $f^{\infty}$ and Proposition 3.1), via Lebesgue dominated convergence theorem, we can conclude that

$$
\limsup _{\varepsilon \rightarrow 0^{+}} \frac{1}{k^{N-1}} \int_{Q_{\nu}^{(k)}} Q f^{\infty}\left(\left(v\left(x_{0}+\varepsilon y\right), \nabla z(y)\right) d y\right)=\frac{1}{k^{N-1}} \int_{Q_{\nu}^{(k)}} Q f^{\infty}\left(v\left(x_{0}\right), \nabla z(y)\right) d y .
$$

Finally the quasiconvexity of $Q f^{\infty}$ (deduced via Remark 3.2 and Proposition 3.1) provides

$$
Q f^{\infty}\left(v\left(x_{0}\right), a \otimes \nu\right)=\inf _{\substack{z \in W^{1,1}\left(Q_{\nu}^{(k)} ; \mathbb{R}^{d}\right) \\ z(y)=a(\nu \cdot y) \text { on } \partial Q_{\nu}^{(k)}}}\left\{\frac{1}{k^{N-1}} \int_{Q_{\nu}^{(k)}} Q f^{\infty}\left(v\left(x_{0}\right), \nabla z(y)\right) d y\right\},
$$

which, together with (5.14) concludes the proof of the upper bound for the Cantor part when $(v, u) \in$ $\left(S B V_{0}\left(\Omega ; \mathbb{R}^{m}\right) \times B V\left(\Omega ; \mathbb{R}^{d}\right)\right) \cap L^{\infty}\left(\Omega ; \mathbb{R}^{m+d}\right)$.

Step 3. We prove the upper bound for the jump. Namely, we claim that

$$
\mathcal{F}\left(U ; J_{U}\right) \equiv \mathcal{F}\left(v, u, J_{(v, u)}\right) \leq \int_{J_{U}} K_{3}\left(v^{+}, v^{-}, u^{+}, u^{-}, \nu\right) d \mathcal{H}^{N-1}
$$

for every $U \equiv(v, u) \in\left(S B V_{0}\left(\Omega ; \mathbb{R}^{m}\right) \times B V\left(\Omega ; \mathbb{R}^{d}\right)\right) \cap L^{\infty}\left(\Omega ; \mathbb{R}^{m+d}\right)$.

The proof is divided into three parts according to the assumptions on the limit function $U$.

Case 1- $U(x):=(a, c) \chi_{E}(x)+(b, d)\left(1-\chi_{E}(x)\right)$ with $P(E, \Omega)<\infty$.

Case 2- $U(x):=\sum_{i=1}^{\infty}\left(a_{i}, c_{i}\right) \chi_{E_{i}}(x)$ where $\left\{E_{i}\right\}_{i=1}^{\infty}$ forms a partition of $\Omega$ into sets of finite perimeter and $\left(a_{i}, c_{i}\right) \in \mathbb{R}^{m} \times \mathbb{R}^{d}$. 
Case 3- $U \in\left(S B V_{0}\left(\Omega ; \mathbb{R}^{m}\right) \times B V\left(\Omega ; \mathbb{R}^{d}\right)\right) \cap L^{\infty}\left(\Omega ; \mathbb{R}^{m+d}\right)$.

Case 1 - We start by proving that for every open set $A \subset \Omega$

$$
\mathcal{F}(U ; A) \equiv \mathcal{F}(v, u ; A) \leq \int_{A} Q f(v(x), 0) d x+\int_{J_{U} \cap A} K_{3}(a, b, c, d, \nu) d \mathcal{H}^{N-1}
$$

a) Assume first that

$$
v(x):=\left\{\begin{array}{ll}
a & \text { if } x \cdot \nu>0, \\
b & \text { if } x \cdot \nu<0,
\end{array} \text { and } u(x):= \begin{cases}c & \text { if } x \cdot \nu>0 \\
d & \text { if } x \cdot \nu<0\end{cases}\right.
$$

We start with the case when $A=a+\lambda Q$ is an open cube with two faces orthogonal to $\nu$, for simplicity we also assume that $\nu=e_{N}$ and $Q_{\nu}$ will be denoted simply by $Q$. Our proof develops as in [26. Proposition 4.1 and Lemma 4.2], cf. also [12, Proposition 5.1], thus we will present just the main steps. Suppose first that $a=0$ and $\lambda=1$. By Proposition 3.4 (cf. also Remark 3.5), there exists $\left(v_{n}, u_{n}\right) \in$ $\mathcal{A}_{3}(a, b, c, d, \nu)$ such that $\left(v_{n}, u_{n}\right) \rightarrow(v, u)$ in $L^{1}\left(Q ; \mathbb{R}^{m+d}\right)$ and

$$
K_{3}(a, b, c, d, \nu)=\lim _{n \rightarrow \infty}\left(\int_{Q} Q f^{\infty}\left(v_{n}(x), \nabla u_{n}(x)\right) d x+\int_{J_{v_{n}} \cap Q} g\left(v_{n}^{+}(x), v_{n}^{-}(x), \nu_{n}(x)\right) d \mathcal{H}^{N-1}\right) .
$$

We denote by $Q^{\prime}$ the set $\left\{x \in Q: x_{N}=0\right\}$. For $k \in \mathbb{N}$ we label the elements of $(\mathbb{Z} \cap[-k, k])^{N-1} \times\{0\}$ by $\left\{a_{i}\right\}_{i=1}^{(2 k+1)^{N-1}}$ and we observe that

$$
(2 k+1) \overline{Q^{\prime}}=\bigcup_{i=1}^{(2 k+1)^{N-1}}\left(a_{i}+\overline{Q^{\prime}}\right)
$$

with

$$
\left(a_{i}+Q^{\prime}\right) \cap\left(a_{j}+Q^{\prime}\right)=\emptyset \text { for } i \neq j \text {. }
$$

We define

$$
z_{n, k}(x):= \begin{cases}a & \text { if } x_{N}>\frac{1}{2(2 k+1)} \\ v_{n}((2 k+1) x) & \text { if }\left|x_{N}\right|<\frac{1}{2(2 k+1)} \\ b & \text { if } x_{N}<-\frac{1}{2(2 k+1)}\end{cases}
$$

and

$$
w_{n, k}(x):= \begin{cases}c & \text { if } x_{N}>\frac{1}{2(2 k+1)} \\ u_{n}((2 k+1) x) & \text { if }\left|x_{N}\right|<\frac{1}{2(2 k+1)} \\ d & \text { if } x_{N}<-\frac{1}{2(2 k+1)}\end{cases}
$$

By the periodicity of the functions $v_{n}$ and $u_{n}$, it is easily seen that

$$
\lim _{n \rightarrow \infty} \lim _{k \rightarrow \infty}\left\|z_{n, k}-v\right\|_{L^{1}\left(Q ; \mathbb{R}^{m}\right)}=0, \quad \quad \lim _{n \rightarrow \infty} \lim _{k \rightarrow \infty}\left\|w_{n, k}-u\right\|_{L^{1}\left(Q ; \mathbb{R}^{d}\right)}=0 .
$$

Thus, by a standard diagonalization argument, we have

$$
\mathcal{F}(v, u ; Q) \leq \limsup _{n \rightarrow \infty} \limsup _{k \rightarrow \infty}\left(\int_{Q} Q f\left(z_{n, k}(x), \nabla w_{n, k}(x)\right) d x+\int_{Q \cap J_{z_{n}, k}} g\left(z_{n, k}^{+}(x), z_{n, k}^{-}(x), \nu_{n, k}(x)\right) d \mathcal{H}^{N-1}\right)
$$

Arguing as in [12, Proposition 5.1] for the bulk part we have

$$
\limsup _{k \rightarrow \infty} \int_{Q} Q f\left(z_{n, k}(x), \nabla w_{n, k}(x)\right) d x=\int_{Q} Q f(v(y), 0) d y+\int_{Q} Q f^{\infty}\left(v_{n}(y), \nabla u_{n}(y)\right) d y,
$$


and for the surface term

$$
\int_{Q \cap J_{z_{n, k}}} g\left(z_{n, k}^{+}(x), z_{n, k}^{-}(x), \nu_{n, k}(x)\right) d \mathcal{H}^{N-1} \leq \int_{Q \cap J_{v_{n}}} g\left(v_{n}^{+}(y), v_{n}^{-}(y), \nu_{n}(y)\right) d \mathcal{H}^{N-1}(y) .
$$

Putting together the estimates for bulk and surface terms and exploiting (5.16) we obtain that

$$
\begin{aligned}
& \mathcal{F}(v, u ; Q) \leq \limsup _{n \rightarrow \infty}\left(\int_{Q} Q f(v, 0) d x+\int_{Q} Q f^{\infty}\left(v_{n}(y), \nabla u_{n}(y)\right) d y\right. \\
& \left.+\int_{Q \cap J_{v_{n}}} g\left(v_{n}^{+}(y), v_{n}^{-}(y), \nu_{n}(y)\right) d \mathcal{H}^{N-1}\right)=\int_{Q} Q f(v(x), 0) d x+K_{3}\left(a, b, c, d, e_{N}\right) \\
& =\frac{Q f(a, 0)+Q f(b, 0)}{2}+K_{3}\left(a, b, c, d, e_{N}\right) .
\end{aligned}
$$

In order to consider sets $A=a+\lambda Q$ with $a \in \mathbb{R}^{N}$ and $\lambda>0$ we define

$$
(Q f)_{\lambda}(b, B):=Q f\left(b, \frac{B}{\lambda}\right), \quad g_{\lambda}(\xi, \zeta, \nu):=\frac{1}{\lambda} g(\xi, \zeta, \nu)
$$

and for every $E \subset \Omega$,

$$
\begin{aligned}
& \mathcal{F}_{\lambda}(v, u ; E):=\inf _{\left\{\left(v_{n}, u_{n}\right)\right\}}\left\{\liminf _{n \rightarrow \infty}\left(\int_{E}(Q f)_{\lambda}\left(v_{n}(x), \nabla u_{n}(x)\right) d x+\int_{E \cap J_{v_{n}}} g_{\lambda}\left(v_{n}^{+}(x), v_{n}^{-}(x), \nu_{n}(x)\right) d \mathcal{H}^{N-1}\right):\right. \\
& \left.\left(v_{n}, u_{n}\right) \in S B V_{0}\left(E ; \mathbb{R}^{m}\right) \times W^{1,1}\left(E ; \mathbb{R}^{d}\right),\left(v_{n}, u_{n}\right) \rightarrow(v, u) \text { in } L^{1}\left(E ; \mathbb{R}^{m+d}\right)\right\} .
\end{aligned}
$$

It is easily seen that for every $(v, u) \in L^{1}\left(\Omega ; \mathbb{R}^{m+d}\right)$, we have

$$
\mathcal{F}(v, u ; A)=\lambda^{N} \mathcal{F}_{\lambda}\left(v_{\lambda}, u_{\lambda} ; Q\right),
$$

where

$$
v_{\lambda}(x):=v\left(\frac{x-a}{\lambda}\right), u_{\lambda}(x):=u\left(\frac{x-a}{\lambda}\right) .
$$

Since $Q f_{\lambda}^{\infty}=\frac{1}{\lambda} Q f^{\infty}$, by the definition of $K_{3}$ for $f_{\lambda}$ and $g_{\lambda}$ we have that $\left(K_{3}\right)_{\lambda}(a, b, c, d, \nu)=$ $\frac{1}{\lambda} K_{3}(a, b, c, d, \nu)$.

By the definition of $u_{\lambda}$ and $v_{\lambda}$ we have that

$$
v_{\lambda}=\left\{\begin{array}{l}
a \text { if } x_{N}>0, \\
b \text { if } x_{N}<0,
\end{array} \quad u_{\lambda}=\left\{\begin{array}{l}
c \text { if } x_{N}>0 \\
d \text { if } x_{N}<0
\end{array}\right.\right.
$$

So by the previous case it results that

$$
\mathcal{F}(v, u ; A) \lambda^{N}=\mathcal{F}_{\lambda}\left(v_{\lambda}, u_{\lambda} ; Q\right) \leq \lambda^{N}\left(\frac{Q f_{\lambda}(a, 0)+Q f_{\lambda}(b, 0)}{2}+\left(K_{3}\right)_{\lambda}\left(a, b, c, d, e_{N}\right)\right) .
$$

b) Now let $U:=(v, u)$ as in $a)$ and let $A$ be any open set. The proof of this step is identical to [25, Section 5. Step 3, case 1., b)]. Indeed it is enough to apply the same strategy replacing $u$ and $K$ in [25] by $U$ and $K_{3}$ respectively herein, obtaining

$$
\mathcal{F}(v, u ; A) \leq \int_{A} Q f(v(x), 0) d x+\int_{J_{U} \cap A} K_{3}(a, b, c, d, \nu) d \mathcal{H}^{N-1}
$$


c) Now suppose that $U$ has a polygonal interface, i.e. $U=(a, c) \chi_{E}+(b, d)\left(1-\chi_{E}\right), E$ is a polyhedral set, i.e., $E$ is a bounded strongly Lipschitz domain and $\partial E=H_{1} \cup H_{2} \cup \cdots \cup H_{M}$ are closed subsets of hyperplanes of type $\left\{x \in \mathbb{R}^{N}: x \cdot \nu_{i}=\alpha_{i}\right\}$.

The details of the proof are omitted since they are very similar to [25, Section 5, Step 3, case 1, c)] . We just observe that, given an open set $A$ contained in $\Omega$, the argument relies on an inductive procedure on $I:=\left\{i \in\{1, \ldots, M\}: \mathcal{H}^{N-1}\left(H_{i} \cap A\right)>0\right\}$, starting from the case $I=0$, when $u \in W^{1,1}\left(A ; \mathbb{R}^{d}\right)$ and $v \in S B V_{0}\left(A ; \mathbb{R}^{m}\right) \cap L^{\infty}\left(A ; \mathbb{R}^{m}\right)$, for which it suffices to consider $u_{n}=u$ and $v_{n}=v$ with (5.17) reducing to

$$
\mathcal{F}(v, u ; A) \leq \int_{A} Q f(v(x), 0) d x .
$$

The case card $I=1$ was studied in part $b$ ) where $E$ is a large cube so that $J_{U} \cap \Omega$ reduces to the flat interface $\{x \in \Omega: x \cdot \nu=0\}$.

Then the induction step, which first assumes that (5.17) is true if card $I=k, k \leq M-1$ and then proves that it is still true if card $I=k$, develops exactly as in [12, Proposition 5.1, Step 2, c)], the only difference being that the slicing method used to connect the sequence across the interfaces relies on the same techniques as Lemma 3.8, referred to more general open sets than cubes (cf. also [25, Section 5 , Step 3 , case $1, \mathrm{c}])$. Thus one can conclude that

$$
\mathcal{F}(v, u ; A) \leq \int_{A} Q f(v(x), 0) d x+\int_{J_{U} \cap A} K_{3}(a, b, c, d, \nu) d \mathcal{H}^{N-1} .
$$

d) If $E$ is an arbitrary set of finite perimeter, the step develops in strong analogy with 25, Section 5, Step 3, case 1, f)]. Essentially, exploiting Proposition 3.3 (b) and the approximation via polyhedral sets with finite perimeter as in [11, Lemma 3.1], and application of Lebesgue's monotone convergence theorem gives

$$
\mathcal{F}(v, u ; A) \leq \int_{A} Q f(v(x), 0) d x+\int_{A \cap J_{U}} K_{3}(a, b, c, d, \nu) d \mathcal{H}^{N-1},
$$

This last inequality, together with Lemma 5.2 , yields

$$
\mathcal{F}\left(v, u ; J_{(v, u)}\right) \leq \int_{J_{(v, u)}} K_{3}(a, b, c, d, \nu) d \mathcal{H}^{N-1}
$$

which gives (5.15) when $U \equiv(v, u)=(a, c) \chi_{E}+(b, d)\left(1-\chi_{E}\right)$ is the characteristic function of a set of finite perimeter.

Case 2- Arguing as in [25, Section 5, Step 3, case 2], we refer to 9, Proposition 4.8, Step 1], and clearly we obtain for every $(v, u) \in B V(\Omega ; T) \times B V(\Omega ; T)$, with $T$ a finite subset of $\mathbb{R}^{d}$

$$
\mathcal{F}(v, u ; A)=\mathcal{F}\left(v, u ; A \cap J_{(v, u)}\right) \leq \int_{J_{(v, u)}} K_{3}\left(v^{+}, v^{-}, u^{+}, u^{-}, \nu_{v, u}(x)\right) d \mathcal{H}^{N-1}(x) .
$$

Case 3- For $U \equiv(v, u) \in\left(S B V_{0}\left(\Omega ; \mathbb{R}^{m}\right) \times B V\left(\Omega ; \mathbb{R}^{d}\right)\right) \cap L^{\infty}\left(\Omega ; \mathbb{R}^{m+d}\right)$, the proof develops analogously to [9, Proposition 4.8, Step 2] and we add some details for the reader's convenience.

First we observe that the jump set $J_{U} \equiv J_{(v, u)}$ can be decomposed as $\left(J_{u} \backslash J_{v}\right) \cup\left(J_{v} \backslash J_{u}\right) \cup\left(J_{u} \cap J_{v}\right)$, recalling that these sets are mutually disjoint and the tangent hyperplanes to $J_{u}$ and $J_{v}$ coincide up to a set of $\mathcal{H}^{N-1}$ - measure 0 .

Let $A \in \mathcal{A}(\Omega)$, such that $A \supset J_{U}$, we assume $U(x) \in[0,1]^{m+d}$ for a.e. $x \in A$. For every $h \in \mathbb{N}, h \geq 2$, it is possible to define a set $B_{h}:=A \backslash J_{U} \cup\left\{x \in J_{U}:\left|U^{+}(x)-U^{-}(x)\right| \leq \frac{1}{4(m+d) h}\right\}$, and define the sequence $\left\{U_{h}\right\} \equiv\left\{\left(v_{h}, u_{h}\right)\right\}$ according to [9, Proposition 4.8, Step 2]. Observe that $J_{v_{h}} \subset J_{v}$. Then, by Step 2, we have that

$$
\begin{aligned}
\mathcal{F}(v, u, ; A) \leq \liminf _{h \rightarrow \infty} \mathcal{F}\left(v_{h}, u_{h} ; A\right) & =\liminf _{h \rightarrow \infty}\left(\int_{A} Q f\left(v_{h}, 0\right) d x+\int_{A} Q f^{\infty}\left(v_{h}, \frac{d D^{c} u_{h}}{d\left|D^{c} u_{h}\right|}\right) d\left|D^{c} u_{h}\right|\right. \\
+ & \left.\int_{A \cap\left(J_{u_{h}} \cup J_{v_{h}}\right)} K_{3}\left(v_{h}^{+}, v_{h}^{-}, u_{h}^{+}, u_{h}^{-}, \nu_{v_{h}, u_{h}}\right) d \mathcal{H}^{N-1}\right) .
\end{aligned}
$$


We restrict our attention to the surface integral. Clearly,

$$
\begin{aligned}
& \int_{A \cap\left(J_{u_{h}} \cup J_{v_{h}}\right)} K_{3}\left(v_{h}^{+}, v_{h}^{-}, u_{h}^{+}, u_{h}^{-}, \nu_{v_{h}, u_{h}}\right) d \mathcal{H}^{N-1}=\int_{A \cap\left(J_{u_{h}} \cup J_{v_{h}}\right) \cap B_{h}} K_{3}\left(v_{h}^{+}, v_{h}^{-}, u_{h}^{+}, u_{h}^{-}, \nu_{v_{h}, u_{h}}\right) d \mathcal{H}^{N-1} \\
& +\int_{A \cap\left(J_{u_{h}} \cup J_{v_{h}}\right) \cap\left(A \backslash B_{h}\right)} K_{3}\left(v_{h}^{+}, v_{h}^{-}, u_{h}^{+}, u_{h}^{-}, \nu_{v_{h}, u_{h}}\right) d \mathcal{H}^{N-1} .
\end{aligned}
$$

By the decomposition of the jump set $J_{\left(v_{h}, u_{h}\right)}$, Proposition 3.3 d), the fact that $J_{v_{h}} \subset J_{v}$, the same type of estimates as in [9, page 300], entail (with the constant $C$ varying from place to place)

$$
\begin{aligned}
& \int_{A \cap\left(J_{u_{h}} \cup J_{v_{h}}\right) \cap B_{h}} K_{3}\left(v_{h}^{+}, v_{h}^{-}, u_{h}^{+}, u_{h}^{-}, \nu_{v_{h}, u_{h}}\right) d \mathcal{H}^{N-1}=\int_{A \cap\left(J_{u_{h}} \backslash J_{v_{h}}\right) \cap B_{h}} K_{3}\left(v_{h}^{+}, v_{h}^{-}, u_{h}^{+}, u_{h}^{-}, \nu_{v_{h}, u_{h}}\right) d \mathcal{H}^{N-1} \\
& +\int_{A \cap\left(J_{v_{h}} \backslash J_{u_{h}}\right) \cap B_{h}} K_{3}\left(v_{h}^{+}, v_{h}^{-}, u_{h}^{+}, u_{h}^{-}, \nu_{v_{h}, u_{h}}\right) d \mathcal{H}^{N-1}+\int_{A \cap J_{u_{h}} \cap J_{v_{h}} \cap B_{h}} K_{3}\left(v_{h}^{+}, v_{h}^{-}, u_{h}^{+}, u_{h}^{-}, \nu_{v_{h}, u_{h}}\right) d \mathcal{H}^{N-1} \\
& \leq C \int_{A \cap\left(J_{u_{h}} \backslash J_{v_{h}}\right) \cap B_{h}}\left|u_{h}^{+}-u_{h}^{-}\right| d \mathcal{H}^{N-1}+C \int_{A \cap\left(J_{v_{h}} \backslash J_{u_{h}}\right) \cap B_{h}}\left(\left|v_{h}^{+}-v_{h}^{-}\right|+1\right) d \mathcal{H}^{N-1} \\
& +C \int_{A \cap J_{u_{h}} \cap J_{v_{h}} \cap B_{h}}\left(\left|v_{h}^{+}-v_{h}^{-}\right|+\left|u_{h}^{+}-u_{h}^{-}\right|+1\right) d \mathcal{H}^{N-1} \\
& \leq 2 C(m+d)|D u|\left(A \cap B_{h}\right)+C(m+d)|D v|\left(A \cap B_{h}\right)+C \mathcal{H}^{N-1}\left(J_{v} \cap B_{h} \cap A\right),
\end{aligned}
$$

. Moreover, by Proposition 3.3 c), d) and reverse Fatou's lemma we have

$$
\int_{\left(J_{v_{h}} \cup J_{u_{h}}\right) \cap\left(A \backslash B_{h}\right)} K_{3}\left(v_{h}^{+}, v_{h}^{-}, u_{h}^{+}, u_{h}^{-}, \nu_{\left(v_{h}, u_{h}\right)}\right) d \mathcal{H}^{N-1} \leq \int_{A \cap\left(J_{v} \cup J_{u}\right)} K_{3}\left(v^{+}, v^{-}, u^{+}, u^{-}, \nu_{(v, u)}\right) d \mathcal{H}^{N-1} .
$$

Clearly, taking the limit as $h \rightarrow \infty$, from the above inequality and (5.19) we may conclude that,

$$
\begin{aligned}
& \mathcal{F}(v, u ; A) \leq \int_{A \cap\left(J_{v} \cup J_{u}\right)} K_{3}\left(v^{+}, v^{-}, u^{+}, u^{-}, \nu_{(v, u)}\right) d \mathcal{H}^{N-1} \\
& +C\left(|D u|\left(A \backslash\left(J_{v} \cup J_{u}\right)\right)+|D v|\left(A \backslash\left(J_{u} \cup J_{v}\right)\right)+\int_{A} Q f(v, 0) d x,\right.
\end{aligned}
$$

where we have exploited the fact that the Cantor term in (5.18) is 0 , from the construction of the $u_{h}$, and $\liminf _{h \rightarrow \infty} \mathcal{H}^{N-1}\left(J_{v} \cap B_{h} \cap A\right)=\mathcal{H}^{N-1}\left(J_{v} \cap\left(A \backslash\left(J_{u} \cup J_{v}\right)\right)\right)=0$. Now, since $\mathcal{F}(v, u ; \cdot)$ is a Radon measure, the above inequality holds for every Borel set $B$, in particular for the set $B=A \cap\left(J_{v} \cup J_{u}\right)$ and this gives

$$
\mathcal{F}\left(v, u ; J_{v} \cap J_{u}\right) \leq \int_{J_{v} \cap J_{u}} K_{3}\left(v^{+}, v^{-}, u^{+}, u^{-}, \nu_{(v, u)}\right) d \mathcal{H}^{N-1} .
$$

This concludes the proof of Step 2 when $(v, u) \in S B V_{0}\left(\Omega ; \mathbb{R}^{m}\right) \times B V\left(\Omega ; \mathbb{R}^{d}\right) \cap L^{\infty}\left(\Omega ; \mathbb{R}^{m+d}\right)$.

The general case $(v, u) \in S B V_{0}\left(\Omega ; \mathbb{R}^{m}\right) \times B V\left(\Omega ; \mathbb{R}^{d}\right)$ follows from (iii) in Remark 3.9. (cf. [25, Section 5, Step 4.] and [9, Theorem 4.9]).

Proof of Theorem 1.2, It follows from Theorems 4.1 and 5.1

Remark 5.3 We observe that, as it can be easily conjectured from the proof of Theorems 4.1, Step 2, and 5.1. Step 3, Case 3. i) and ii), $K_{3}$ admits the following equivalent representation:

on $J_{u} \backslash J_{v} K_{3}(a, a, c, d, \nu)=Q f^{\infty}(a,(c-d) \otimes \nu)$, where $Q f^{\infty}$ represents the recession function of the quasiconvexification of $f$ as in Remark 3.2. In fact one inequality is trivial by Definition 1.13, while the other can be obtained through Proposition 3.4, invoking the quasiconvexity and the growth properties of $Q f^{\infty}(a, \cdot)$ (cf. Remark [3.2) and analogous arguments to the ones leading to [8, formula (5.84)]. 
on $J_{v} \backslash J_{u} K_{3}(a, b, c, c, \nu)=\mathcal{R} g(a, b, \nu)$ where $\mathcal{R} g$ represents the $B V$-elliptic envelope of $g$, namely the greatest $B V$-elliptic function less than or equal to $g$, which under the assumptions $\left(G_{1}\right)-\left(G_{3}\right)$ admits the representation

$$
\mathcal{R} g(a, b, \nu)=\inf \left\{\int_{J_{w} \cap Q_{\nu}} g\left(w^{+}, w^{-}, \nu\right) d \mathcal{H}^{N-1}: w \in S B V_{0}\left(Q_{\nu} ; \mathbb{R}^{m}\right) \cap L^{\infty}\left(Q_{\nu} ; \mathbb{R}^{m}\right), w=v_{0} \text { on } \partial Q_{\nu}\right\},
$$

as in [15], [17], [13], where $v_{0}$ is defined as in (3.4). This is a consequence of (1.13) and (5.20).

We observe that the above characterizations of $K_{3}$ could be deduced directly reproducing the proof of lower bound and upper bound for Theorem 1.2, for the jump part on the sets $J_{u} \backslash J_{v}$ and $J_{v} \backslash J_{u}$, respectively.

\section{Applications}

This section is devoted to the proof of Theorem 1.1 which is very similar to that of Theorem 1.2. In particular we replace Lemma 3.8 and Proposition 3.3 by Lemma 6.1 and Proposition 6.2 respectively. Having in mind the application that we will describe in more details in Remark 6.4 we state it with more generality, but in order to prove Theorem 1.1 we will consider $m=1$ and $T=\{0,1\}$.

Let $T \subset \mathbb{R}^{m}$ be a finite set and let

$$
V: T \times \mathbb{R}^{d \times N} \rightarrow(0,+\infty) \text { and } g: T \times T \times S^{N-1} \rightarrow[0,+\infty[
$$

satisfying $\left(F_{1}\right)-\left(F_{4}\right)$ and $\left(G_{1}\right)-\left(G_{3}\right)$, respectively, and denote by $\mathcal{A}_{f r}$ the set defined in (1.8), where the range $\{0,1\}$ is replaced by $T$.

For simplicity we will consider $\nu=e_{N}$ and consequently $Q_{\nu}=Q=[0,1]^{N}$.

Lemma 6.1 Let $T \subset \mathbb{R}^{m}$ a finite set, and

$$
v_{0}(y):=\left\{\begin{array}{ll}
a & \text { if } x_{N}>0, \\
b & \text { if } x_{N}<0,
\end{array} \quad u_{0}(y):= \begin{cases}c & \text { if } x_{N}>0 \\
d & \text { if } x_{N}<0\end{cases}\right.
$$

Let $\left\{v_{n}\right\} \subset B V(\Omega ; T)$ and $\left\{u_{n}\right\} \subset W^{1,1}\left(Q ; \mathbb{R}^{d}\right)$, such that $v_{n} \rightarrow v_{0} L^{1}\left(Q ; \mathbb{R}^{m}\right)$ and $u_{n} \rightarrow u_{0}$ in $L^{1}\left(Q ; \mathbb{R}^{d}\right)$.

If $\rho$ is a mollifier, $\rho_{n}:=n^{N} \rho(n x)$, then there exists a sequence of functions $\left\{\left(\zeta_{n}, \xi_{n}\right)\right\} \in \mathcal{A}_{f r}\left(a, b, c, d, e_{N}\right)$, such that

$$
\begin{gathered}
\zeta_{n}=v_{0} \text { on } \partial Q, \zeta_{n} \rightarrow v_{0} \text { in } L^{1}\left(Q ; \mathbb{R}^{m}\right), \\
\xi_{n}=\rho_{i(n)} * u_{0} \text { on } \partial Q, \quad \xi_{n} \rightarrow u_{0} \text { in } L^{1}\left(Q ; \mathbb{R}^{d}\right)
\end{gathered}
$$

and

$$
\begin{aligned}
& \limsup _{n \rightarrow \infty}\left(\int_{Q} Q V\left(\zeta_{n}, \nabla \xi_{n}\right) d x+\int_{J_{\zeta_{n}} \cap Q} g\left(\zeta_{n}^{+}, \zeta_{n}^{-}, \nu_{\zeta_{n}}\right) d \mathcal{H}^{N-1}\right) \\
& \leq \liminf _{n \rightarrow \infty}\left(\int_{Q} Q V\left(v_{n}, \nabla u_{n}\right) d x+\int_{J_{v_{n}} \cap Q} g\left(v_{n}^{+}, v_{n}^{-}, \nu_{v_{n}}\right) d \mathcal{H}^{N-1}\right),
\end{aligned}
$$

where $Q V$ represents the quasiconvex envelope of $V$ as in (3.2).

We omit the proof since it is entirely similar to the one of Lemma 3.8. We just observe that there is no need of the first step where a truncation argument for $v$ was built, since in the present context we deal with functions with finite range.

The following result, which contains the properties satisfied by $K_{2}$ in (1.7), is analogous to Proposition 3.3 and it is stated for the reader's convenience.

Proposition 6.2 Let $V$ be as in (1.4). Let $K_{2}$ be the function introduced in (1.7). The following properties hold. 
a) $\left|K_{2}(a, b, c, d, \nu)-K_{2}\left(a^{\prime}, b^{\prime}, c^{\prime}, d^{\prime}, \nu\right)\right| \leq C\left(\left|a-a^{\prime}\right|+\left|b-b^{\prime}\right|+\left|c-c^{\prime}\right|+\left|d-d^{\prime}\right|\right)$ for every $(a, b, c, d, \nu)$, $\left(a^{\prime}, b^{\prime}, c^{\prime}, d^{\prime}, \nu\right) \in\{0,1\} \times\{0,1\} \times \mathbb{R}^{d} \times \mathbb{R}^{d} \times S^{N-1} ;$

b) $\nu \longmapsto K_{2}(a, b, c, d, \nu)$ is upper semicontinuous for every $(a, b, c, d) \in\{0,1\} \times\{0,1\} \times \mathbb{R}^{d} \times \mathbb{R}^{d}$;

c) $K_{2}$ is upper semicontinuous in $\{0,1\} \times\{0,1\} \times \mathbb{R}^{d} \times \mathbb{R}^{d} \times S^{N-1}$;

d) $K_{2}(a, b, c, d, \nu) \leq C(|a-b|+|c-d|)$ for every $\nu \in S^{N-1}$.

Proof of Theorem 1.1. The arguments develop as in Theorem 1.2, essentially replacing $f$ by $V$ in (1.4), $v$ by $\chi$, the surface integral by $|D \chi|$, and using the blow-up argument introduced in [24, thus we will present just the main differences.

Lower bound. Let $(\chi, u) \in B V(\Omega ;\{0,1\}) \times B V\left(\Omega ; \mathbb{R}^{d}\right)$. Without loss of generality we may assume that for every $\left\{\left(\chi_{n}, u_{n}\right)\right\} \subset B V(\Omega ;\{0,1\}) \times B V\left(\Omega ; \mathbb{R}^{d}\right)$ converging to $(\chi, u)$ in $L^{1}(\Omega ;\{0,1\}) \times L^{1}\left(\Omega ; \mathbb{R}^{d}\right)$, $\liminf _{n \rightarrow \infty}\left(\int_{\Omega} V\left(\chi_{n}, \nabla u_{n}\right) d x+\left|D \chi_{n}\right|(\Omega)\right)$ is indeed a limit. For every Borel set $B \subset \Omega$ define

$$
\mu_{n}(B):=\int_{B} V\left(\chi_{n}, \nabla u_{n}\right) d x+\left|D \chi_{n}\right|(B) .
$$

The sequence $\left\{\mu_{n}\right\}$ behaves as in Theorem 1.2 and its weak $*$ limit (up to a not relabelled subsequence) $\mu$ can be decomposed as in (4.2) where, as in the remainder of the proof, $J_{(v, u)}$ has been replaced by $J_{(\chi, u)}$. Moreover we emphasize that we have been considering $(\chi, u)$ as a unique field in $B V\left(\Omega ; \mathbb{R}^{1+d}\right)$ and we have been exploiting the fact that $D^{c}(\chi, u)=\left(0, D^{c} u\right)$ (cf. Remark 2.11). By Besicovitch derivation theorem we deduce (4.3).

We claim that

$$
\begin{gathered}
\mu_{a}\left(x_{0}\right) \geq Q V\left(\chi\left(x_{0}\right), \nabla u\left(x_{0}\right)\right), \text { for } \mathcal{L}^{N}-\text { a.e. } x_{0} \in \Omega, \\
\mu_{j}\left(x_{0}\right) \geq K_{2}\left(\chi^{+}\left(x_{0}\right), \chi^{-}\left(x_{0}\right), u^{+}\left(x_{0}\right), u^{-}\left(x_{0}\right), \nu_{(\chi, u)}\right), \text { for } \mathcal{H}^{N-1}-\text { a.e. } x_{0} \in J_{(\chi, u)} \cap \Omega, \\
\mu_{c}\left(x_{0}\right) \geq(Q V)^{\infty}\left(\chi\left(x_{0}\right), \frac{d D^{c} u}{d\left|D^{c} u\right|}\left(x_{0}\right)\right) \text { for }\left|D^{c} u\right|-\text { a.e. } x_{0} \in \Omega .
\end{gathered}
$$

If (6.3) - (6.5) hold then the lower bound inequality for Theorem 1.1 follows.

Step 1. Observing that by Proposition $3.1 Q V$ satisfies $\left(F_{1}\right)-\left(F_{3}\right)$, the proof of (6.3) develops as in Step 1 of Theorem [1.2, just applying [25, formula (2.10) in Theorem 2.19], to the functional $G:(\chi, u) \in$ $W^{1,1}\left(\Omega ; \mathbb{R}^{1+d}\right) \rightarrow \int_{\Omega} Q V(\chi, \nabla u) d x$.

Step 2. The proof of (6.4) is very similar to the one of (4.5). Remind that $J_{(\chi, u)}=J_{\chi} \cup J_{u}$ and $\nu_{(\chi, u)}=\nu_{\chi}$ for every $(\chi, u) \in B V(\Omega ;\{0,1\}) \times W^{1,1}\left(\Omega ; \mathbb{R}^{d}\right)$. The same arguments of Step 2. in Theorem 1.2 allow us to fix $x_{0} \in J_{(\chi, u)} \cap \Omega$ such that (4.7), (4.8), (4.9) (4.10) and (4.11) hold.

Recall that we denote $Q_{\nu\left(x_{0}\right)}$ by $Q$ and we may choose $\varepsilon>0$ such that $\mu\left(\partial\left(x_{0}+\varepsilon Q\right)\right)=0$. It results

$$
\begin{aligned}
\mu_{j}\left(x_{0}\right) & \geq \lim _{\varepsilon \rightarrow 0^{+}} \lim _{n \rightarrow \infty} \frac{1}{\varepsilon^{N-1}}\left(\int_{x_{0}+\varepsilon Q} Q V\left(\chi_{n}(x), \nabla u_{n}(x)\right) d x+\left|D \chi_{n}\right|\left(x_{0}+\varepsilon Q\right)\right) \\
& =\lim _{\varepsilon \rightarrow 0^{+}} \lim _{n \rightarrow \infty}\left(\varepsilon \int_{Q} Q V\left(\chi_{n}\left(x_{0}+\varepsilon y\right), \nabla u_{n}\left(x_{0}+\varepsilon y\right)\right) d y+\left|D \chi_{n}\left(x_{0}+\varepsilon y\right)\right|\left(Q \cap J\left(\chi_{n}, u_{n}\right)-\frac{x_{0}}{\varepsilon}\right)\right) .
\end{aligned}
$$

Define $\chi_{n, \varepsilon}, u_{n, \varepsilon}, \nu_{n, \varepsilon}$ and $\chi_{0}, u_{0}$ according to (4.12) and (4.13). Since $\left(\chi_{n}, u_{n}\right) \rightarrow(\chi, u)$ in $L^{1}\left(\Omega ; \mathbb{R}^{1+d}\right)$ we obtain (4.14) and (4.15), with $v_{n, \varepsilon}$ and $v_{0}$ replaced by $\chi_{n, \varepsilon}$ and $\chi_{0}$, respectively.

Thus

$$
\begin{aligned}
\mu_{j}\left(x_{0}\right) & \geq \lim _{\varepsilon \rightarrow 0^{+}} \lim _{n \rightarrow \infty}\left(\int_{Q} Q V^{\infty}\left(\chi_{n, \varepsilon}(y), \nabla u_{n, \varepsilon}(y)\right) d y+\left|D \chi_{n, \varepsilon}\right|(Q)\right. \\
& \left.+\int_{Q} \varepsilon Q V\left(\chi_{n, \varepsilon}(y), \frac{1}{\varepsilon} \nabla u_{n, \varepsilon}(y)\right)-Q V^{\infty}\left(\chi_{n, \varepsilon}, \nabla u_{n, \varepsilon}\right) d y\right) .
\end{aligned}
$$


By Remark $3.2(v)$ we can argue as in the estimates [25, (3.3)-(3.5)], obtaining

$$
\mu_{j}\left(x_{0}\right) \geq \liminf _{\varepsilon \rightarrow 0^{+}} \liminf _{n \rightarrow \infty}\left(\int_{Q} Q V^{\infty}\left(\chi_{n, \varepsilon}(y), \nabla u_{n, \varepsilon}(y)\right) d y+\left|D \chi_{n, \varepsilon}\right|(Q)\right) .
$$

Applying Lemma 6.1 with $Q V$ replaced by $Q V^{\infty}, T \subset \mathbb{R}^{m}$ replaced by $\{0,1\}$, the surface integral replaced by the total variation, $K_{f r}$ and $\mathcal{A}_{f r}$ replaced by $K_{2}$ and $\mathcal{A}_{2}$ respectively, and using Remark 3.2, we may find $\left\{\left(\zeta_{k}, \xi_{k}\right)\right\} \in \mathcal{A}_{2}\left(\chi^{+}\left(x_{0}\right), \chi^{-}\left(x_{0}\right), u^{+}\left(x_{0}\right), u^{-}\left(x_{0}\right), \nu\left(x_{0}\right)\right)$ such that

$$
\mu_{j}\left(x_{0}\right) \geq \lim _{k \rightarrow \infty}\left(\int_{Q} Q V^{\infty}\left(\zeta_{k}, \nabla \xi_{k}\right) d x+\left|D \zeta_{k}\right|(Q)\right) \geq K_{2}\left(\chi^{+}\left(x_{0}\right), \chi^{-}\left(x_{0}\right), u^{+}\left(x_{0}\right), u^{-}\left(x_{0}\right), \nu\left(x_{0}\right)\right) .
$$

Step 3. The proof of (6.5) follows identically as in Step 3, Theorem 4.1, namely applying [25, formula (2.12) in Theorem 2.19] to the functional $G$ introduced in Step 1 herein and this concludes the proof.

Upper Bound. The proof of the upper bound develops in three steps as the one of Theorem 5.1 Furthermore Propositions 3.6 can be readapted replacing $Q f$ by $Q V$ and the surface integral by $|D \chi|$.

Step 1. For $\mathcal{L}^{N}$ - a.e. $x_{0} \in \Omega, x_{0}$ is a Lebesgue point for $U \equiv(\chi, u)$ such that also (5.4) and (5.5) hold for $Q V$. In analogy with Theorem [5.1 Step 1- we apply for every $\chi \in B V(\Omega ;\{0,1\})$, the Global Method [14], Theorem 4.1.4] to the functional $G:(u, A) \in W^{1,1}\left(\Omega ; \mathbb{R}^{m}\right) \times \mathcal{A}(\Omega) \rightarrow \int_{\Omega} Q V(\chi, \nabla u) d x$, to obtain an integral representation for the functional (5.6) for every $(u, A) \in B V\left(\Omega ; \mathbb{R}^{m}\right) \times \mathcal{A}(\Omega)$. Moreover we can write

$$
\mathcal{F}_{\mathcal{O D}}(\chi, u ; A) \leq \mathcal{G}(u ; A)+|D \chi|(A) .
$$

Differentiating with respect to $\mathcal{L}^{N}$ we obtain $\frac{d \mathcal{F}_{\mathcal{O D}}(\chi, u ; \cdot)}{d \mathcal{L}^{N}} \leq V_{0}\left(x_{0}, \nabla u\left(x_{0}\right)\right)$, where $V_{0}$ is the correspective of $f_{0}$ in (5.8) where $Q f$ has been replaced by $Q V$. Arguing as in the last part of Theorem 5.1 Step 1, applying Lemma [3.10, we deduce that $V_{0}\left(x_{0}, \xi_{0}\right) \leq Q V\left(\chi\left(x_{0}\right), \xi_{0}\right)$ and this leads to the conclusion when $u \in B V\left(\Omega ; \mathbb{R}^{d}\right) \cap L^{\infty}\left(\Omega ; \mathbb{R}^{d}\right)$.

Step 2. The same type of arguments as in Step 1, applies to the proof of the upper bound for the Cantor part. Radon-Nikodým theorem implies (5.9) for every $U \equiv(\chi, u) \in B V(\Omega ;\{0,1\}) \times\left(B V\left(\Omega ; \mathbb{R}^{d}\right) \cap L^{\infty}\left(\Omega ; \mathbb{R}^{d}\right)\right)$, with $\left|D^{c} u\right|$ and $\sigma$ mutually singular. Moreover (5.10), (5.11), (5.12) hold, the Global Method [14, Theorem 4.1.4] applies to (5.6) and a differentiation with respect to $\left|D^{c} u\right|$ at $x_{0}$ provides $\frac{d \mathcal{F}_{\mathcal{O D}}(\chi, u ; \cdot)}{d\left|D^{c} u\right|}\left(x_{0}\right) \leq h\left(x_{0}, a_{u}, \nu_{u}\right)$, where $h(x, a, \nu)$ is given by (5.13). Remark 3.2 applied to $Q V^{\infty}$, Lemma 3.10 and the same techniques employed in the last part of Theorem 5.1.Step 2, entail

$$
h\left(x_{0}, a, \nu\right) \leq Q V^{\infty}\left(\chi\left(x_{0}\right), a \otimes \nu\right),
$$

and that concludes the proof of the Cantor part for $(\chi, u) \in B V(\Omega ;\{0,1\}) \times\left(B V\left(\Omega ; \mathbb{R}^{d}\right) \cap L^{\infty}\left(\Omega ; \mathbb{R}^{d}\right)\right)$.

Step 3. We claim that

$$
\mathcal{F}_{\mathcal{O D}}\left(U ; J_{U}\right) \leq \int_{J_{U}} K_{2}\left(\chi^{+}, \chi^{-}, u^{+}, u^{-}, \nu_{\chi, u}\right) d \mathcal{H}^{N-1},
$$

for every $(\chi, u) \in B V(\Omega ;\{0,1\}) \times\left(B V\left(\Omega ; \mathbb{R}^{d}\right) \cap L^{\infty}\left(\Omega ; \mathbb{R}^{d}\right)\right)$. The proof of (6.6) is divided in three parts, according to the assumptions on the limit functions $u$. Namely,

Case 1. $U(x):=(1, c) \chi_{E}(x)+(0, d)\left(1-\chi_{E}(x)\right)$, with $P(E, \Omega)<+\infty$,

Case 2. $u(x)=\sum_{i=1}^{\infty} c_{i} \chi_{E_{i}}(x)$, where $\left\{E_{i}\right\}_{i=1}^{\infty}$ forms a partition of $\Omega$ into sets of finite perimeter and $c_{i} \in \mathbb{R}^{d}$, Case 3. $u(x) \in B V\left(\Omega ; \mathbb{R}^{d}\right) \cap L^{\infty}\left(\Omega ; \mathbb{R}^{d}\right)$.

For what concerns Case 1, we consider first the unit open cube $Q \subset \mathbb{R}^{N}$, and make the same assumptions on the target function $U$ as in Theorem [5.1 Step 3, Case 1. Then we can invoke an argument analogous to Proposition 3.4, without invoking any truncation arguments as those in Remark 3.5. This guarantees that there exists $\left(\chi_{n}, u_{n}\right) \in \mathcal{A}_{2}\left(1,0, c, d, e_{N}\right)$ such that $\left(\chi_{n}, u_{n}\right) \rightarrow(\chi, u)$ in $L^{1}\left(Q ; \mathbb{R}^{1+d}\right)$ and

$$
K_{2}\left(1,0, c, d, e_{N}\right)=\lim _{n \rightarrow \infty}\left(\int_{Q} Q V^{\infty}\left(\chi_{n}(x), \nabla u_{n}(x)\right) d x+\left|D \chi_{n}\right|(Q)\right) .
$$


Then the proof develops exactly as Theorem [5.1] just taking into account that the sequence $z_{n, k}$ therein is built replacing $a, b$ and $v_{n}$ by 1,0 and $\chi_{n}$ respectively, thus leading to

$$
\mathcal{F}_{O D}(\chi, u ; Q) \leq \frac{Q V(1,0)+Q V(0,0)}{2}+K_{2}\left(1,0, c, d, e_{N}\right) .
$$

For what concerns a more general set $A$ than $Q$, like in Theorem 5.15tep 3, Case 1, we achieve the following representation

$$
\mathcal{F}_{\mathcal{O D}}(\chi, u ; A) \leq \int_{A} Q V(\chi(x), 0) d x+\int_{J_{U}} K_{2}(1,0, c, d, \nu) d \mathcal{H}^{N-1} .
$$

Then the strategy follows b), c), d) in Theorem 5.1 Step 3, Case 1, hence we obtain

$$
\mathcal{F}_{\mathcal{O D}}\left(\chi, u ; J_{\chi, u}\right) \leq \int_{J_{\chi, u}} K_{2}(1,0, c, d, \nu) d \mathcal{H}^{N-1} .
$$

Case 2. and Case 3. By the properties of $K_{2}$ in Proposition 6.2, the proof develops as in [9, Proposition 4.8, Case 2 and Case 3]. This concludes the proof of the upper bound when $(\chi, u) \in B V(\Omega ;\{0,1\}) \times\left(B V\left(\Omega ; \mathbb{R}^{d}\right) \cap\right.$ $\left.L^{\infty}\left(\Omega ; \mathbb{R}^{d}\right)\right)$.

The general case, since $\chi \in B V(\Omega ;\{0,1\})$ and can be fixed, is identical to [25, Section 5, Step 4.], where the truncation procedures involves just $u$.

Putting together Lower bound and Upper bound we achieve the desired result.

Remark 6.3 We observe that, as in Remark 5.3, $K_{2}$ admits the following equivalent representation:

i) on $J_{u} \backslash J_{\chi}, K_{2}(a, a, c, d, \nu)=Q V^{\infty}(a,(c-d) \otimes \nu)$, with $Q V^{\infty}$ as in (1.6).

ii) on $J_{\chi} \backslash J_{u}, K_{2}(a, b, c, c, \nu)=|(a-b) \otimes \nu|$, i.e. $\int_{J_{\chi}} K_{2}\left(\chi^{+}, \chi^{-}, u^{+}, u^{+}, \nu\right) d \mathcal{H}^{N-1}=|D \chi|(\Omega)$.

iii) Note that $K_{2}(a, b, c, d, \nu) \geq \inf \left\{\int_{Q_{\nu}}\left(Q V^{\infty}(w(x), \nabla u(x))+|\nabla w(x)|\right) d x:(w, u) \in \mathcal{A}(a, b, c, d, \nu)\right\}$, where this latter density is the density $K(a, b, c, d, \nu)$ first introduced in [25] (cf. also [8, formula (5.83)]) and

$$
\begin{aligned}
& \mathcal{A}(a, b, c, d, \nu):=\left\{(w, u) \in W^{1,1}\left(Q_{\nu} ; \mathbb{R}^{1+d}\right):(w(y), u(y))=(a, c) \text { if } y \cdot \nu=\frac{1}{2}\right. \\
& \left.(w(y), u(y))=(b, d) \text { if } y \cdot \nu=-\frac{1}{2},(w, u) \text { are } 1-\text { periodic in } \nu_{1}, \ldots, \nu_{N-1} \text { directions }\right\} .
\end{aligned}
$$

On the other hand, if $W_{i}, i=1,2$ in (1.1) are proportional (as in the model presented in [6]), i.e. $W_{2}=$ $\alpha W_{1}, \alpha>1$, taking $V$ as in (1.4), since for every $q \in[0,1] Q V^{\infty}(q, z)=q Q W_{1}^{\infty}(z)+\alpha(1-q) Q W_{1}^{\infty}(z)$, then we claim that $K_{2}$ is equal to $K$ of [25]. Indeed, without loss of generality, assuming $W_{1}$, quasiconvex and positively 1-homogeneous, it is enough to observe that for every $(w, u) \in \mathcal{A}(a, b, c, d, \nu)$,

$$
K(1,0, c, d, \nu) \geq \int_{Q_{v}}\left(w(x) W_{1}(\nabla u(x))+\alpha(1-w(x)) W_{1}(\nabla u(x))+|\nabla w(x)|\right) d x \geq \int_{Q_{\nu}}\left(W_{1}(\nabla u(x))+1\right) d x,
$$

where it has been used the fact that $\alpha+(1-\alpha) w \geq 1$ and

$$
\int_{Q_{\nu}}|\nabla w| d x \geq\left|\int_{Q_{\nu}} \nabla w\right| d x=\left|\int_{\partial Q_{\nu}} w \otimes \nu(x) d \mathcal{H}^{N-1}\right|=1 .
$$

Taking a sequence of characteristic functions $\left\{\chi_{\varepsilon}\right\}$, admissible for $\mathcal{A}_{2}(1,0, c, d, \nu)$ in (1.8), such that their value is 1 in a strip of the cube orthogonal to $\nu$ and of thickness $1-\varepsilon$, then, it results

$$
\begin{aligned}
& \int_{Q_{\nu}} W_{1}(\nabla u(x)) d x+1=\lim _{\varepsilon \rightarrow 0^{+}} \int_{Q_{\nu}}\left(\chi_{\varepsilon} W_{1}(\nabla u(x))+\alpha\left(1-\chi_{\varepsilon}\right) W_{1}(\nabla u(x)) d x+\left|D \chi_{\varepsilon}\right|\left(Q_{\nu}\right)\right. \\
& \geq K_{2}(1,0, c, d, \nu),
\end{aligned}
$$

and this proves our claim. Observe also that if $\alpha \in(0,1)$, then the result remains true, it is enough to express $W_{1}$ in terms of $W_{2}$. 
As emphasized in [6, Remark 2.4] one can consider mixtures of more than two conductive materials, hence we observe that Theorem 1.1 can be extended with minor changes to these models leading to formula (6.9) in the remark below.

Remark 6.4 Let $T$ be a finite subset of $\mathbb{R}^{m}$, Theorem 1.1 applies also to energies of the type $F_{f r}: L^{1}(\Omega ; T) \times$ $L^{1}\left(\Omega ; \mathbb{R}^{d}\right) \times \mathcal{A}(\Omega) \rightarrow[0,+\infty]$ defined by

$$
F_{f r}(v, u ; A):= \begin{cases}\int_{A} V(v, \nabla u) d x+\int_{J_{v} \cap A} g\left(v^{+}, v^{-}, \nu_{v}\right) d \mathcal{H}^{N-1} & \text { in } B V(A ; T) \times W^{1,1}\left(A ; \mathbb{R}^{d}\right), \\ +\infty & \text { otherwise. }\end{cases}
$$

Indeed, consider the relaxed localized energy of (6.7) given by

$$
\begin{gathered}
\mathcal{F}_{f r}(v, u ; A):=\inf \left\{\liminf _{n \rightarrow \infty} \int_{A} V\left(v_{n}, \nabla u_{n}\right) d x+\int_{J_{v_{n}} \cap A} g\left(v_{n}^{+}, v_{n}^{-}, \nu_{v_{n}}\right) d \mathcal{H}^{N-1}:\right. \\
\left.\left\{\left(v_{n}, u_{n}\right)\right\} \subset B V(A ; T) \times W^{1,1}\left(A ; \mathbb{R}^{d}\right),\left(v_{n}, u_{n}\right) \rightarrow(v, u) \text { in } L^{1}(A ; T) \times L^{1}\left(A ; \mathbb{R}^{d}\right)\right\},
\end{gathered}
$$

with $V$ and $g$ as in (6.1) satisfying $\left(F_{1}\right)-\left(F_{4}\right)$ and $\left(G_{1}\right)-\left(G_{3}\right)$, respectively.

Moreover define $\bar{F}_{f r}: B V(A ; T) \times B V\left(A ; \mathbb{R}^{d}\right) \times \mathcal{A}(\Omega) \rightarrow[0,+\infty]$ as

$\bar{F}_{f r}(v, u ; A):=\int_{A} Q V(v, \nabla u) d x+\int_{A} Q V^{\infty}\left(v, \frac{d D^{c} u}{d\left|D^{c} u\right|}\right) d\left|D^{c} u\right|+\int_{J_{(v, u)} \cap A} K_{f r}\left(v^{+}, v^{-}, u^{+}, u^{-}, \nu\right) d \mathcal{H}^{N-1}$

where $Q V$ is the quasiconvex envelope of $V$ given in (3.2), $Q V^{\infty}$ is the recession function of $Q V$, introduced in (1.6), and

$$
K_{f r}(a, b, c, d, \nu):=\inf \left\{\int_{Q_{\nu}} Q V^{\infty}(v, \nabla u(x)) d x+\int_{Q_{\nu}} g\left(v^{+}, v^{-}, \nu_{v}\right) d \mathcal{H}^{N-1}:(v, u) \in \mathcal{A}_{f r}(a, b, c, d, \nu)\right\},
$$

where $\mathcal{A}_{f r}$ is the set defined in (1.8), with $\{0,1\}$ replaced by the finite set $T \subset \mathbb{R}^{m}$. Thus, we are lead to the following representation: for every $(v, u) \in L^{1}(\Omega ; T) \times L^{1}\left(\Omega ; \mathbb{R}^{d}\right)$

$$
\mathcal{F}_{f r}(v, u ; A)= \begin{cases}\bar{F}_{f r}(v, u ; A) & \text { if }(v, u) \in B V(A ; T) \times B V\left(A ; \mathbb{R}^{d}\right) \\ +\infty & \text { otherwise. }\end{cases}
$$

Remark 6.5 In general we cannot expect $K_{3}=K_{\text {fr }}$ since in (6.8), the function $g$ is defined in $T \times T \times S^{N-1}$, with $T \subset \mathbb{R}^{d}$ and card(T) finite, while in (1.13), $g$ is defined in $\mathbb{R}^{d} \times \mathbb{R}^{d} \times S^{N-1}$. In particular we recall that in $J_{v} \backslash J_{u}, K_{3}$ coincides with $\mathcal{R} g$, the $S B V$-elliptic envelope of $g$ as in [13], while $K_{f r}$ in (6.8) is given by the BV-elliptic envelope introduced by Ambrosio and Braides, cf. [8, Definition 5.13]. Analogously, it is easily seen that $K_{2}$ coincides with $|D \chi|$ in $J_{\chi} \backslash J_{u}$.

\section{Acknowledgements}

This paper has been written during various visits of the authors at Departamento de Matemática da Universidade de Évora and at Dipartimento di Ingegneria Industriale dell' Universitá di Salerno, whose kind hospitality and support have been gratefully acknowledged.

The authors are indebted to Irene Fonseca for having suggested this problem and for the many discussions on the subject.

The work of both authors was partially supported by Fundação para a Ciência e a Tecnologia (Portuguese Foundation for Science and Technology) through CIMA-UE, UTA-CMU/MAT/0005/2009 and through GNAMPA project 2013 'Funzionali supremali: esistenza di minimi e condizioni di semicontinuitá nel caso vettoriale'. 


\section{References}

[1] Acerbi E. \& Fusco N. Semicontinuity problems in the calculus of variations, Arch. Rational Mech. Anal., 86 (1984), 125-145.

[2] Ambrosio L. A compactness theorem for a special class of functions of bounded variation, Boll. Un. Mat. Ital. B, (7), 3, (1989), 857-881.

[3] Ambrosio L. Existence theory for a new class of variational problems, Arch. Ration. Mech. Anal. 111, (1990), 291-322.

[4] Ambrosio L. A new proof of the SBV compactness theorem, Calc. Var., 3, (1995), 127-137.

[5] Ambrosio L. \& Braides A. Functionals defined on partitions in sets of finite perimeter. I: Integral representation and Gamma-convergence, J. Math. Pures Appl., IX. 69, No.3, (1990), 285-306.

[6] Ambrosio L. \& Buttazzo G. An optimal design problem with perimeter penalization, Calc. Var. Partial Differ. Equ., 1, No.1, (1993), 55-69.

[7] Ambrosio L. \& Dal Maso G. On the relaxation in $B V\left(\Omega ; \mathbb{R}^{m}\right)$ of quasi-convex integrals, Journal of Functional Analysis, 109, (1992), 76-97.

[8] Ambrosio L., Fusco N.\& Pallara D. Functions of bounded variation and free discontinuity problems Oxford Mathematical Monographs. Oxford: Clarendon Press. xviii, 434 p., (2000).

[9] Ambrosio L., Mortola S. \& Tortorelli V. M. Functionals with linear growth defined on vector valued BV functions, J. Math. Pures et Appl. 70 (1991), 269- 323.

[10] Babadjian J.-F., Zappale E.\& Zorgati H. Dimensional reduction for energies with linear growth involving the bending moment, J. Math. Pures Appl. 90, (2008), 530-549.

[11] BALdo S. Minimal interface criterion for phase transitions in mixtures of Cahn-Hilliard fluids, Annal. I. H. P., 7, (1990), 67-90.

[12] Barroso A. C., Bouchitté G., Buttazzo G. \& Fonseca I. Relaxation of bulk and interfacial energies. Arch. Ration. Mech. Anal. 135, No. 2, (1996), 107-173.

[13] Bouchitté G., Fonseca I., Leoni G. \& Mascarenhas L. A Global Method for Relaxation in $W^{1, p}$ and $S B V_{p}$, Arch. Ration. Mech. Anal, 165, (2002), 187-242.

[14] Bouchitté G., Fonseca I. \& Mascarenhas L., A Global Method for Relaxation, Arch. Ration. Mech. Anal, 144, (1998), 51-98.

[15] Braides A., Defranceschi A. \& Vitali E. Homogenization of Free Discontinuity Problems, Arch. Rational Mech. Anal. 135, (1996), 297-356.

[16] Carita G. \& Zappale E. 3D-2D dimensional reduction for a nonlinear optimal design problem with perimeter penalization, Comptes Rendus Mathematique, 350, issues 23-24, (2012), 1011-1016.

[17] Choksi R., \& Fonseca I. Bulk and interfacial energy densities for structured deformations of continua, Arch. Ration. Mech. Anal., 138 (1997) 37-103.

[18] Congedo G. \& Tamanini I. On the existence of solutions to a problem in multidimensional segmentation, Annales de l' Institut Henri Poincaré. Analyse nonlinéaire, 8, n. 21, (1991), 175-195.

[19] Dacorogna B. Direct Methods in the Calculus of Variations, 2nd ed., Applied Mathematical Sciences 78, Springer Verlag, Berlin, 2008.

[20] Dal Maso G., Fonseca I., \& Leoni G. Nonlocal character of the reduced theory of thin films with higher order perturbations, Adv. Calc. Var., 3 n. 3, (2010), 287-319. 
[21] Evans L. C. \& Gariepy R. F. Measure Theory and fine properties of functions, CRC Press, 1992.

[22] Federer H. Geometric Measure Theory, Springer Verlag, Berlin, (1969).

[23] Fonseca I.\& Leoni G. Modern Methods in the Calculus of Variations: $L^{p}$ Spaces, Springer Verlag, 2007.

[24] Fonseca I. \& MÜLler S. Quasi-convex integrands and lower semicontinuity in $L^{1}$, SIAM J. Math. Anal., 23 (1992) 1081-1098.

[25] Fonseca I. \& MülleR S. Relaxation of quasiconvex functionals in $B V\left(\Omega, \mathbb{R}^{d}\right)$ for integrands $f(x, u, \nabla u)$, Arch. Rat. Mech. Anal., 123 (1993) 1-49.

[26] Fonseca I. \& Ribka P. Relaxation of multiple integrals in the space $B V\left(\Omega ; \mathbb{R}^{d}\right)$, Proc. Roy. Soc. Edinburgh Sect. A, 121, (1992), 321-348.

[27] Giusti E. Minimal surfaces and functions of bounded variation. Monographs in Mathematics, 80. Birkhäuser Verlag, Basel, 1984.

[28] Kohn R. V. \& Strang G. Optimal design and relaxation of variational problems, I. Comm. Pure and Appl. Math. 39, 1, (1986), 113-137.

[29] Kohn R. V. \& Strang G. Optimal design and relaxation of variational problems, II. Comm. Pure and Appl. Math. 39, 2, (1986), 139-182.

[30] Kohn R. V. \& Strang G. Optimal design and relaxation of variational problems, III. Comm. Pure and Appl. Math. 39, 3, (1986), 353-377.

[31] Ribeiro A. M. \& Zappale E. Relaxation of Certain Integral Functionals Depending on Strain and Chemical composition, Chin. Ann. of Math., Ser. B, 34(B), (4), (2013), 491-514.

[32] Ziemer W. P.Weakly differentiable functions. Sobolev Spaces and Functions of Bounded Variation, Graduate Texts in Mathematics, 120, Berlin etc.: Springer-Verlag. xvi, 308 p. 